\title{
Cloud property trends in hot and ultra-hot giant gas planets (WASP-43b, WASP-103b, WASP-121b, HAT-P-7b, and WASP-18b)
}

\author{
Ch. Helling ${ }^{1,2,3}$, D. Lewis ${ }^{1,2}$, D. Samra ${ }^{1,2}$, L. Carone ${ }^{4}$, V. Graham ${ }^{6}$, O. Herbort ${ }^{1,2,5}$, K. L. Chubb ${ }^{3}$, M. Min ${ }^{3}$, \\ R. Waters ${ }^{3,7}$, V. Parmentier ${ }^{8}$, and N. Mayne ${ }^{9}$ \\ ${ }^{1}$ Centre for Exoplanet Science, University of St Andrews, North Haugh, St Andrews KY16 9SS, UK \\ e-mail: christiane.helling@st-and.ac.uk \\ 2 SUPA, School of Physics \& Astronomy, University of St Andrews, North Haugh, St Andrews KY16 9SS, UK \\ ${ }^{3}$ SRON Netherlands Institute for Space Research, Sorbonnelaan 2, 3584 CA Utrecht, The Netherlands \\ ${ }^{4}$ Max Planck Institute for Astronomy, Königstuhl 17, 69117 Heidelberg, Germany \\ ${ }^{5}$ School of Earth \& Environmental Studies, University of St Andrews, Irvine Building, St Andrews KY16 9AL, UK \\ ${ }^{6}$ School of Physics and Astronomy, University of Glasgow, University Avenue, Glasgow G12 8QQ, UK \\ ${ }^{7}$ Institute for Mathematics, Astrophysics \& Particle Physics, Department of Astrophysics, Radboud University, PO Box 9010, MC 62, \\ 6500 GL Nijmegen, The Netherlands \\ ${ }^{8}$ Department of Physics, University of Oxford, Parks Rd, Oxford OX1 3PU, UK \\ ${ }^{9}$ Physics and Astronomy, College of Engineering, Mathematics and Physical Sciences, University of Exeter EX4 4QL, UK
}

Received 14 November 2020 / Accepted 19 February 2021

\begin{abstract}
Context. Ultra-hot Jupiters are the hottest exoplanets that have been discovered so far. Observations begin to provide insight into the composition of their extended atmospheres and their chemical day/night asymmetries. Both are strongly affected by cloud formation. Aims. We explore trends in cloud properties for a sample of five giant gas planets: the hot gas giant WASP-43b and the four ultra-hot Jupiters (UHJs) WASP-18b, HAT-P-7b, WASP-103b, and WASP-121b. This provides a reference frame for cloud properties for the JWST targets WASP-43b and WASP-121b. We further explore chemically inert tracers to observe geometrical asymmetries of UHJs and if the location of the inner boundary of a 3D global circulation model (3D GCM) matters for the clouds that form.

Methods. A homogeneous set of 3D GCM results was used as input for a kinetic cloud formation code to evaluate the cloud opacity and gas parameters such as $\mathrm{C} / \mathrm{O}$, mean molecular weight, and degree of ionisation. We cast our results in terms of integrated quantities to enable a global comparison between the sample planets.

Results. The large day/night temperature differences of UHJs cause the following chemical asymmetries: cloud-free days but cloudy nights, atomic versus molecular gases and their different mean molecular weights, deep thermal ionospheres versus low-ionised atmospheres, and undepleted versus enhanced C/O. WASP-18b, as the heaviest planet in the sample, has the lowest global C/O.

Conclusions. The global climate may be considered as similar amongst UHJs, but different to that of hot gas giants. The local weather, however, is individual for each planet since the local thermodynamic conditions, and hence the local cloud and gas properties, differ. The morning and the evening terminator of UHJs will carry signatures of their strong chemical asymmetry such that ingress and egress asymmetries can be expected. An increased C/O ratio is a clear sign of cloud formation, making cloud modelling a necessity when utilising $\mathrm{C} / \mathrm{O}$ (or other mineral ratios) as a tracer for planet formation. The changing geometrical extension of the atmosphere from the day to the nightside may be probed through chemically inert species such as helium. Ultra-hot Jupiters are likely to develop deep atmospheric ionospheres which may impact the atmosphere dynamics through magneto-hydrodynamic processes.
\end{abstract}

Key words. planets and satellites: atmospheres - planets and satellites: gaseous planets - astrochemistry - solid state: refractory

\section{Introduction}

Ultra-hot Jupiters provide the unique opportunity to study a wide range of atmospheric regimes in one object due to their strong day-night temperature difference of $>2000 \mathrm{~K}$, resulting from the close proximity to their host stars in combination with an inefficient heat redistribution from the day- to the nightside (Perez-Becker \& Showman 2013). For one of the hottest known ultra-hot Jupiters, KELT-9b, several indicators for a very hightemperature dayside have been found: Ca II (Turner et al. 2020) as well as $\mathrm{Fe}$ and $\mathrm{Fe}^{+}$(Hoeijmakers et al. 2018) at the limb, in addition to an extended hydrogen-envelope (Yan \& Henning 2018) prompting investigations into the atmospheric mass loss of KELT-9b (e.g. Wyttenbach et al. 2020). Fossati et al. (2020) discuss the differences in the $\mathrm{H} \alpha$ and $\mathrm{H} \beta$ lines as observed by different groups with different instruments and different reduction pipelines. Employing non-LTE radiative transfer, a temperature of $10^{4} \ldots 1.1 \times 10^{4} \mathrm{~K}$ is retrieved to be consistent with the PEPSI transmission spectra. The first CHEOPS science paper by Lendl et al. (2020) presents WASP-189b with an global dayside temperature of $\approx 3400 \mathrm{~K}$ for a non-reflective atmosphere with inefficient heat redistribution. Also WASP-121b shows less extreme temperatures than KELT-9b such that the presence of neutral atoms such as $\mathrm{Mg}, \mathrm{Na}, \mathrm{Ca}, \mathrm{Cr}, \mathrm{Fe}$, and also $\mathrm{Ni}$ and $\mathrm{V}$ have been inferred from observations of the terminator regions (Hoeijmakers et al. 2020). However, Hoeijmakers et al. (2020) did not detect Ti and $\mathrm{TiO}$ on WASP-121b which may be an indication for cloud-depleted $\mathrm{Ti}$ abundances at the cold evening terminator similar to WASP-18b or HAT-P-7b (Helling et al. 2019a,b). Gibson et al. (2020) detect Fe I in WASP-121b applying VLT/UVES transit observations. Partial coverage of WASP-121b by aerosols was suggest along the terminator for WASP-121b by 
Table 1. Physical parameters of the exoplanets, their host star and of the orbit.

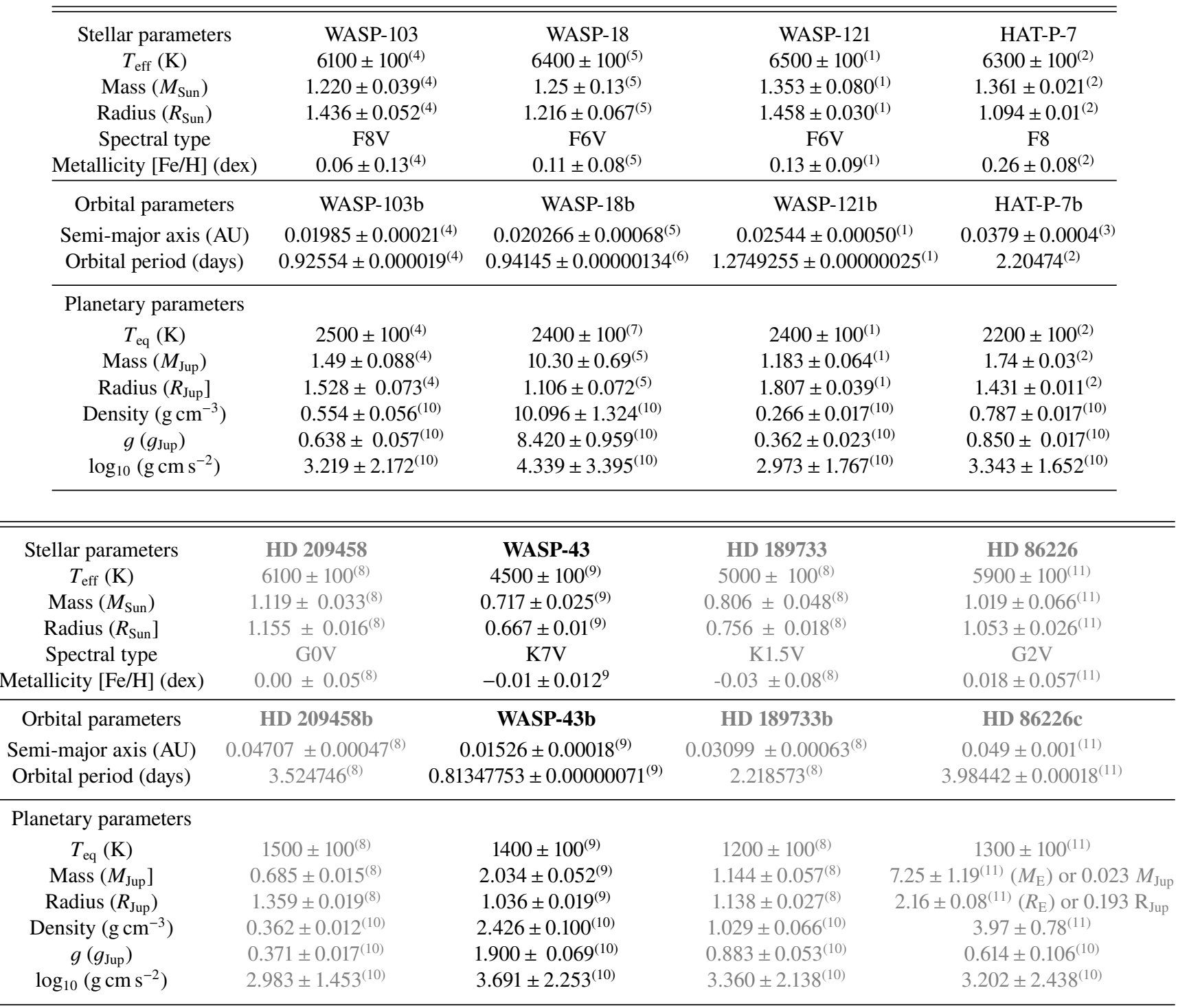

Notes. The equilibrium temperature given in the literature have been rounded to the next $100 \mathrm{~K}$ as it in unrealistic to expect that it can be determined to a precision of $1 \mathrm{~K}$ or $10 \mathrm{~K}$. Top: ultra-hot Jupiters. Bottom: hot giant gas planets and HD 86226c, a close-in sub-Neptune. Greyed planets are used for comparison only, black coloured planets are composing the gas giant sample studied here.

References: ${ }^{(1)}$ Delrez et al. (2016), ${ }^{(2)}$ Van Eylen et al. (2012), ${ }^{(3)}$ Pál (2009), ${ }^{(4)}$ Gillon et al. (2014), ${ }^{(5)}$ Hellier et al. (2009), ${ }^{(6)}$ Pearson (2019), ${ }^{(7)}$ Sheppard et al. (2017), ${ }^{(8)}$ Torres et al. $(2008),{ }^{(9)}$ Gillon et al. $(2012),{ }^{(10)}$ this work (calculated using the referenced data), ${ }^{(11)}$ Teske et al. $(2020)$.

Kempton et al. (2017) based on phase equilibrium considerations. Ehrenreich et al. (2020) present ESPRESSO/VLT observations of the ultra-hot Jupiter WASP-78b showing a day/night terminator asymmetry as suggested for WASP-18b or HAT-P-7b. Neutral iron has been suggested to be present on the hot morning terminator but not on the colder evening terminator, leading the authors to claim that iron rain should form on the nightside of WASP-78b. Based on detailed cloud modelling, Helling et al. (2019a,b) show that the dayside of the ultra-hot Jupiters WASP$18 \mathrm{~b}$ and HAT-P7b are likely to be cloud free while the nightside will be covered in clouds. In comparison, hot giant gas planets, such as HD 189733b, HD 209458b and WASP-43b, have a global day/night cloud coverage over a large pressure range (Lee et al. 2015a, 2016; Lines et al. 2018; Helling et al. 2020a) due to their smaller day-night temperature differences.

The theoretical modelling of exoplanet atmospheres has reached a level that may allow to study a set of ultra-hot Jupiters and hot giant gas planets with respect to their potential trends in cloud properties. In this paper, we consider the ultra-hot Jupiters WASP-103b, WASP-121b, HAT-P-7b, and WASP-18b, and the hot giant gas planet WASP-43b. WASP-43b and WASP$18 \mathrm{~b}$ are ERS JWST targets (program 1366). WASP-43b (program 1224), and WASP-121b (program 1201) are GTO JWST targets ${ }^{1}$. The ultra-hot Jupiters in our sample have a global temperature of $T_{\text {eq }}>2000 \mathrm{~K}$ in comparison to the hot giant gas planet $T_{\text {eq }}<1500 \mathrm{~K}$. Table 1 shows that this distinction holds also when including HD 189733b and HD 209458b. Figure 1 demonstrates that these sub-classes of giant gas planets separate well in the $g_{\mathrm{P}} / T_{\text {eq }}$ and the $T_{\text {eff }} / T_{\text {eq }}$ diagrams. As a comparison, we add the close-in sub-Neptune HD 86226c (Teske et al. 2020), which has a global temperature similar to those of hot gas giants, and

\footnotetext{
1 https://www.stsci.edu/jwst/observing-programs/ program-information
} 
Ch. Helling et al.: Trending clouds in hot giant gas planets and ultra-hot Jupiters
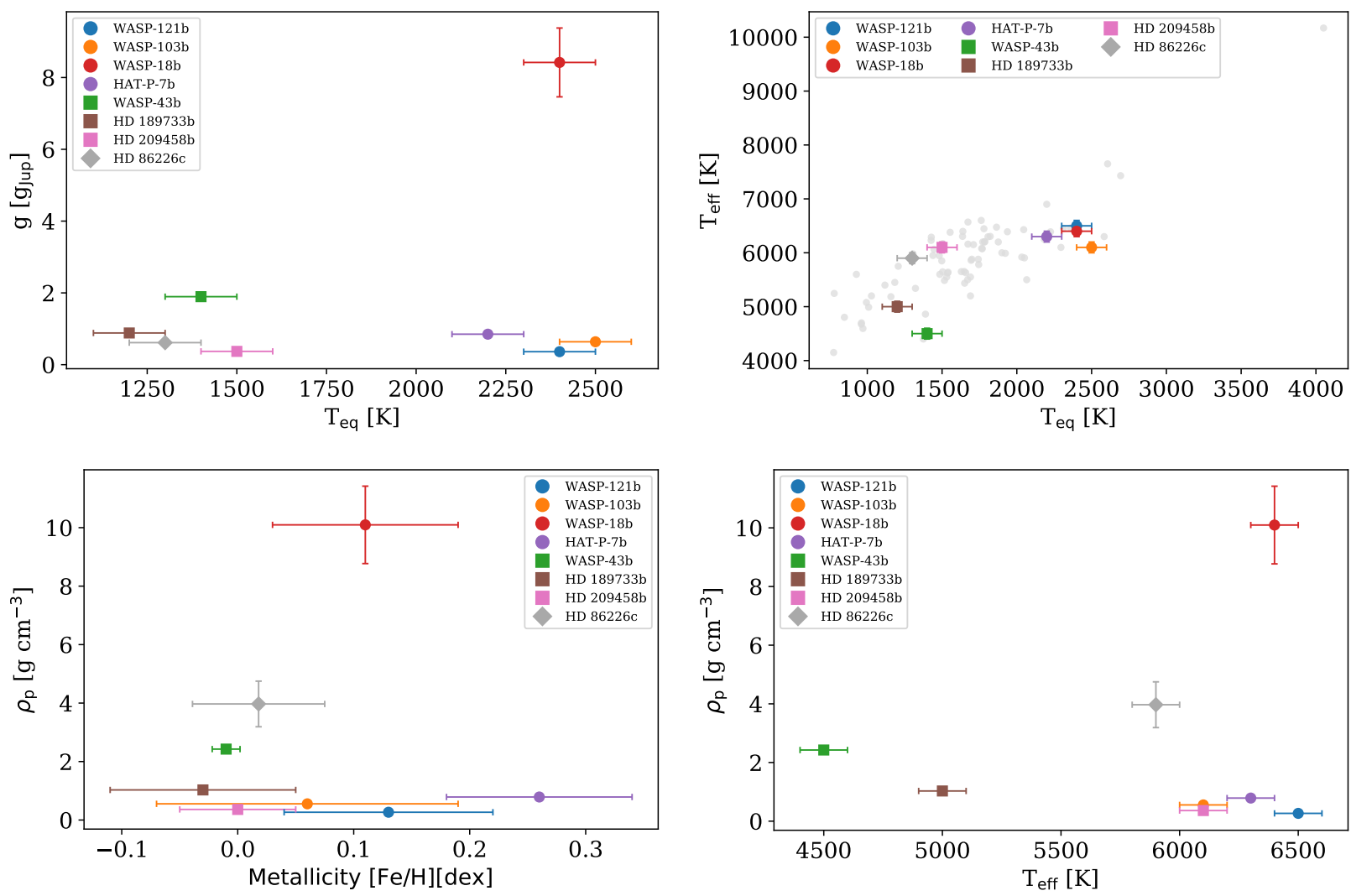

Fig. 1. Relationship between selected system properties for hot giant Jupiters (WASP-43b, HD 189733b, HD 209458b) and ultra-hot Jupiters (HAT-P-7b, WASP-18b, WASP-103b, WASP-121b). The two classes of giant gas planets are clearly separated with respect to their global planetary temperature, $T_{\text {eq }}(\mathrm{K})$ and the planets' surface gravity, $g\left(g_{\mathrm{Jup}}\right)$. There is not as clear a separation between the hot giant gas planets and ultra-hot Jupiters regarding their host star's effective temperature, $T_{\text {eff }}(\mathrm{K})$, bulk planetary density $\rho_{\mathrm{p}}$, and stellar metallicity $[\mathrm{Fe} / \mathrm{H}]$. The ultra-hot Jupiters are shown by circle markers, the giant-gas planets by the square markers and a sub-Neptune (HD 86226c) by the diamond marker. We also include the sample of hot and the ultra-hot Jupiters from Table 1 in Baxter et al. (2020) in the $T_{\text {eff }}$ versus $T_{\text {eq }}$ plot (top right) as smaller light grey points.

falls into the giant gas planet $g_{\mathrm{P}} / T_{\text {eq }}$ corner of Fig. 1 (top left). Crossfield et al. (2020) show that the IRAC2 $(4.5 \mu \mathrm{m})$ irradiation temperature of the hot Neptune LTT $9779 \mathrm{~b}$ is roughly comparable to that of HAT-P-7b, and generally consistent with that of giant gas planets.

For our comparison study, we use results from 3D GCMs as input for our kinetic cloud formation code in order to study differences and similarities of the cloud coverage of these planets. The atmosphere dynamics enters our simulation only indirectly due to its effect on the local thermodynamic properties. Starting from the global temperature/pressure/velocity structure, cloud properties such as cloud particle formation rate, mean particle size, material compositions, the dust-to-gas ratio are investigated, and also characteristic gas-phase properties such as $\mathrm{C} / \mathrm{O}$, mean molecular weight and degree of ionisation which all provide insight into various processes beyond cloud formation. $\mathrm{C} / \mathrm{O}$ (and also other mineral ratios such as $\mathrm{Si} / \mathrm{O}$, Fe/O, Helling et al. 2019a) are used as potential markers for planet formation scenarios (e.g. Helling et al. 2014; Cridland et al. 2019), the mean molecular weight is important to guide our understanding of atmospheric extensions, and the degree of ionisation shows in how far electrostatic or electromagnetic processes require attention in exoplanet atmospheres (e.g. Rodríguez-Barrera et al. 2015). The latter is an essential step towards magnetosphere studies of exoplanets (for example, Varela et al. 2018; Selhorst et al. 2020)

The paper is structured as follows. After the introduction (Sect. 1), Sect. 2 outlines our modelling approach to study the cloud properties and some key gas characteristic for the five gas planets in our sample listed in black colour in Table 1. Section 3 summarises the input. Section 4 compares the cloud properties of the different planets. Section 5 compares the planets with respect to the cloud feedback on the atmospheric gas $(\mathrm{C} / \mathrm{O})$, the mean molecular weight, and also the degree of ionisation. Section 6 looks at how the inner boundary of the General Circulation Model (GCM) affects the $\left(T_{\text {gas }}(z), p_{\text {gas }}(z)\right.$, $\left.v_{\mathrm{z}}(x, y, z)\right)$-profiles and consequently the possible cloud properties for WASP-43b. Observational implications are explored in Sect. 7, Sect. 8 presents our discussion and Sect. 9 presents our conclusions.

\section{Approach}

We adopted a two-step approach in order to examine the cloud structures of five gas planets in comparisons, similar to works on the hot Jupiters HD 189733b, HD 209458b and WASP-43b (Lee et al. 2015a; Helling et al. 2016), and the ultra-hot Jupiters WASP-18b (Helling et al. 2019a) and HAT-P-7b (Helling et al. 2019b; Molaverdikhani et al. 2020): The first modelling step produced pre-calculated 3D GCM results. These results were used as input for the second modelling step which was a kinetic cloud formation model consistently combined with equilibrium gas-chemistry calculations. We utilised $161 \mathrm{D}\left(T_{\text {gas }}(z), p_{\text {gas }}(z)\right.$, $v_{z}(x, y, z)$ )-profiles for all planets in our ensemble (Fig. 2). These profiles probe specific locations (incl. morning and evening terminators, substellar and antistellar point) on the planetary globe 

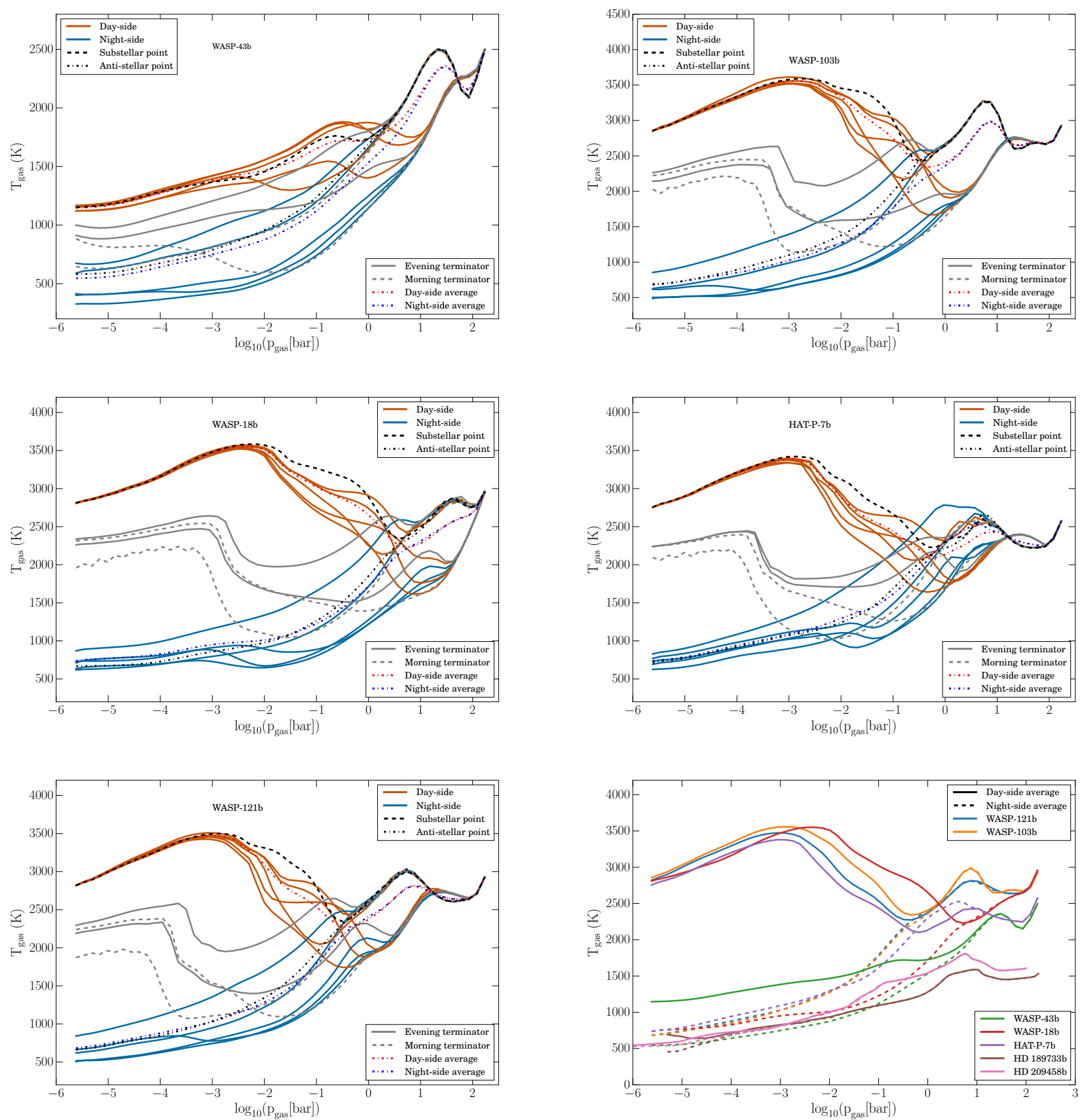

Fig. 2. Sixteen 1D $\left(T_{\text {gas }}, p_{\text {gas }}\right)$-profiles of the hot giant gas planet WASP-43b, and the ultra-hot Jupiters WASP-18b, HAT-P-7b, WASP$103 \mathrm{~b}$, and WASP-121b. The day- and nightside average profiles (lower right panel) exclude the terminators. The sampled longitudes are $\phi=0^{\circ}, 45^{\circ}, 90^{\circ}, 135^{\circ},-180^{\circ},-135^{\circ},-90^{\circ}(\phi<0$ nightside $)$, the latitudes are the equator $\theta=0^{\circ}$ and $\theta=45^{\circ}$ in the northern hemisphere. The substellar point is $(\theta, \phi)=\left(0^{\circ}, 0^{\circ}\right)$ (black dashed), the antistellar point is $(\theta, \phi)=\left(0^{\circ},-180^{\circ}\right)$ (black dash-dot), the terminators are at $\phi=90^{\circ},-90^{\circ}(\mathrm{grey}$ lines). Day/night temperature differences of $\approx 2500 \ldots 3000 \mathrm{~K}$ occur in the atmospheres of ultra-hot Jupiters.

and were distributed as depicted in Fig. 1 in Helling et al. (2019a). The same longitudes, $\phi$, and latitudes, $\theta$, are sampled for all ensemble planets studied here. The sampled longitudes are $\phi=0^{\circ}, 45^{\circ}, 90^{\circ}, 135^{\circ},-180^{\circ},-135^{\circ},-90^{\circ}$ with $\phi<0$ on the nightside. The latitudes are at the equator $\theta=0^{\circ}$ and $\theta=45^{\circ}$ in the northern hemisphere. The 3D simulations assume that the southern hemisphere is similar to the northern hemisphere. The substellar point is $(\theta, \phi)=\left(0^{\circ}, 0^{\circ}\right)$ (black dashed), the antistellar point is $(\theta, \phi)=\left(0^{\circ},-180^{\circ}\right)$ (black dash-dot), the terminators are at $\phi=90^{\circ},-90^{\circ}$ (grey lines in Fig. 2). $T_{\text {gas }}(z)$ is the local gas temperature $(\mathrm{K}), p_{\text {gas }}(z)$ is the local gas pressure (bar), and $v_{z}(x, y, z)$ is the local vertical velocity component $\left(\mathrm{cm} \mathrm{s}^{-1}\right)$. We use the solar element abundances from Asplund et al. (2009) for the undepleted element abundances.

This two-step approach has the limitation of not explicitly taking into account the potential effect of horizontal winds on cloud formation, nor the opacity of the cloud particles on the atmospheric structure. However, processes governing the formation of mineral clouds are determined by local thermodynamic properties which are the result of 3D dynamic atmosphere simulations. Cloud particle properties such as particle size or particle composition should be smeared out in longitude compared to 
the results shown here. We note that comparing Lee et al. (2015a) (without horizontal advection) and Lee et al. (2016) (including horizontal advection), the non-coupled problem is both more computationally feasible, easier to interpret and provides reasonable first order insights into the expected atmospheric cloud properties. The situation is somewhat different for photochemically triggered cloud formation. Photochemical hydrocarbon-haze production, for example, is determined by the external radiation field. We did, however, show that in the case of efficient mineral cloud formation, that hydrocarbon hazes play no role for the cloud opacity on the nightside and the terminator (Helling et al. 2020a). The dayside of all the ultra-hot Jupiters will be too hot for hydrocarbon hazes to be thermally stable.

3D GCM input. We utilised the 3D thermal structures of WASP-43b, HAT-P-7b, WASP-18b, WASP-103b, and WASP$121 \mathrm{~b}$ as described in Parmentier et al. (2018); Mansfield et al. (2018) as input for our kinetic cloud-formation simulation. These 3D GCM structures were obtained with the cloud-free SPARC/MITgcm (Showman et al. 2009) and was run for 300 Earth days, with the last 100 days used to calculate time averaged quantities. The pressure range covered is $2 \times 10^{-5}$ bar ... 100 bar. Please refer to previous paper for more details of these models.

Kinetic cloud formation. To preserve consistency for WASP-43b, HAT-P-7b, WASP-18b, WASP-103b, and WASP$121 \mathrm{~b}$, we apply the same set-up of our kinetic cloud formation model (nucleation, growth, evaporation, gravitational settling, element consumption and replenishment) and equilibrium gasphase calculations as in Sect. 2.1 in Helling et al. (2019b). Insight into numerical aspects of the solution can be found in Sect. 2.4 in Woitke \& Helling (2004). Cloud particle formation depletes the local gas phase, and gravitational settling causes these elements to be deposited, for example, in the inner (high pressure) atmosphere where the cloud particles evaporate. We use the local vertical velocity to calculate the necessary mixing timescale, $\tau_{\text {mix }} \propto v_{z}(r)^{-1}$ as outlined in Sect. 2.4 in Lee et al. (2016). Hence, $\tau_{\text {mix }} \propto K_{\mathrm{zz}}^{-1} \neq$ const. along the probed atmospheric profiles. We acknowledge that this approach may introduce limitations which, however, will affect all planets in our sample similarly. Our comparative study therefore remains valid.

Seed forming species $\left(\mathrm{TiO}_{2}, \mathrm{SiO}\right)$ also need to be considered as surface growth material, since both processes (nucleation and growth) compete for the participating elements ( $\mathrm{Ti}, \mathrm{Si}, \mathrm{O}, \mathrm{C}, \mathrm{K}$, and $\mathrm{Cl}$ in this work). We consider the formation of 16 bulk materials ([s] $=\mathrm{TiO}_{2}[\mathrm{~s}], \mathrm{Mg}_{2} \mathrm{SiO}_{4}[\mathrm{~s}], \mathrm{MgSiO}_{3}[\mathrm{~s}], \mathrm{MgO}[\mathrm{s}], \mathrm{SiO}[\mathrm{s}]$, $\mathrm{SiO}_{2}$ [s], $\mathrm{Fe}\left[\mathrm{s}\right.$ ], $\mathrm{FeO}[\mathrm{s}], \mathrm{FeS}[\mathrm{s}], \mathrm{Fe}_{2} \mathrm{O}_{3}$ [s], $\mathrm{Fe}_{2} \mathrm{SiO}_{4}$ [s], $\mathrm{Al}_{2} \mathrm{O}_{3}$ [s], $\left.\mathrm{CaTiO}_{3}[\mathrm{~s}], \mathrm{CaSiO}_{3}[\mathrm{~s}], \mathrm{C}[\mathrm{s}], \mathrm{KCl}[\mathrm{s}]\right)$ that form from 11 elements $(\mathrm{Mg}, \mathrm{Si}, \mathrm{Ti}, \mathrm{O}, \mathrm{Fe}, \mathrm{Al}, \mathrm{Ca}, \mathrm{S}, \mathrm{C}, \mathrm{K}$ and $\mathrm{Cl}$ ) by 128 surface reactions. The abundance of these 11 elements will decrease if cloud particles are forming (nucleation, growth) and increase if cloud particles evaporate. Sulphur has not been included in our present mineral cloud model. Sulphuric materials in the form of $\mathrm{S}[\mathrm{s}]$, $\mathrm{FeS}[\mathrm{s}]$, and $\mathrm{MgS}[\mathrm{s}]$ would contribute by less than $10 \%$ in a volume fraction in a solar element abundance gas (see Fig. 6 in Helling 2019).

Other kinetic cloud models emphasise the importance of the Kelvin effect on cloud formation (e.g. Powell et al. 2018; Zhang 2020). The Kelvin effect refers to the decreasing thermal stability with increasing surface curvature, hence, with decreasing particle sizes. The Fig. 3 in Goeres (1996) and in Helling \& Fomins (2013) visualise the need for a super-cooling below thermal stability as result of the decreased surface binding energy with increasing surface curvature for small particles. The effect of the resulting changing thermal stability is taken into account in our nucleation model by determining the stable molecular clusters and deriving a surface tension as outlined in Lee et al. (2015b).

\section{The different atmosphere structures}

Figure 2 summarises the 1D atmospheric $\left(T_{\text {gas }}, p_{\text {gas }}\right)$-profiles for the hot giant gas planet WASP-43b, and the four ultrahot Jupiters WASP-18b, HAT-P-7b, WASP-103b, WASP-121b. This sample of giant gas planets is homogeneous in that all $\left(T_{\text {gas }}, p_{\text {gas }}\right)$-profiles result from the same 3D GCM code. All differences that will be explored in this paper will therefore be caused by the local thermodynamic conditions, and will not be caused by differences in numerical methods or other assumptions made in different hydrodynamic simulations. Section 6 will, however, address the effect of the inner boundary for the example of WASP-43b based on results from different 3D GCMs.

Figure 2 shows that hot giant gas planets and the ultrahot Jupiters have substantially different day/night $\left(T_{\text {gas }}, p_{\text {gas }}\right)$ structures. The largest difference occurs on the dayside between these two sub-classes of gas giants. The nightsides appear more similar. All ultra-hot Jupiters sampled reach maximum gas temperatures of $\approx 3400 \mathrm{~K}$ on the dayside which suggest that no clouds will form here. This value resembles the dayside value proposed from CHEOPS observation in Lendl et al. (2020). The details of the individual temperature profiles differ, but all ultra-hot Jupiters have a comparably low nightside temperature.

Figure 2 (right lower panel) provides a comparison of the day- and the nightside averaged profiles (excluding the terminator regions). All sampled planets have a hotter dayside with a temperature inversion occurring at $p_{\text {gas }} \sim 10^{-3} \ldots 10^{-2}$ bar for the ultra-hot Jupiters. These gas temperature inversions typically display a change of $1500-2000 \mathrm{~K}$. Hot giant gas planets have a much less pronounced temperature inversion happening deeper in the atmosphere than the ultra-hot Jupiters, at about 1 bar, with a range of only less than $500 \mathrm{~K}$. In the low pressure regimes, the dayside profiles from our simulations of the ultra-hot Jupiters lie at $2700 \mathrm{~K}$, whereas the dayside profiles of the hot giant gas planets are much cooler at $1100 \mathrm{~K}$. The hot giant gas planet (WASP-43b) is the only planet in our sample that shows a net increase in temperature on the dayside with atmospheric depth. HAT-P-7b, WASP-18b, WASP-121b and WASP-103b have a roughly equivalent temperature at $10^{-6}$ and 100 bar within the GCM modelling framework utilised here. An average dayside profile of hot giant gas planets (now including WASP-43b, HD 189733b and HD 209458b for comparison) are a lot cooler than those of the ultra-hot Jupiters in our sample but exact differences vary across pressure ranges (Fig. 2, lower right panel). The average nightside temperatures of the ultra-hot Jupiters are in the temperature and pressure ranges of the average dayside profiles of the hot giant gas planets in our sample for $p_{\text {gas }}<0.1$ bar.

\section{Comparing cloud properties}

\subsection{Nucleation rate}

The nucleation rate, $J_{*}\left[\mathrm{~cm}^{-3} \mathrm{~s}^{-1}\right]$, is an essential measure for the efficiency with which cloud formation occurs, hence, with which efficiency the gas is depleted and is undergoing a phase transition leading to the formation of cloud particles. Here we 

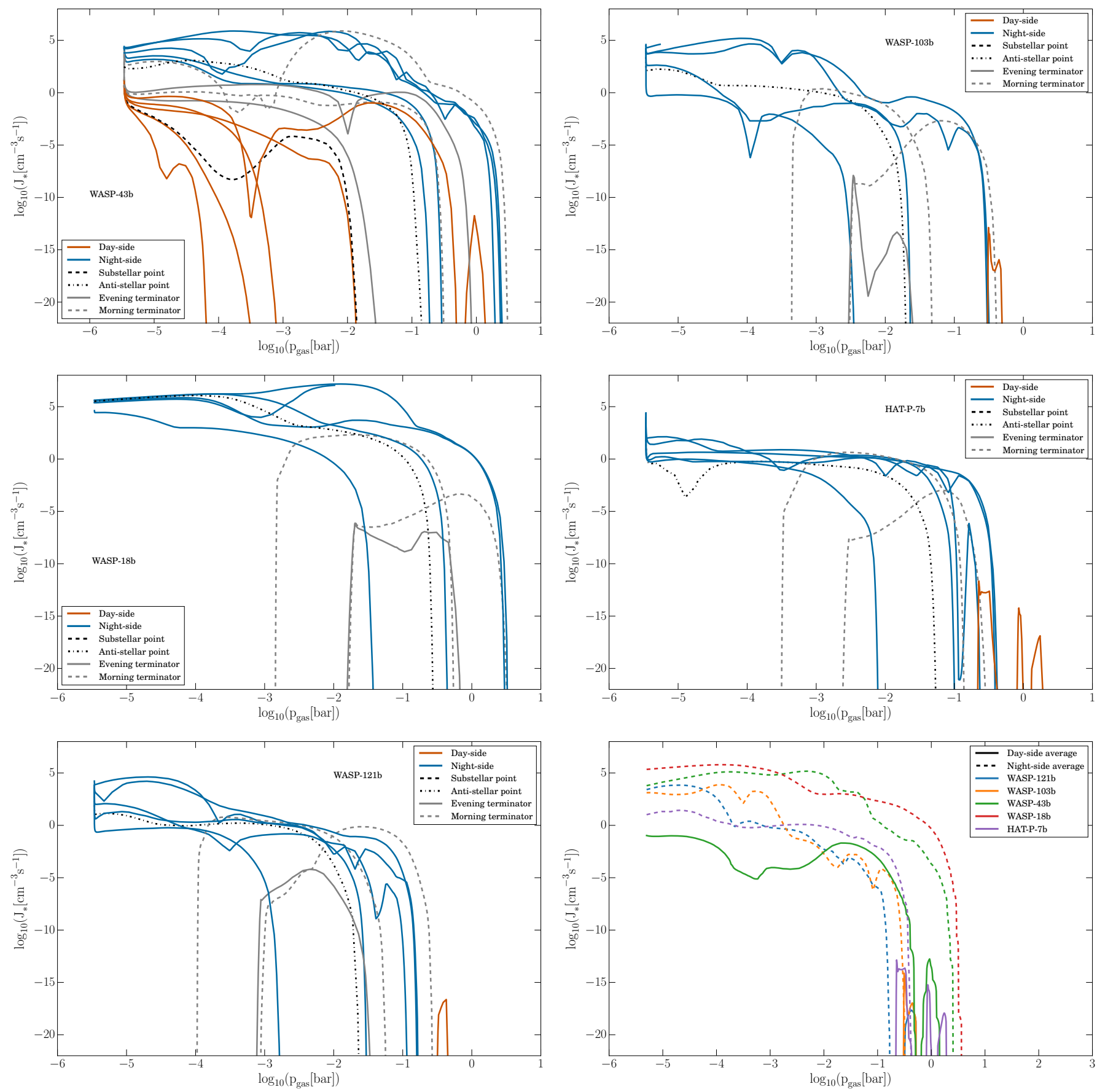

Fig. 3. Total nucleation rate $J_{*}=\sum J_{i}\left(\mathrm{~cm}^{-3} \mathrm{~s}^{-1}\right)$ for the $1 \mathrm{D}\left(T_{\text {gas }}, p_{\text {gas }}\right)$-profiles (Fig. 2) for the hot giant gas planets WASP-43b, and the ultra-hot Jupiters WASP-18b, HAT-P-7b, WASP-103b, and WASP-121bs. The colour code is similar to Fig. 2. The lower right panel shows the day (solid lines) and nightside (dashed lines) averaged seed formation rates, excluding the terminator profiles. All depicted planets show seed formation on the nightside. Only WASP-43b enables the nucleation process on the dayside efficiently.

consider the formation of mineral cloud particles which is triggered by the nucleation of $\mathrm{TiO}_{2}$ and $\mathrm{SiO}$. We analyse the cloud formation efficiency for individual profiles first (Fig. 3), before we proceed to integrated properties (Fig. 4) as the base for comparing column integrated nucleation rates for different planets according to their $T_{\text {eq }}$ and $g_{P}$ (Fig. 5).

Figure 3 demonstrates that the nightside gas temperatures are low enough that nucleation takes place for all exoplanets of our sample, hot giant gas planets and ultra-hot Jupiters. We may conclude that most if not all ultra-hot Jupiters will have clouds forming on their nightsides. Averaging over all nightside profiles (Fig. 3, lower right panel) suggests the most efficient formation of cloud condensation nuclei occurring on WASP-18b and WASP-43b with average values of $J_{*} \approx 10^{4} \ldots 10^{6} \mathrm{~cm}^{-3} \mathrm{~s}^{-1}$, and the least efficient nucleation on the nightside of WASP-43b with values of $J_{*} \approx 10^{-4} \ldots 10^{1} \mathrm{~cm}^{-3} \mathrm{~s}^{-1}$ in the upper atmosphere. The least efficient nightside nucleation in the upper atmosphere occurs in HAT-P7b. The details of the nucleation profiles depend on the local thermodynamic conditions. The nightside averaged values are higher than those retrieved with 
Ch. Helling et al.: Trending clouds in hot giant gas planets and ultra-hot Jupiters
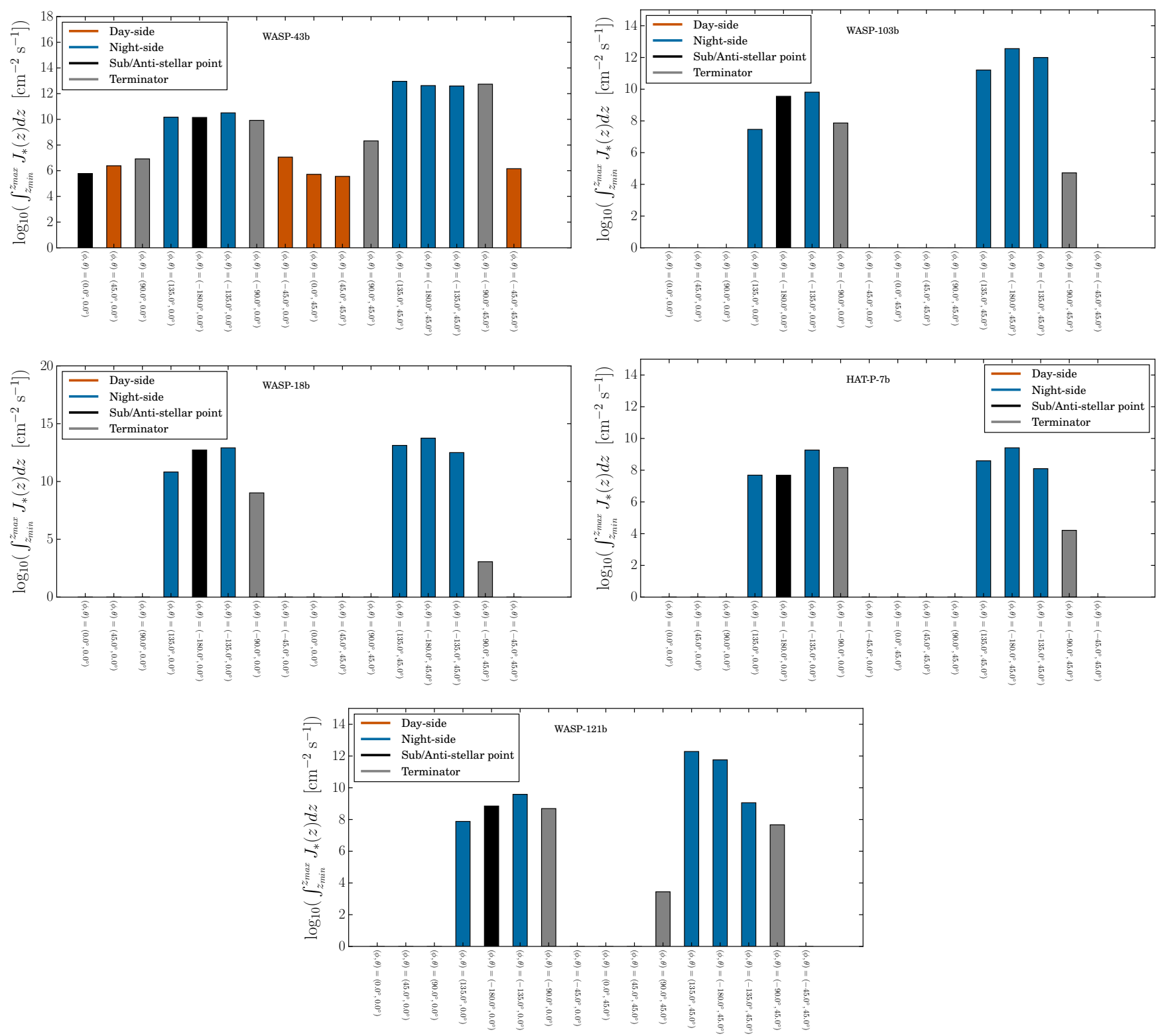

Fig. 4. Column-integrated total nucleation rates $\int_{z_{\min }}^{z_{\max }} J_{*}(z) \mathrm{d} z\left(\mathrm{~cm}^{-2} \mathrm{~s}^{-1}\right)$ for the sample planets WASP-43b, WASP-18b, HAT-P-7b, WASP-103b, and WASP-121b. WASP-103b has the highest integrated nucleation efficiency, HAT-P-7b the lowest in the nightside.
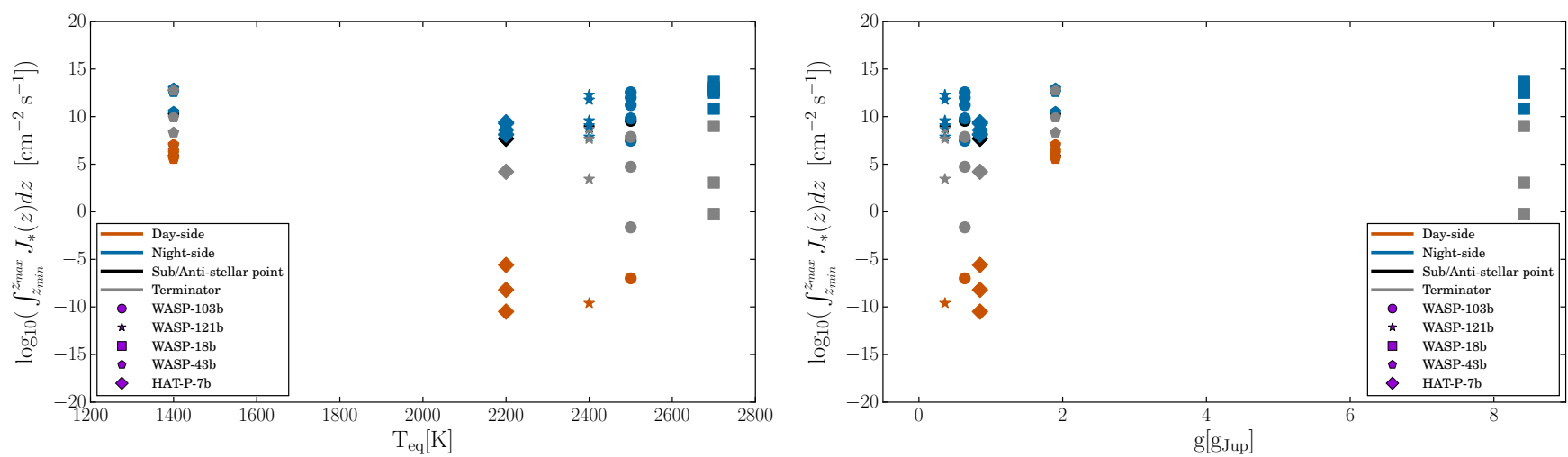

Fig. 5. Range of column-integrated nucleation rates from Fig. 4 shown for $T_{\text {eq }}(\mathrm{K})(l e f t)$ and $g\left[g_{\text {Jup }}\right]($ right $)$ for the giant gas planet WASP-43b, and the ultra-hot Jupiters HAT-P-7b, WASP-18b, and WASP-103b, WASP-121b. The WASP-18b $T_{\text {eq }}$ is offset by $+200 \mathrm{~K}$ to avoid overlap. No one value suffices to describe the rate at which cloud particles form. 
ARCiS by Min et al. (2020) for the 10 hot giant gas planets published in Sing et al. (2016). Both samples contain different planets, however.

Therefore, the only planet forming a substantial amount of cloud particles on the dayside within our sample is the hot giant gas planet WASP-43b, and a tiny nucleation peak occurs in deeper, high-pressure atmospheric regions on the dayside of WASP-121b (Fig. 3). This will have implications for the cloud particle sizes in Sect. 4.2. We have demonstrated (Lee et al. 2015a, 2016; Helling et al. 2016; Lines et al. 2018) that both the hot giant gas planets HD 189733 b and HD 209458b form clouds on the day and on the nightside. It might therefore be reasonable to conclude that hot giant gas planets can form clouds that cover the entire globe and that occupy a substantial pressure range.

To be able to compare the cloud formation efficiency in the global atmospheres of our sample planets, column integrated nucleation rates $\int_{z_{\min }}^{z_{\max }} J_{*}(z) \mathrm{d} z\left(\mathrm{~cm}^{-2} \mathrm{~s}^{-1}\right)$ are considered (Fig. 4). The left half of the plot shows the equatorial values $\left(\theta=0^{\circ}\right)$ and the right half shows northern hemisphere values $\left(\theta=45^{\circ}\right)$. The order of profiles is starting at sub-stellar point $(\phi, \theta)=\left(0^{\circ}, 0^{\circ}\right)$, move around from east to west at the equator $\theta=0^{\circ}$ (left half of the plot) and then the same for $\theta=45^{\circ}$. We note that these values average out all local information as discussed before (Fig. 3) and should be considered as guiding rather than absolute values. The colour code is the same as in Fig. 3. Nucleation is generally less efficient (on hot giant gas planets) or completely absent (ultra-hot Jupiters) on the dayside. Nucleation is more efficient in the non-equatorial hemispheres for some planets (WASP-43b, WASP-103b, WASP-121b). Nucleation is generally less efficient in the terminator regions for the ultra-hot Jupiters. The maximum nucleation efficiency is very individual for every planet. Figure 5 (left) shows the much larger spread of nucleation values for globally hotter ultra-hot Jupiters compared to the shown hot giant gas planet. This suggests that the cloud particle population will be more diverse in size on ultra-hot Jupiters than on hot giant gas planets.

\subsection{Mean particle size}

The cloud particle size, $a(\mathrm{~cm})$, is an essential value entering the opacity calculation and shows how efficient surface growth depletes the gas phase through the growth of $\mathrm{Mg} / \mathrm{Si} / \mathrm{O} / \mathrm{Fe} / \mathrm{Ti} / \mathrm{Al} /$ containing minerals. For our purpose, we show the surface averaged mean particle size, $\langle a\rangle_{\mathrm{A}}(\mathrm{cm})$, which we use for our opacity calculation in Sect. 7,

$\langle a\rangle_{\mathrm{A}}=\sqrt[3]{\frac{3}{4 \pi}} \frac{L_{3}}{L_{2}}$

with the dust moments $L_{2}$ and $L_{3}$, (Eq. (A.1) in Helling et al. 2020a). Further discussion of the different definitions of the mean particle size can be found in Appendix A of Helling et al. (2020a).

Similar to Sect. 4.1, we first present details of surface averaged mean particle size, $\langle a\rangle_{\mathrm{A}}$, in Fig. 6, before we proceed to integrated properties (Fig. 7), namely the integrated number density weighted surface averaged mean particle size,

$\left\langle\langle a\rangle_{\mathrm{A}}\right\rangle=\frac{\int_{z_{\min }}^{z_{\max }} n_{\mathrm{d}}(z)\langle a\rangle_{\mathrm{A}}(z) \mathrm{d} z}{\int_{z_{\text {min }}}^{z_{\max }} n_{\mathrm{d}}(z) \mathrm{d} z} \quad$ with $\quad n_{\mathrm{d}}(z)=\frac{\rho(z) L_{3}(z)}{4 \pi\langle a(z)\rangle_{\mathrm{A}}^{3} / 3}$

as the base for comparing column integrated mean particle sizes for different planet parameter, $T_{\mathrm{eq}}$ and $g_{\mathrm{P}}$ (Fig. 8).
Across both sides of the planets, the mean particle size, $\langle a\rangle_{\mathrm{A}}$, increases with pressure, as surface growth efficiency increases with increasing gas density (Fig. 6). Small cloud condensation seeds nucleate in the cool upper atmosphere. Due to gravitational settling these fall through the atmosphere, growing faster the deeper they fall. Both night and day show an increase in mean particle size with increasing pressure with dayside profiles always showing a larger particle radius than the nightside. Cloud particles of the size of $\langle a\rangle \approx 0.001 \mu \mathrm{m}$ reside about $2 \mathrm{~h}$ in the atmosphere where $p_{\text {gas }} \approx 10^{-5}$ bar in the terminator region of WASP-43b, but remains for $170 \mathrm{~h}$ on the nightside, which has a somewhat less extended atmosphere of higher density.

The mean particles sizes (plotted as number density weighted surface averaged, column integrated mean particle sizes, $\left\langle\langle a\rangle_{\mathrm{A}}\right\rangle$, in Fig. 7) are biggest in atmospheric regions of low nucleation efficiency. This results in a factor of 10 difference in size between day and nightside on the hot giant gas planets WASP-43b, but causes the formation of $\mathrm{cm}$-sized cloud particles at some terminator and dayside locations on the ultrahot Jupiters. Figure 8 suggests that low-mass ultra-hot Jupiters have the widest range of cloud particles sizes across their atmospheres, indicating a strong spatial in-homogeneity of these atmospheres. This includes the JWST target WASP-121b, but not the much heavier WASP-18b.

\subsection{Dust-to-gas ratio}

The dust-to-gas ratio, $\rho_{\mathrm{d}} / \rho\left(\rho_{\mathrm{d}}-\right.$ cloud particle mass density, $\rho$ - gas mass density), shows where the largest cloud particle mass is located in the atmosphere, or in broader terms, where most of the condensed mass is located. It therefore makes little sense to discuss column integrated values here. In other areas of astrophysics, $\rho_{\mathrm{d}} / \rho$, is used to measure the enrichment of gaseous environments with solid particles, such as in planet forming disks, commentary tails, the ISM, AGB star winds, super novae ejecta.

The general shape of the day- and nightside $\rho_{\mathrm{d}} / \rho$-profiles in our sample of giant exoplanets (Figs. 9 and A.1) are similar, all increasing to a maximum before falling back to zero in the inner atmosphere. The nightside of all low-mass ultra-hot Jupiters in our sample (WASP-103b, WASP-121b, HAT-P7b) have a steep increase of the atmospheric cloud particle mass load at the cloud top, reaching the maximum $\rho_{\mathrm{d}} / \rho$ within a very narrow pressure interval. Amongst the ultra-hot Jupiters, WASP-18b stands out with a shallower increase of the cloud particle mass load at the cloud top more comparable to the dayside of the gas giant WASP-43b. Figure 9 provides day- (solid line) and nightside (dashed lines) averaged $\rho_{\mathrm{d}} / \rho$ values without the terminators for the planet ensemble considered here.

For the hot giant gas planet WASP-43b in our sample, the nightside always displays a higher dust-to-gas ratio than the dayside. This is consistent with the lower nightside mean particle size. The dust-to-gas ratio sharply increases up to $4.5 \times$ $10^{-3}$ where it stays fairly level before beginning to decrease at $\sim 0.1 \ldots 1$ bar. This $\rho_{\mathrm{d}} / \rho$ values is reached if all elements such as $\mathrm{Mg} / \mathrm{Si} / \mathrm{O} / \mathrm{Fe}$ have condensed (Woitke et al. 2018), indicating that these atmospheric parts have achieved thermal equilibrium. Figure A.1 in comparison to Fig. 3 shows that, after a brief period of nucleation (which does not occur under thermal equilibrium) in the very upper atmosphere, the nightside is almost completely covered by a thick cloud at all latitude/longitude profiles, with the cloud continuing deep into the atmosphere. Most cloud particles have evaporated at 1 bar for the ultra-hot Jupiters, except for WASP-18b and the hot gas giant WASP-43b. 

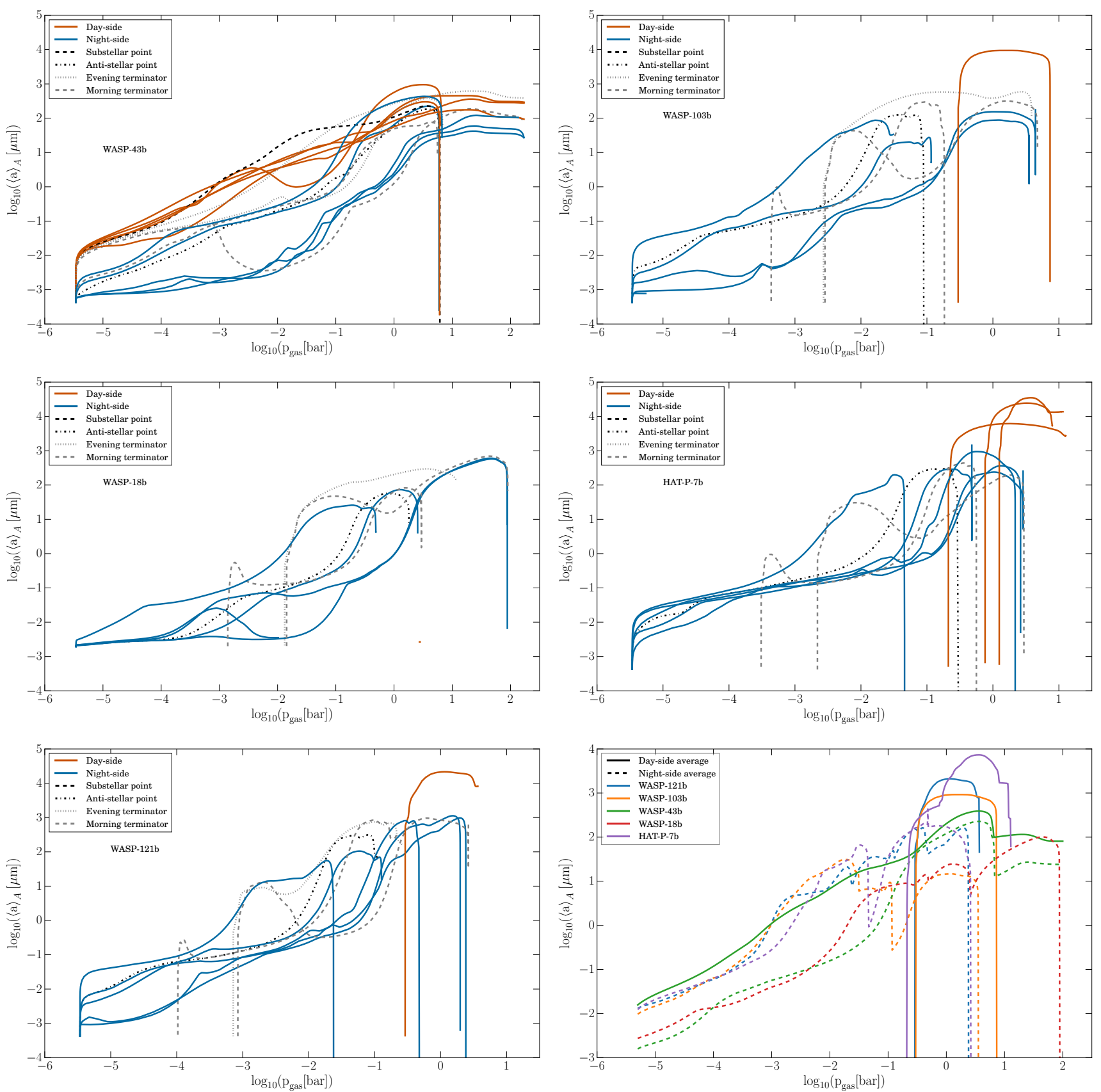

Fig. 6. Surface averaged mean particle size, $\langle a\rangle_{\mathrm{A}}[\mu \mathrm{m}]$ (Eq. (2)), for the hot giant gas planet WASP-43b, and the ultra-hot Jupiters WASP-18b, HAT-P-7b, WASP-103b, and WASP-121b. The lower right panel shows the day (solid lines) and nightside (dashed lines) averaged surface averaged mean particle size. The cloud particles sizes vary throughout the atmospheres with the largest particles occurring in the high-pressure, inner cloud layers. Cloud particles as large as $1 \mathrm{~cm}$ may occur in low numbers in the inner dayside cloud layers of ultra-hot Jupiters where nucleation is very inefficient, but present.

The northern morning terminator point, $(\phi, \theta)=\left(-90^{\circ}, 45^{\circ}\right)$, of WASP-43b (Fig. A.1) displays a short, sharp peak in dustto-gas ratio of almost $6 \times 10^{-3}$ at $10^{-3}$ bar, being considerably higher than the other profiles. This peak coincides with an influx of cold gas at the terminator, which boosts cloud particle formation. Similar peaks of often lower 'amplitude' occur for all other planets of our sample for the morning terminator. We note that substantial cloud particle mass is present at the evening terminator (grey dotted lines in the detailed plots of Fig. A.1) of some planets in our sample.

There are generally less extended (for hot giant gas planets) or no (for ultra-hot Jupiters) clouds on the dayside, shown by a lower dust-to-gas ratio, but this thinner cloud is still present across all dayside profiles and even continues deep into the atmosphere for WASP-43b. Dips in $\rho_{\mathrm{d}} / \rho$ are consistent with the dayside temperature inversions.

\subsection{Material composition of cloud particles}

The material composition of the cloud particles gives insight into the changing chemical composition of the atmosphere in which the cloud particles form, and through which they fall while they continue to grow. The 16 bulk materials considered for bulk growth are listed in Sect. 2. Here, we split these materials into 

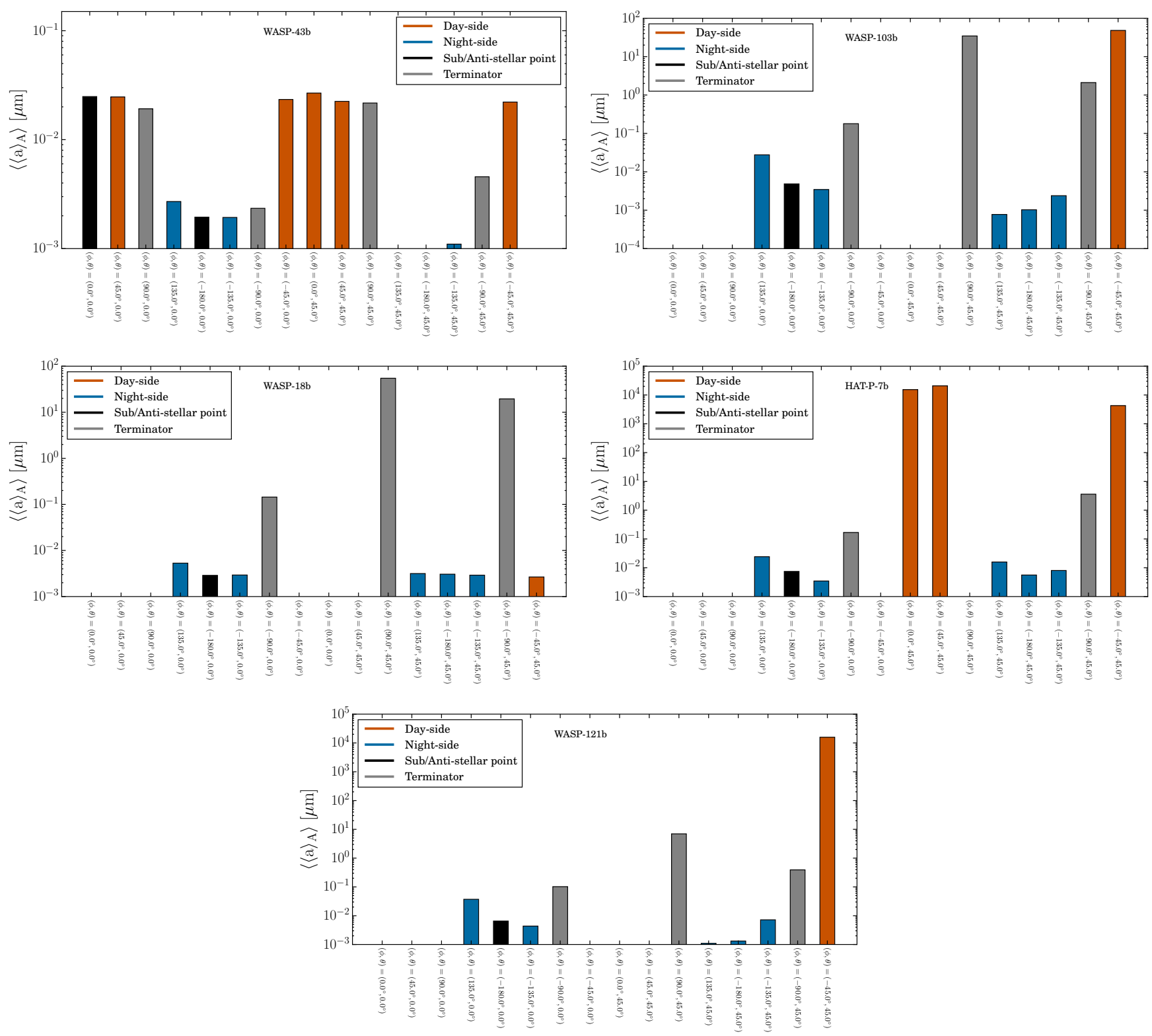

Fig. 7. Integrated, number density weighted surface averaged mean particle size, $\left\langle\langle a\rangle_{\mathrm{A}}\right\rangle=\int_{z_{\min }}^{z_{\max }} n_{\mathrm{d}}(z)\langle a\rangle_{\mathrm{A}}(z) \mathrm{d} z / \int_{z_{\min }}^{z_{\max }} n_{\mathrm{d}}(z) \mathrm{d} z$, for the giant gas planet WASP-43b and the ultra-hot Jupiters WASP-18b, HAT-P-7b, WASP-103b, and WASP-121b.
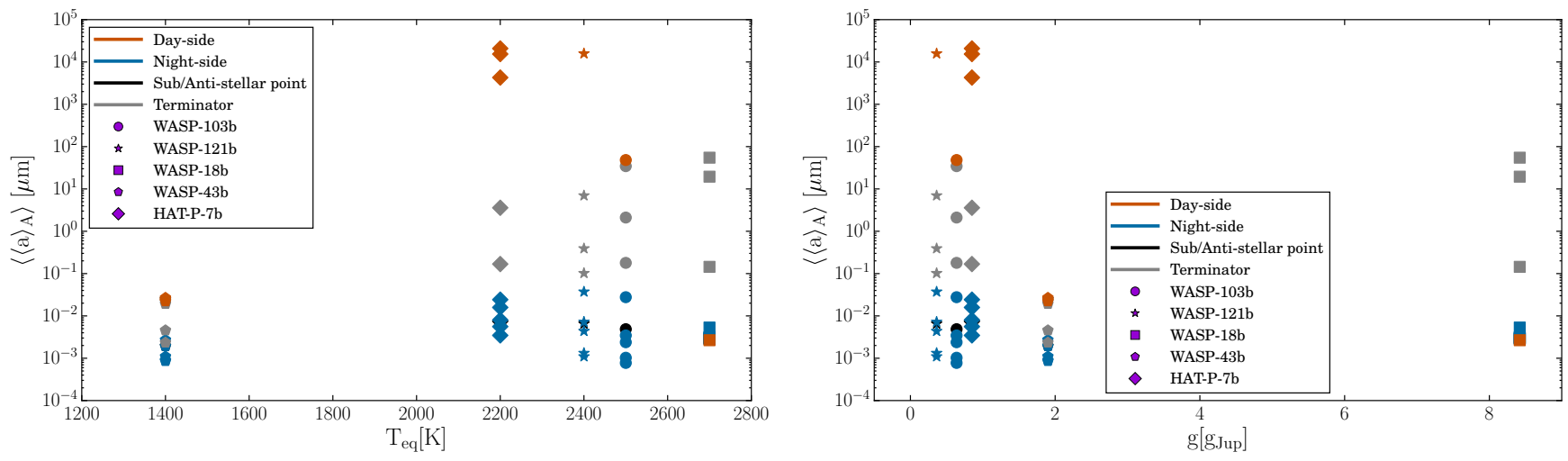

Fig. 8. Range of integrated, number density weighted surface averaged mean particle size from Fig. 7 shown for $T_{\mathrm{eq}}(\mathrm{K})(l e f t)$ and $g$ [ $\left.g_{\mathrm{Jup}}\right](r i g h t)$. The largest range of integrated cloud particles sizes occurs for ultra-hot Jupiters with small surface gravity, one being the JWST target WASP-121b. The WASP- $18 \mathrm{~b} T_{\text {eq }}$ is offest by $+200 \mathrm{~K}$ to avoid overlap. 


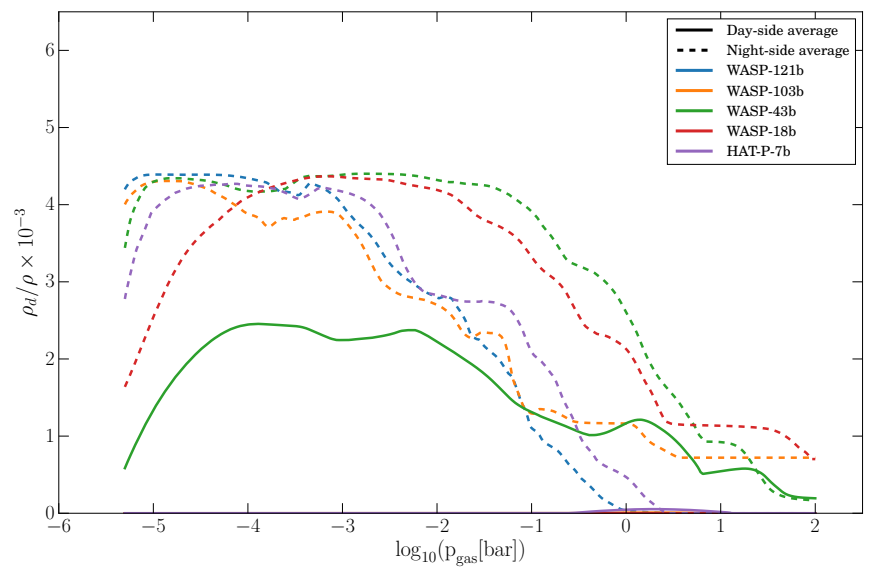

Fig. 9. Dayside (solid lines) and nightside (dashed lines) averaged dustto-gas ratios, $\rho_{\mathrm{d}} / \rho$, for the giant gas planet WASP-43b, and the ultrahot Jupiters WASP-18b, HAT-P-7b, WASP-103b, and WASP-121b. The detailed results are in Fig. A.1.

five categories of condensates: high temperature condensates, metal oxides, silicates, carbon and salts. The chemical species contained within each group are listed in Table 2. The models for WASP-18b and HAT-P-7b do not include $\mathrm{KCl}[\mathrm{s}]$. The individual material volume fractions can be found in Appendix A, the main text focuses on the material groups only.

Figure 10 shows the variation of the volume fraction for each of the material categories for each of the planets at four key points: the sub-stellar point $(\phi, \theta)=\left(0^{\circ}, 0^{\circ}\right)$, the antistellar point $(\phi, \theta)=\left(-180^{\circ}, 0^{\circ}\right)$, the equatorial morning terminator $(\phi, \theta)=\left(-90^{\circ}, 0^{\circ}\right)$, and the equatorial evening terminator $(\phi, \theta)=\left(90^{\circ}, 0^{\circ}\right)$. For the giant-gas planet WASP-43b, the upper atmosphere is dominated by silicates, making up $\sim 50 \%$ of the cloud particle volume. The next most common are metal oxides at $\sim 30 \%$, and high-temperature condensates with $\sim 20 \%$ at the hotter sub-stellar and morning terminator points. Hightemperature condensates are closer to $10 \%$ at the cooler antistellar and evening terminator points, with the extra $\sim 10 \%$ being made up by carbon materials. In the very upper atmosphere at the anti-stellar point, carbon material volume fractions are higher than high-temperature condensate volume, but decrease steadily as pressure increases from 0.01 mbar. Deeper in the atmosphere, between 10 mbar and 1 bar the fraction of metal oxides increases, and the fraction of silicates decreases as silicates evaporate. After a small increase the high temperature condensates remain constant comprising $\sim 20 \%$ of the total material volume. At 10 bar the material composition becomes dominated by high temperature condensates, with the remaining groups comprising around $5 \%$ of the composition in total.

The material volume fractions of the anti-stellar point (WASP-103b, WASP-121b and HAT-P-7b) and the equatorial evening terminator (WASP-103b, WASP-121b, HAT-P-7b and WASP-18b) follow similar variations throughout the atmosphere as seen for the same points on WASP-43b. The upper atmosphere, above 1 mbar, of the anti-stellar point of WASP-18b is dominated by metal oxides and there is a small fraction of carbon between 0.1 mbar and 1 bar. At the evening terminator there are condensates from approximately at $1 \mathrm{mbar}$ (10 mbar for WASP$18 \mathrm{~b})$ to pressures of 0.1 bar for WASP-121b and WASP-103b, 1 bar for HAT-P-7b and WASP-18b, and 10 bar for WASP-43b at which the temperature inversion occurs. Salt species are negligible for all planets in our sample. No clouds are forming at the
Table 2. The 16 bulk materials considered in our model grouped in six categories.

\begin{tabular}{ll}
\hline \hline Condensate group & Materials included \\
\hline Metal oxides & $\mathrm{SiO}[\mathrm{s}], \mathrm{SiO}_{2}[\mathrm{~s}], \mathrm{MgO}[\mathrm{s}]$, \\
Silicates & $\mathrm{FeO}[\mathrm{s}], \mathrm{Fe}_{2} \mathrm{O}_{3}[\mathrm{~s}]$ \\
& $\mathrm{MgSiO}_{3}[\mathrm{~s}], \quad \mathrm{Mg}_{2} \mathrm{SiO}_{4}[\mathrm{~s}]$, \\
Carbon & $\mathrm{CaSiO}_{3}[\mathrm{~s}], \mathrm{Fe}_{2} \mathrm{SiO}_{4}[\mathrm{~s}]$ \\
High temperature & $\mathrm{C}[\mathrm{s}]$ \\
Condensates & $\mathrm{TiO}_{2}[\mathrm{~s}], \quad \mathrm{Fe}[\mathrm{s}], \quad \mathrm{Al}_{2} \mathrm{O}_{3}[\mathrm{~s}]$, \\
Salts & $\mathrm{CaTi}_{3}[\mathrm{~s}], \mathrm{FeS}[\mathrm{s}]$ \\
\hline
\end{tabular}

Notes. [s] indicates condensate materials.

sub-stellar point and at the equatorial morning terminator for all the ultra-hot Jupiters (hence, the respective panels are empty).

Figure 10 also allows to see the pressure range, and thus how much of the atmosphere, over which cloud condensates are forming in the frame of our model. There are cloud particles forming throughout most of the atmosphere of WASP-43b, ranging from $p \approx 0.001-0.01$ mbar . . 10 bar, for all four atmosphere profiles. For the ultra-hot Jupiters WASP-121b, WASP-103b and HAT-P-7b the anti-stellar point shows clouds forming between $p \approx 0.001-0.01$ mbar ... 0.1 bar, however the morning terminator shows clouds forming in a narrower region of the atmosphere between $p \approx 0.1-1$ mbar...0.1 bar. WASP-18b shows a similar pattern to the other ultra-hot Jupiters with the exception of cloud particles at the morning terminator forming deeper in the atmosphere between $p \approx 1-10$ bar.

The material volume fractions for all planets 'oscillate' between groups as pressure is increased. This is caused by a 'switching' back and forth associated with the evaporation of individual species, which frees up elements that are then consumed by thermally stable species in other condensate groups: The transition between $\mathrm{Fe}_{2} \mathrm{SiO}_{4}[\mathrm{~s}]$ and $\mathrm{Fe}[\mathrm{s}]$ as can be seen more clearly in Figs. A.2-A.5. As a general rule, the volume fractions of silicates and carbon decrease whereas volume fractions of metal oxides and high temperature condensates increase as pressure increases as result of their thermal stability. In all profiles, metal oxides become more common than silicates at higher pressures.

Figure 11 shows the normalised column integrated volume fractions,

$\langle V\rangle_{\text {norm }}=\frac{\int_{z_{\min }}^{z_{\max }} \frac{V_{s}(z)}{V_{\text {tot }}(z)} \mathrm{d} z}{\sum_{i} \int_{z_{\min }}^{z_{\max }} \frac{V_{i}(z)}{V_{\text {tot }}(z)} \mathrm{d} z}$,

where $i$ runs through each of the condensate groups listed in Table 2, for the same four points as shown in Fig. 10. These values provide an average cloud composition at this particular point, however it does not contain the details on the local pressure and material composition variation and so should be used only as a guiding value. Both the anti-stellar point and the morning terminator of all planets show that metal oxides and silicates together dominate the cloud composition making up between $\sim 60-70 \%$ of the total volume of the cloud particles. The remaining volume is comprised predominantly of high temperature condensates and small fractions of carbon. The sub-stellar point of WASP-43b shows metal oxides and silicates comprise $~ 30 \%$ of the material composition each, with the remaining $\sim 40 \%$ 

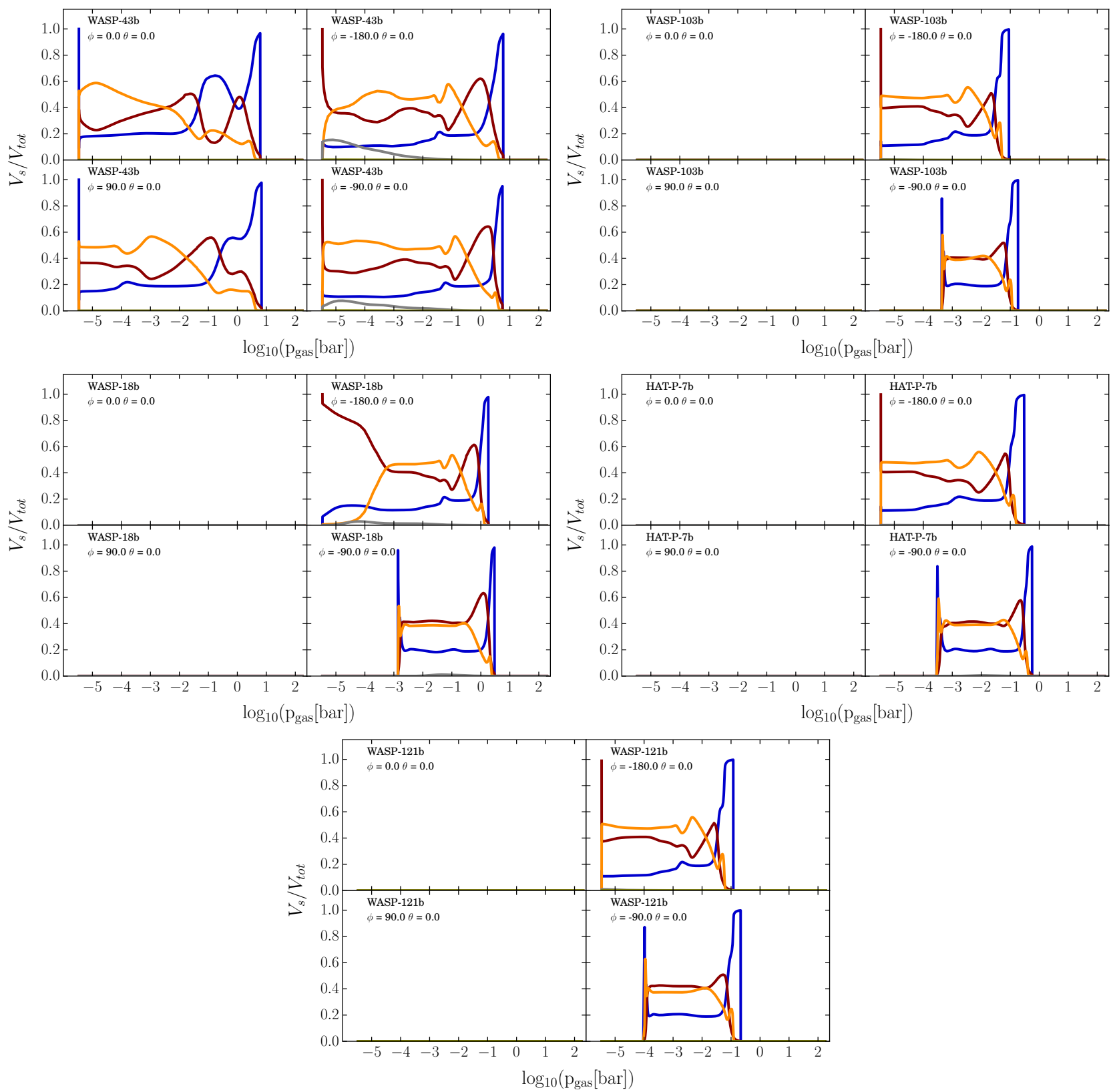

Fig. 10. Volume fractions $V_{\mathrm{s}} / V_{\text {tot }}$ of the material groups as defined in Table 2 (blue: high temperature condensates, red: metal oxides, orange: silicates, grey: carbon, olive: salts). Sub-stellar point: $(\phi, \theta)=\left(0.0^{\circ}, 0.0^{\circ}\right)$, anti-stellar point: $(\phi, \theta)=\left(-180.0^{\circ}, 0.0^{\circ}\right)$, equatorial morning terminator: $(\phi, \theta)=\left(-90.0^{\circ}, 0.0^{\circ}\right)$, equatorial evening terminator: $(\phi, \theta)=\left(90.0^{\circ}, 0.0^{\circ}\right)$. There are no salt condensate species included for WASP-18b and HAT$\mathrm{P}-7 \mathrm{~b}$ due to their very low abundance. Empty panels represent profiles without cloud formation.

being high temperature condensates. The high temperature condensates, metal oxides and silicates are almost equal in their contribution to the volume of WASP-43b's evening terminator clouds.

\section{The comparison of characteristic global gas phase properties}

\subsection{The carbon-to-oxygen ratio}

The carbon-to-oxygen ratio is often used in astrophysics to decide if an object is carbon rich, that is has more carbon than oxygen, or oxygen rich. Most of the exoplanet host stars will be oxygen-rich as the majority of stars in the Universe are main sequence stars today. Once low-mass stars develop into AGB stars, the star will become carbon-rich. The measurement of the stellar carbon and the oxygen abundance relies on highresolution spectra, a technique which has only recently begun to be available in exoplanet research through instruments such as CARMENES, PEPSI, and CRIRES+. So far, however, only the mere presence of atoms has been shown (e.g. Casasayas-Barris et al. 2019; Yan et al. 2020) and detailed abundance measurements are compromised by atmospheric clouds (e.g. Nikolov et al. 2018; Carter et al. 2020). Here, we focus on the local C/O which is determined by how much oxygen is locked in cloud particle materials such as $\mathrm{MgSiO}_{3}[\mathrm{~s}], \mathrm{MgO}[\mathrm{s}], \mathrm{Al}_{2} \mathrm{O}_{3}$ [s] etc. $\mathrm{A}$ similar exercise can be conducted for other element (or mineral) ratios as for example shown in Helling et al. (2019a) (their Figs. A.3. ff.). 

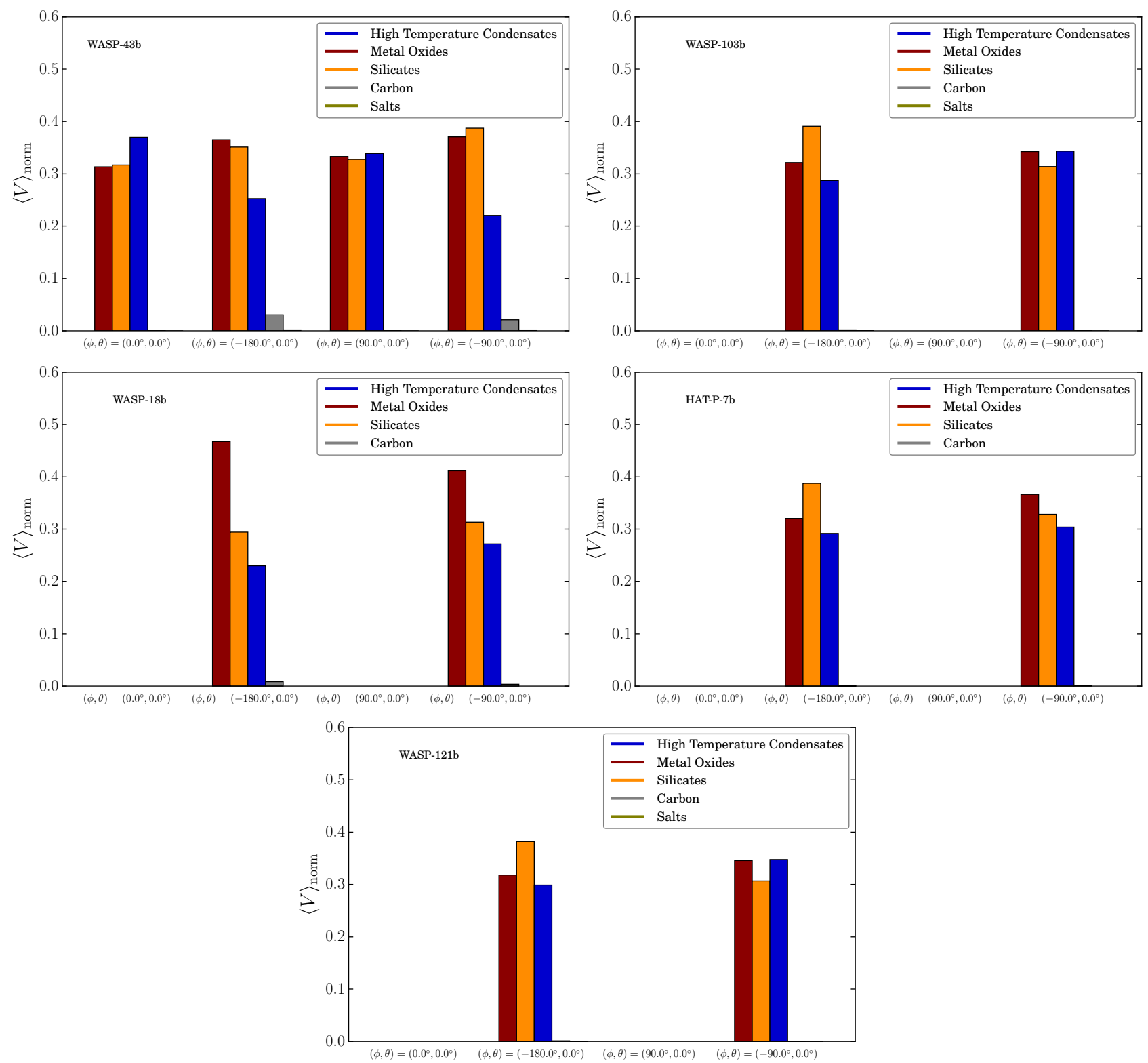

Fig. 11. Normalised column integrated volume fractions $\langle V\rangle_{\text {norm }}=\int_{z_{\min }}^{z_{\max }} \frac{V_{s}(z)}{V_{\text {tot }}(z)} \mathrm{d} z / \sum_{i} \int_{z_{\min }}^{z_{\max }} \frac{V_{i}(z)}{V_{\text {tot }}(z)} \mathrm{d} z$, where $s$ is the given material group and $i$ runs over all the material groups as defined in Table 2 (blue: high temperature condensates, red: metal oxides, orange: silicates, grey: carbon, olive: salts). Sub-stellar point: $(\phi, \theta)=\left(0.0^{\circ}, 0.0^{\circ}\right)$, anti-stellar point: $(\phi, \theta)=\left(-180.0^{\circ}, 0.0^{\circ}\right)$, equatorial morning terminator: $(\phi, \theta)=\left(-90.0^{\circ}, 0.0^{\circ}\right)$, equatorial evening terminator: $(\phi, \theta)=\left(90.0^{\circ}, 0.0^{\circ}\right)$. The relative abundance of the metal oxides, silicates and high temperature condensates is comparable for the substellar point of the ultra-hot Jupiters WASP-103b, HAT-P-7b and WASP-121b.

As demonstrated in Fig. 12 (top) all cloud-forming profiles have on average a $\mathrm{C} / \mathrm{O}$ ratio larger than the undepleted, solar value of 0.55 in the upper atmosphere indicating oxygen depletion by cloud particle condensation. This result holds also for the individual 1D profiles of the 3D atmospheres (Fig. A.6). The atmosphere turns more oxygen-rich at $\sim 1$ bar ( 0.1 bar for WASP121b) in atmospheric regions where cloud particles efficiently evaporate and, hence, enrich the local gas phase with all elements previously locked within the cloud materials, including oxygen. Hence, cloud particle transport elements into the deeper atmosphere. The strongest enrichment occurs for WASP-43b, WASP-103b and HAT-P-7b for the dayside profiles with a small number of big cloud particles evaporating.

All planets in our sample can be expected to have a large range of $\mathrm{C} / \mathrm{O}$ values from 0.54 to 0.75 on the nightside (blue lines in Fig. A.6). The average nightside $\mathrm{C} / \mathrm{O}$ values (Fig. 12, top) are similar among the ultra-hot Jupiters, except for WASP$18 \mathrm{~b}$ which is the most massive planet with a larger bulk density than any of the other gas planets in our sample.

Transmission spectra probe the terminator regions of the atmosphere which, according to our study, are very likely to differ in the $\mathrm{C} / \mathrm{O}$ (grey dashed and grey dotted lines in Fig. A.6). Especially when considering the asymmetry in clouds, thus comparing the $\mathrm{C} / \mathrm{O}$ for just above the opaque cloud level shows that $1 \mathrm{D}$ retrievals will fail to capture the global $\mathrm{C} / \mathrm{O}$ ratio.

Our computations confirm the conclusion by Baxter et al. (2020) that both, hot and ultra-hot Jupiters are likely to have $\mathrm{C} / \mathrm{O}<0.8$, but we cannot confirm that these planets have a global solar or near solar $\mathrm{C} / \mathrm{O}$. The solar (or original) $\mathrm{C} / \mathrm{O}$ can only be expected for the cloud free parts of the dayside. Lower than solar 

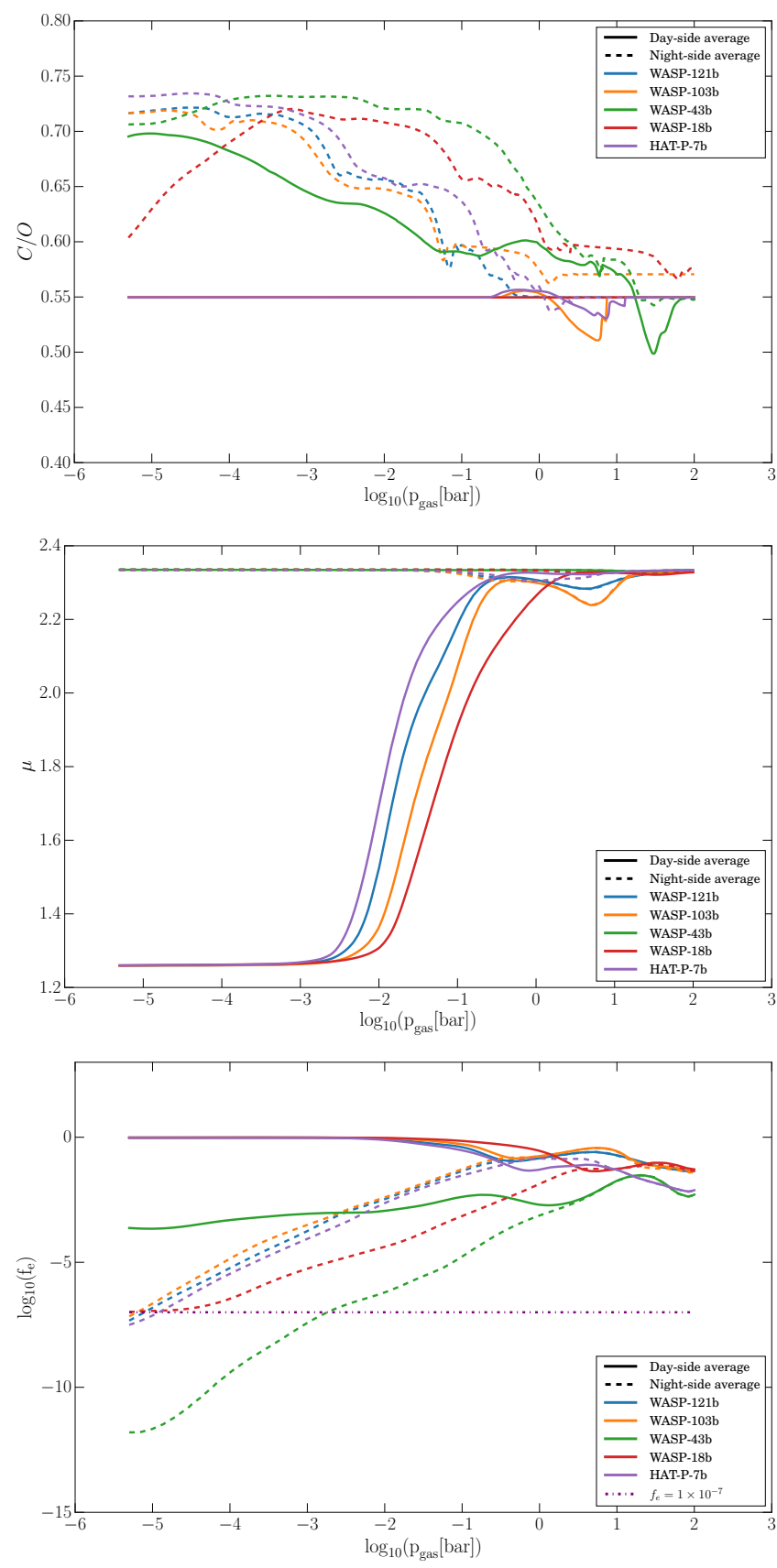

Fig. 12. Carbon-to-oxygen ratio (C/O, top), atmospheric mean molecular weight, $\mu$ (middle), the degree of thermal ionisation, $f_{\mathrm{e}}=p_{\mathrm{e}} /\left(p_{\text {gas }}+p_{\mathrm{e}}\right)($ bottom $)$ for the hot giant gas planet WASP-43b, and the ultra-hot Jupiters WASP-18b, HAT-P-7b, WASP-103b, and WASP121b. The solar value $\mathrm{C} / \mathrm{O}=0.54$ is plotted in dashed purple (top). The dash-dot purple line shows $f_{\mathrm{e}}=1 \times 10^{-7}$ as a threshold for plasma behaviour (bottom). All ultra-hot Jupiters have dayside thermal ionisation $f_{\mathrm{e}}>10^{-4}$ suggesting an extended dayside ionosphere. The detailed results for the individual planets are provided in Figs. A.1-A.8.

$\mathrm{C} / \mathrm{O}$ would either point to an effective mass transport through the atmosphere (see $\mathrm{C} / \mathrm{O}$ spikes $<0.55$ on Fig. A.6) or to an originally higher oxygen or lower carbon content of the atmospheric gas. Cridland et al. (2020) suggest that young giant gas planets should be expected to be more oxygen rich than older planets that have migrated through the disk already. This would be caused by accretion of more icy material from high above the mid-plan of the planet-forming disk than originally assumed.

\subsection{Mean molecular weight}

The mean molecular weight, $\mu$, defined as the mean mass of a particle in a gas, is an important quantity to know as it can be used to transform the local gas pressure into the local gas density via the ideal gas law. It, hence, enters calculations of transmission depth being expressed in terms of pressure scale heights $\left(H=k_{\mathrm{B}} T_{\mathrm{eq}} /\left(\mu g_{\mathrm{P}}\right)\right.$; e.g. Alexoudi et al. 2020) or for deriving a cloud top pressure for defining an isothermal transit radius (Heng 2019). A constant value of $\mu$ is often assumed when running a 3D GCM (see introduction in Drummond et al. 2018) as it is beneficial computationally. Zhang \& Showman (2017) show that a changing bulk composition of the atmosphere (hence, a changing mean molecular weight) leads to a decreasing zonal wind velocity with increasing $\mu$, causing the planetary atmosphere to develop a more banded structure and a larger day/night temperature contrast. We demonstrate here that assuming a constant atmospheric bulk composition $(\mu=$ const.) globally may not be valid in all cases. Figure 12 (middle) summarises the results in terms of dayside and nightside averaged mean molecular weights (terminators excluded) for the hot Jupiter WASP-43b, and for the ultra-hot Jupiters WASP-103b, WASP-121b, HAT-P-7b, and WASP-18b. Figure A.7 provides the detailed results for each planet individually.

For WASP-43b, the hot giant gas planet in our sample, the mean molecular weight remains approximately constant $(\mu=2.328 \ldots 2.337)$ throughout the entire atmosphere on both the day and night side of the planet, consistent with a molecular hydrogen dominated atmosphere. The ultra-hot Jupiters present a very different story with the day- and nightside value of $\mu$ varying substantially across the global atmosphere. In the upper atmosphere, above $1 \mathrm{mbar}$, of the ultra-hot Jupiters the nightside value is $\mu \approx 2.3$, whereas the dayside has a value of $\mu \approx 1.3$. This difference is caused by the large temperature difference between the day and night sides of the planet seen in Fig. 2 which leads the dayside being highly ionised, in addition to molecular hydrogen being unable to form.

The changing mean molecular weight which results from the local temperature effect on the gas-phase composition, results in substantial changes in the geometrical extension of the atmosphere around the globe. The details are summarised in Appendix B where the substantial effect of the changing mean molecular weight on the hydrostatic pressure scale height is shown.

\subsection{Degree of ionisation}

The degree of ionisation (Fig. 12 (bottom) and Fig. A.8), $f_{\mathrm{e}}=n_{\mathrm{e}} / n_{\text {tot }}\left(n_{\text {tot }}-\right.$ total gas number density, $n_{\mathrm{e}}-$ electron number density), provides a first insight into potential plasma behaviour within the atmospheres of exoplanets, including the possibility of forming an ionosphere and a magnetosphere in the presence of a potential magnetic field. A value of $f_{\mathrm{e}}>10^{-7}$ is postulated as a threshold for transitioning from gas to plasma behaviour (Rodríguez-Barrera et al. 2015). Here, we consider thermal ionisation for the calculation of $f_{\mathrm{e}}$ only.

The high gas temperatures on the dayside of the ultra-hot Jupiters results in a highly ionised upper atmosphere with $f_{\mathrm{e}}$ approaching almost 1 , and a less ionised lower atmosphere with $f_{\mathrm{e}}=10^{-2} \ldots 10^{-1}$. The nightside gas temperatures of the ultra-hot Jupiters are sufficient for a partially ionised atmosphere where $f_{\mathrm{e}} \approx 10^{-6} \ldots 10^{-2}$, where the increasing thermal ionisation is in line with the increased gas temperature 
deeper in the atmosphere. The giant gas planet WASP-43b has dayside and nightside gas temperatures $\sim 1000-1500 \mathrm{~K}$ and $\sim 500 \mathrm{~K}$ less than at the same points of the ultra-hot Jupiters (Fig. 2) and thus the atmosphere is much less ionised. The dayside has $f_{\mathrm{e}} \approx 10^{-4}$ throughout the entire atmosphere and the nightside has $f_{\mathrm{e}}=10^{-13}-10^{-3}$ increasing with atmospheric depth.

The daysides of all the ultra-hot Jupiters (WASP-103b, WASP-121b, HAT-P-7b, WASP-18b) and the giant gas planet WASP-43b are sufficiently ionised by thermal processes such that an extended ionosphere is present. Such an ionosphere is geometrically more extended for ultra-hot Jupiters compared to hot giant gas planets according to the geometrical extension of the atmosphere (Appendix B). The degree of ionisation will further be enhanced on the dayside by the XUV radiation and stellar energetic particles of the host stars which will affect the outermost layers of the atmosphere. The nightside will not be affected by the stellar XUV and SEPs, but by the galactic cosmic rays. CRs have little effect in the atmospheric ionisation (Rimmer \& Helling 2013), but can open kinetic pathways to form complex hydrocarbon molecules (Rimmer et al. 2014; Barth et al. 2020). Koskinen et al. (2014) demonstrate that the outer atmosphere of the hot gas giant HD 209458b is magnetically coupled to a global magnetic field that may be present. The magnetic coupling amplifies with height in HD 209458b due to the increasing effect of photoionisation. Barth et al. (2020) show that photochemistry amplifies the day/night asymmetry due to the high host star's radiation flux (XUV, FUV, SEPs) for tidally locked, close-in planets. Therefore, in the presence of a magnetic field (Zaghoo \& Collins 2018; Cauley et al. 2019), a magnetosphere may affect the atmosphere of hot giant gas planets globally (as on WASP-43b and HD 209733b), but it will have a strongly asymmetric effect on the atmosphere of ultra-hot Jupiters due to their strongly asymmetric ionosphere (as on WASP-103b, WASP121b, HAT-P-7b, WASP-18b). The asymmetry of an extended magnetosphere may be detectable as a bow shock as result of the interaction with the host-star wind (Lai et al. 2010; Vidotto et al. 2010,2011 ) or through radio transit observation (Selhorst et al. 2020) in the future.

The best candidates for detecting a magnetosphere could be the ultra-hot Jupiters WASP-103b, WASP-121b and HAT-P-7b if using the hydrostatic scale height as a first guiding estimate (Fig. B.1). Taking into account the interaction with the stellar wind, Vidotto et al. (2011) proposes WASP-18b (amongst others) as target for detecting a bow-shock. The coupling of the ionised part of a globally circulating atmosphere with a potentially existing exoplanet magnetic field may cause a current system to emerge that reduces the angular velocity at high latitudes and generate an auroral emission comparable to what has been suggested for brown dwarfs (Nichols et al. 2012). Rogers (2017) present MHD simulations for a giant gas planet with a day/night temperature difference of $\Delta T=1000 \mathrm{~K}$, a prescribed temperature profile in order to solve the Saha equation, and the planetary parameters of HAT-P7b. The arising Lorentz forces disrupt strong eastward atmospheric winds on such a dayside causing an oscillating pattern with a characteristics time scale (11.5 days for the chosen set up in Rogers 2017).

\section{The effect of the inner boundary on GCM results for the example of WASP-43b}

Simulations using GCMs requires extensive computational resources, in particular if the radiation hydrodynamics is solved consistently with the gas chemistry and actual cloud formation modelling. Therefore, it is unsurprising that all exoplanet models apply a cloud parameterisation of some sort (Dobbs-Dixon \& Agol 2013; Charnay et al. 2018; Mendonça et al. 2018; Lines et al. 2019; Roman et al. 2021; Parmentier et al. 2021). Grids of GCM simulations are often run as completely cloud free or as opacity species only with cloud properties derived in postprocessing (e.g. Kataria et al. 2015; Parmentier et al. 2018). Consequently, little time has been afforded to run extensive test on other assumptions, for example, the inner boundary. Recently, Carone et al. (2020) proposed that very deep layers (down to 700 bar) need to be considered to fully capture the emergence of waves that sculpt the observable climate in hot Jupiters that rotate faster than 1.5 days. This situation may be the case for WASP-43b but also generally for ultra-hot Jupiters (see Table 1, most tidally locked ultra-hot Jupiter are expected to have rotation periods faster than 1.5 days). The possible importance to resolve deeper layers and a sufficiently long simulation times has been further confirmed by Wang \& Wordsworth (2020); Showman et al. (2020). Here, we take the opportunity to offer a first discussion on what effect the choice of the inner boundary may have on the cloud coverage of the hot giant gas planets, WASP-43b.

\subsection{Approach}

In what follows, we compare the WASP-43b results from two 3D GCM simulations: Parmentier's version of SPARC/MITgcm (see Sect. 2) and Carone 3D GCM. We follow the same approach as outlines in Sect. 2, but additionally utilised the input data from the Carone 3D GCM simulations (Carone et al. 2020) for WASP-43b. We point out differences between the two 3D MITgcm versions below. However, an understanding of why the models differ in detail will require a more extensive comparison study which is outwith the scope of this paper. We demonstrate that shifting the inner boundary does extend the cloud layer into deeper, high pressure regimes. This raises the question about additional heating by backwarming from the cloud later which will, in turn, affect the thermal ionisation of the gas.

Also the Carone 3D GCM simulation for WASP-43b is cloud-free. It uses simplified radiative transfer via Newtonian cooling compared to a non-grey binned radiative transfer in Parmentier et al. (2018). Thus, the temperature in the Carone model is less well constrained with a temperature uncertainty of up to $100 \mathrm{~K}$. Carone et al. (2020) has a dynamically active atmosphere that extends deeper downwards to pressures of 700 bar, allowing for the formation of deep wind jets. Carone et al. (2020) uses several additional measures to stabilise the lower boundary at $p>10$ bar. These are: temperature convergence to the interior adiabat, a shorter convergence time scale $\tau_{\text {conv }}=10^{6} \mathrm{~s}$ and drag between 550 and 700 bar (Carone et al. 2020, Sect. 2.3). These measures are chosen ensure that the atmosphere is in a dynamical steady state from the top to the bottom.

The SPARC/MITgcm runs of WASP-43b used here use a free slip, impermeable boundary condition situated at 200 bars and no drag other than numerical dissipation is added (see Showman et al. 2009, for the detailed setup used). Because of the computational cost of running non-grey radiative transfer compared to the much faster Newtonian cooling, the models are integrated for 300 days, which is shorter than the 2000 days of Carone et al. (2020). With such a short integration timescale, the deep layers of the model are not yet equilibrated. The statistical steady-state reached at the photosphere is therefore dependent on the assumption that whatever circulation would develop in the deep layers 
of the planet is not strongly affecting the photospheric flow, an assumption recently challenged by Carone et al. (2020).

Different conditions in the deep atmosphere are proposed to induce a different climate regime compared to the thermal photosphere. Waves can travel upwards from the optically thick to the optically thin atmosphere regime. The different climate regime naturally leads to a larger day-to-night side temperature contrast and much colder (cloud free) night side temperatures compared to other GCMs. Thus, despite the different level of approximations chosen in the Paramentier SPARC/MITgcm and in the Carone GCM, we investigate if the proposed wave connection between the atmosphere at greater depth (>100 bar) affect cloud formation and chemistry higher up in the atmosphere where these effects maybe observable.

\subsection{The effect of the GCMs inner boundary on cloud formation in atmospheres of giant gas planets}

We explore this question by comparing our cloud formation results for the Paramentier SPARC/MITgcm and the Carone GCM. We also explore the effect on $\mathrm{C} / \mathrm{O}$ and on the local thermal ionisation as direct effects of cloud formation and thermodynamics. Figure 13 (top) shows the two sets of ( $\left.T_{\text {gas }}, p_{\text {gas }}\right)$-profiles, both showing temperature inversions at $p_{\text {gas }} \sim 0.1$ bar. Generally, the Carone models demonstrate that the cloud formation will extend deeper into the atmosphere due to the increasing thermal stability with increasing pressure for increasing temperature as the result of moving the inner boundary to higher pressures. The nucleation rate night profiles (Fig. 13, second row) have similar shape and values for both models, with the Carone model enabling seed formation deeper into the lower atmosphere. Consequently, the grain size night profiles (Fig. 13, 3rd row) have similar shapes and values for both models, and so does the cloud particle mass load in term of the dust-to-gas, $\rho_{\mathrm{d}} / \rho$ (Fig. 13, 4th row).

The cloud formation on the dayside differs more between the two models than on the night side, reflecting the dayside differences in the $\left(T_{\text {gas }}, p_{\text {gas }}\right)$ profiles. The inner dayside cloud ( $\mathrm{p}_{\mathrm{gas}}>0.1$ bar) forms more efficiently in the Carone model, hence, more cloud particles form such that they remain smaller up to $p_{\text {gas }} \approx 1$ bar compared to the Parmentier model. At higher pressure, the surface growth of the gravitational settling cloud particles becomes more efficient in the Carone model (Fig. 13, right column) than in the Parmentier models (Fig. 13, left column) as the local densities are simply higher, hence the inner cloud has more and bigger cloud particles in the Carone model on the dayside and on the nightside.

Figure 14 (top) shows that the thermodynamics of the atmosphere affects also the material compositions of the cloud particles (for more details see Appendix C), suggesting that it is important to extend the 3D GCM models not only into lower atmospheric pressure regions where the stellar irradiation will affect the gas phase photo-chemically, but also towards higher pressures at the inner boundary. The cloud particle material composition will affect the element depletion of the gas phase locally, which we represent here in terms of the carbon-to-oxygen ratio (Fig. 14, 2nd row). Overall, the $\mathrm{C} / \mathrm{O}$ values are comparable or even similar, but can differ in detail. For example is $\mathrm{C} / \mathrm{O}<0.6$ at $p_{\text {gas }} \approx 10^{-2}$ bar at the substellar point in the Carone model but $\mathrm{C} / \mathrm{O} \approx 0.68$ in the Parmentier model at the same pressure. The reason is that the element depletion is affected by the dynamics of the cloud particle formation which is determined by the cloud particle history in that a smaller particle will fall less fast into an atmosphere than a bigger particle.

Both models predict a partially ionised dayside through thermal ionisation (Fig. 14, 3rd row), but little ionisation on the nightside, hence, a magnetosphere should only be expected to form on the dayside from both models. We conclude this comparison by noting that the geometrical extension and hence the mean molecular weight are comparable in both models for a given pressure level (Fig. B.5).

In this section, we studied how the treatment of the inner boundary and that of the inner atmosphere will affect the cloud properties, the $\mathrm{C} / \mathrm{O}$ and the thermal degree of ionisation. We conclude that the qualitative findings such as the presence of clouds, average $\mathrm{C} / \mathrm{O}$ or degree of ionisation are in reasonable agreement between the two 3D atmosphere simulations.

While the results discussed above remain qualitatively the same, the details of the material composition of the clouds (see top row of Fig. B.5) appears significantly differently for both the anti-stellar and sub-stellar point. Here, the temperature and density differences are the largest between the Parmentier and Carone model (Fig. 14 top row). Thus, the night side clouds are composed of metal oxides and are geometrically more extended in the Carone model, whereas in the Parmentier model the clouds are composed of silicates and are thinner.

There also appears to be a difference for the substellar points of the two models. For pressures greater than $\sim 10^{-1}$ bar this difference is easily explained by the differences between the thermal profiles of the models, with the Parmentier model being warmer in this region. However, for pressures between $10^{-4}$ $10^{-1}$ bar the two models have temperatures within $100 \mathrm{~K}$ of one another for the same pressure. Thus, it is surprising that in this region the Carone model produces clouds dominated by hightemperature condensates, whereas the clouds in the Parmentier model are mostly made of silicates and metal oxides. To explore this Fig. 15 shows the $S=1$ curves for all cloud condensates in our model with the substellar profiles for the two models (Carone in blue and Pamrmentier in red) over-plotted. This clearly shows that the slightly higher temperature of Carone models in this region puts it above the thermal stability curve for the magnesium bearing species $\mathrm{MgO}[\mathrm{s}], \mathrm{MgSiO}_{3}[\mathrm{~s}], \mathrm{Mg}_{2} \mathrm{SiO}_{4}[\mathrm{~s}]$ as well as that of $\mathrm{Fe}$ and $\mathrm{SiO}[\mathrm{s}]$, which make up the majority of the cloud material in the Parmentier models (Fig. B.3). This leaves only the high-temperature condensates of $\mathrm{TiO}_{2}[\mathrm{~s}], \mathrm{CaSiO}_{3}[\mathrm{~s}], \mathrm{CaTiO}_{3}[\mathrm{~s}], \mathrm{Al}_{2} \mathrm{O}_{3}[\mathrm{~s}]$ to be thermally stable. This explains the high-temperature condensate peak before the drop in temperature at 0.1 bar in the Carone model, as here only $\mathrm{CaTiO}_{3}$ [s] and $\mathrm{Al}_{2} \mathrm{O}_{3}$ [s]. Although we stress here the results shown in Fig. 15 are for solar abundances at all temperatures and pressures, and do not reflect our full kinetic model, where cloud formation depletes the gas phase of certain elements and hence changes the supersaturation ratios of condensate species bearing these elements.

As a summary, we note that generally the night sides of tidally locked exoplanets are the most susceptible to effects of the inner boundary. The nightside temperatures are set by the interior temperature and horizontal heat transport originating from the irradiated day side (Thorngren et al. 2019). Since horizontal heat transport is less efficient for ultra-hot Jupiters (Komacek \& Showman 2016; Komacek et al. 2017), assumptions about the interior temperature and thus the lower boundary will become important for these planets. Recently, the temperature of the deep interior (at 200 bar) was invoked to explain observations of Fe and $\mathrm{Mg}$ in the ultra-hot WASP-121b, which led to constraints of the interior temperature $T_{\text {int }}=500 \mathrm{~K}$ (Sing et al. 2019, Fig. 13). 
Ch. Helling et al.: Trending clouds in hot giant gas planets and ultra-hot Jupiters
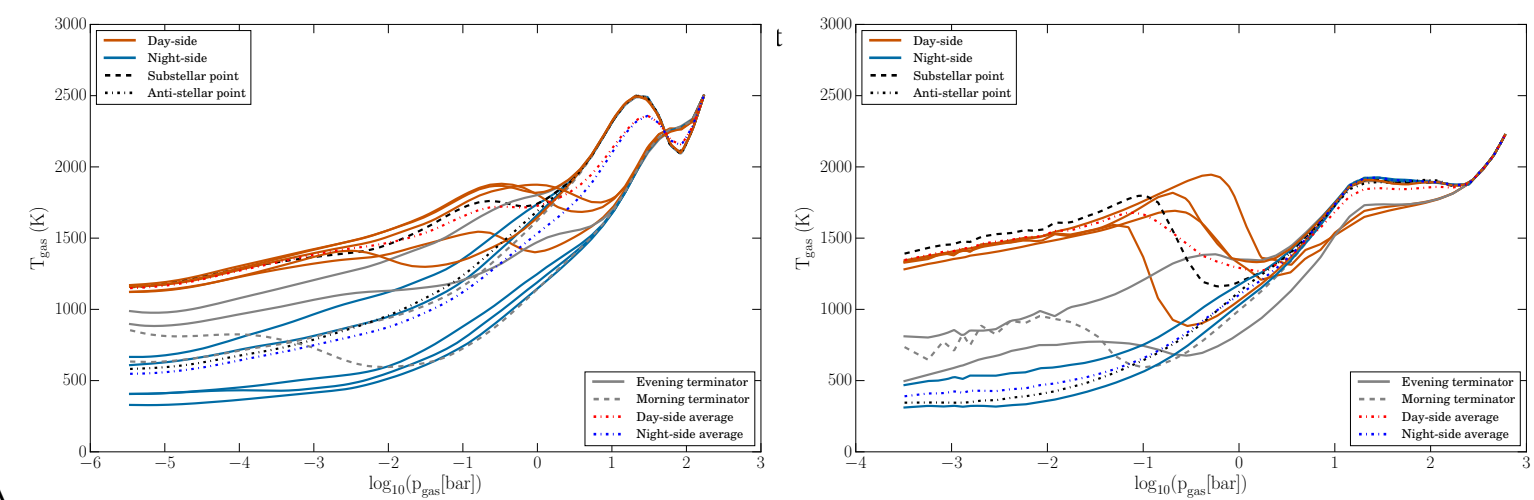

A
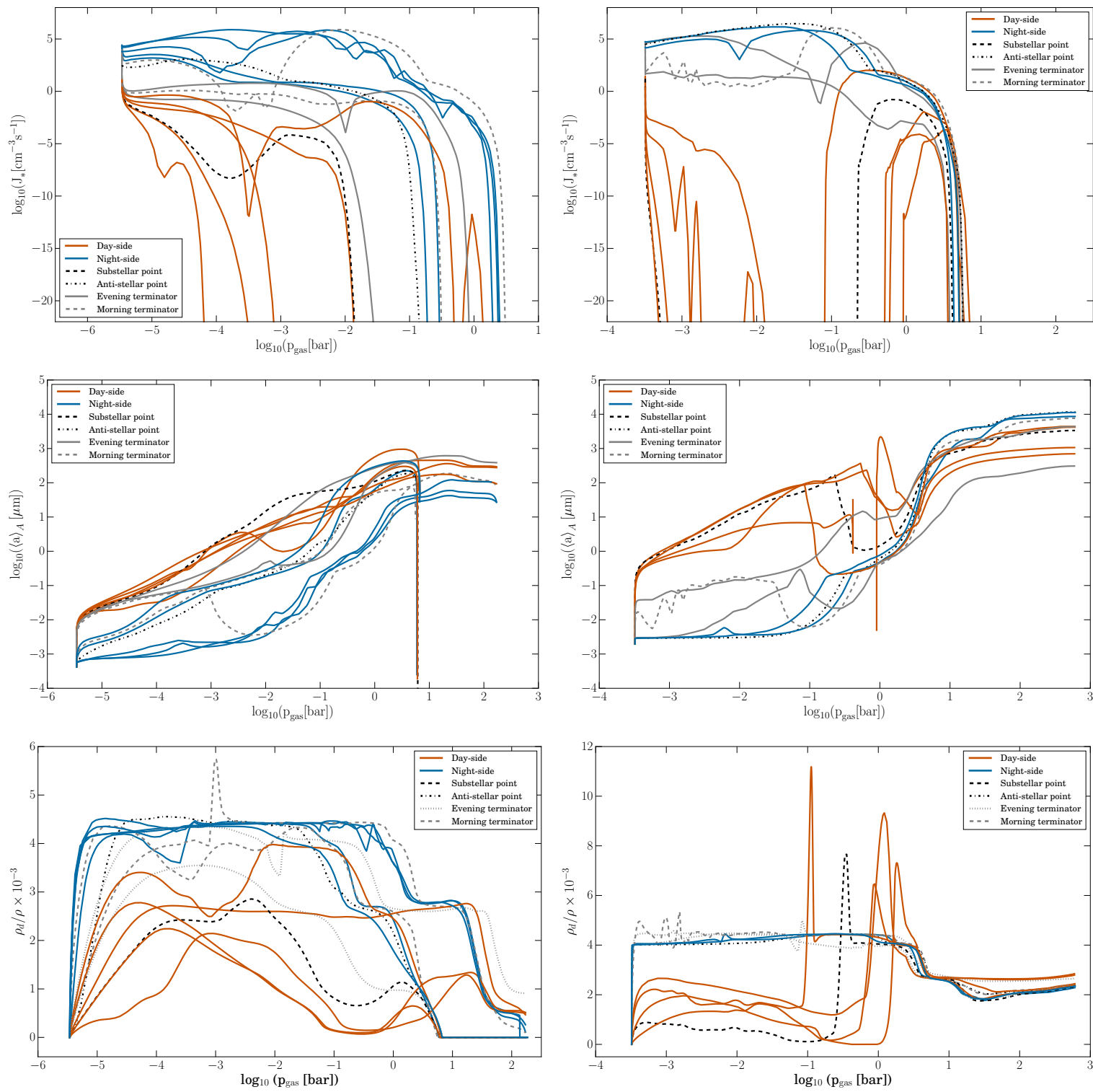

Fig. 13. Effect of the inner boundary of the $3 \mathrm{D}$ GCM models on the $\left(T_{\text {gas }}, p_{\text {gas }}\right)$-profiles and the local cloud properties $J_{*},\langle a\rangle_{A}$, and $\rho_{\mathrm{d}} / \rho$ for the giant gas planet example WASP-43b. Left: based on the 1D thermodynamic profiles from Parmentier et al. Right: based on the 1D thermodynamic profiles from Carone et al.

An important next step to shed further light on model differences would be to set up both 3D GCMs with the very same numerical parameters for the inner boundary and the radiative transfer treatment of the atmosphere. This is, however, outwith the scope of this paper.

\section{Observational implications}

We now take a look at the spectroscopic properties of the clouds for each of four ultra-hot Jupiters (WASP-18b, WASP103b, WASP-121b, and HAT-P-7b) and one gas giant planet 

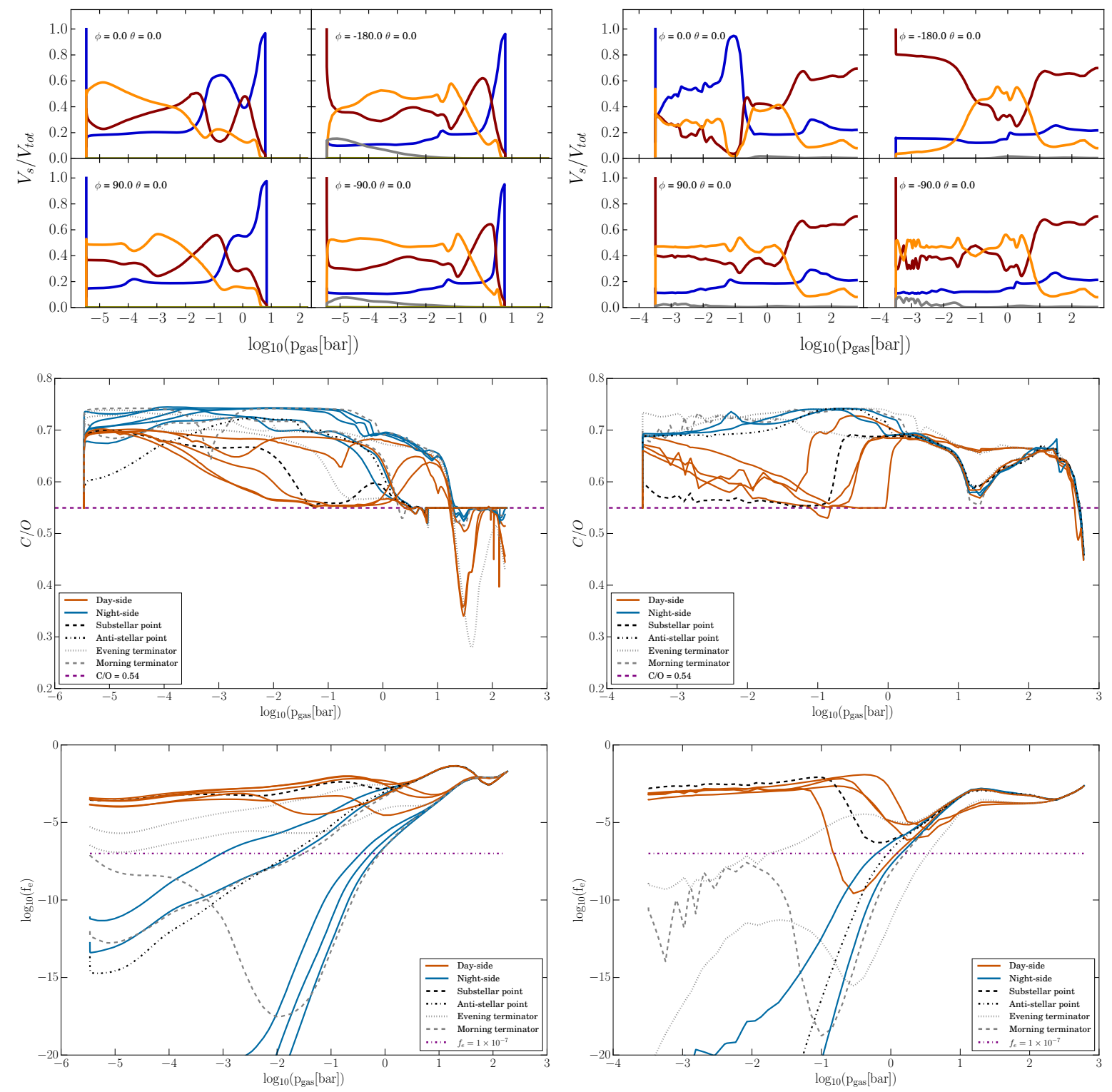

Fig. 14. Effect of the inner boundary of the 3D GCM models on the grouped $V_{\mathrm{s}} / V_{\mathrm{tot}}$, the local $\mathrm{C} / \mathrm{O}$, and the degree of ionisation for the giant gas planet example WASP-43b. Left: Parmentier et al. Right: Carone et al. The differences in the cloud particles material fractions result from the different local temperatures of the two GCM models, with the Carone model nightside being cooler than the Parmentier model nightside.

(WASP-43b), at four points around the equator $\left(\theta=0.0^{\circ}\right)$, the sub-stellar and anti-stellar points $\left(\phi=0.0^{\circ}, 180.0^{\circ}\right)$, and the morning and evening terminators $\left(\phi=90^{\circ},-90^{\circ}\right)$. The terminator profiles are indicative of what could be seen in emission for secondary eclipse, and in transmission spectroscopy. The anti-stellar point is difficult to observe, but is representative of nightside conditions, which is where cloud formation is very efficient and the most similarity in cloud structure between the planets occur. To investigate the atmosphere observable for both of these techniques (transmission and emission) we must know what pressure levels are optically thin, that is $\tau<1$; for example in transmission, the atmosphere deeper than this level is not visible to observers.

The optical depth along some path from $z_{0}$ to $z$ for a given wavelength is defined as

$\tau\left(\lambda, z-z_{0}\right)=\int_{z_{0}}^{z} \kappa\left(\lambda, z^{\prime}\right) \rho\left(z^{\prime}\right) \mathrm{d} z^{\prime}$ where $\kappa$ is the extinction coefficient per unit atmospheric mass. For our atmospheres we use cloud spectral properties along vertical profiles, hence $z_{0}=0$ is the top of the atmosphere and $z$ is the depth into the atmosphere. We then interpolate the pressure at the depth where $\tau=1$ to get the pressure at which the clouds ceases to be optically thin. Extinction coefficients for the cloud particles are calculated using Mie theory (Mie 1908; Bohren \& Huffman 1983) using the surface average particle radius from the moments as defined in Eq. (1) with corresponding number density as discussed in Helling et al. (2020a):

$n_{\mathrm{d}, \mathrm{A}}=\frac{\rho L_{2}^{3}}{L_{3}^{2}}$.

Mixed material refractive indices are determined using effective medium theory with the Bruggeman mixing rule (Bruggeman 1935). Individual cloud species refractive indices are the same as in Helling et al. (2019b), with the addition 


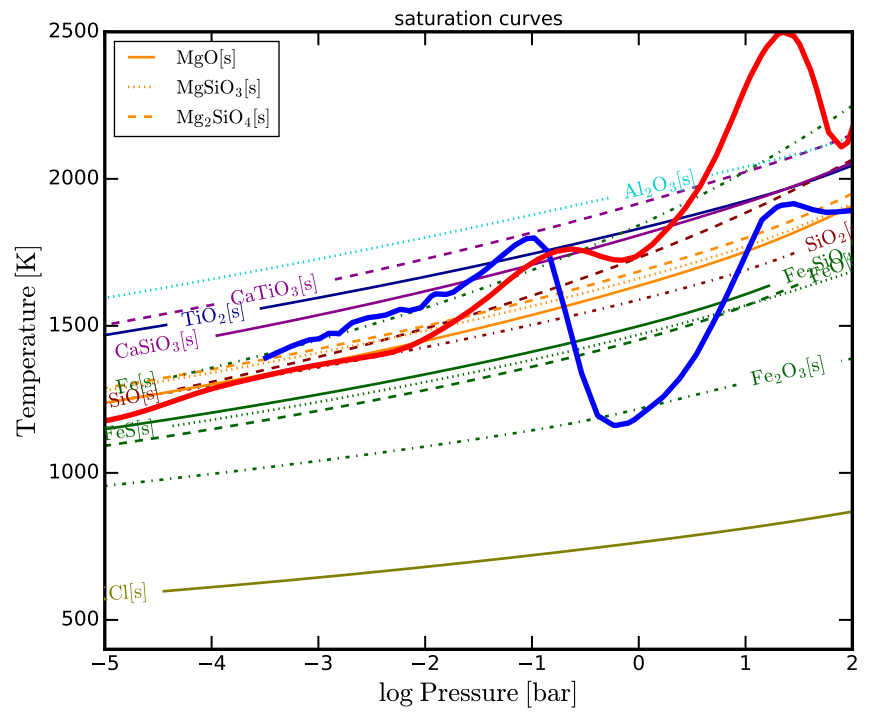

Fig. 15. Sub-stellar ( $T_{\text {gas }}, p_{\text {gas }}$ )-profiles from the Carone (blue, extended inner boundary) and the Parmentier (red, standard inner boundary) GCM runs for WASP-43b. The comparison to the thermal stability curves (supersaturation ratio $S=1$ for solar element abundances) of selected solid materials shows that the local temperature differences at $p_{\text {gas }} \approx 1$ bar support our finding for the cloud particle material composition differences between the two models (Fig. 14). We note that the $S=1$ curves do not represent our full kinetic model approach and are provided here for the purpose of visualisation.

of $\mathrm{KCl}$ from (Palik 1985) for all planets except WASP18$b$ and HATP-7b. To account for the effects of non-spherical cloud particles we include a Distribution of Hollow Spheres (DHS) (Min et al. 2005; Samra et al. 2020). Hollow spheres are defined by a structure of a vacuous core and a mantle containing the material volume of a compact sphere of radius $\langle a\rangle_{\mathrm{A}}$, with volume fractions of materials as appropriate for that atmospheric layer. A distribution of these particles with different fractions of volume being the vacuum core are then averaged over. This represents well the distributions of irregularly shaped particles for protoplanetary disks, both in the Rayleigh regime and for larger particles (Min et al. 2003, 2008; Min 2015), and has now also been implemented in atmospheric models ATRES (Stolker et al. 2017), PetitCODE (Mollière et al. 2015, 2017), ARCiS (Ormel \& Min 2019; Chubb et al. 2020) and also in retrievals PetitRADTRANS (Mollière et al. 2019).

For ultra-hot Jupiter exoplanets there is significant difference in extension between the day and the nightside of the planet (see Appendix B), furthermore as the stellar light passes through the atmosphere at a slant geometry (Fortney 2005), there is a nonzero width of atmosphere probed around the terminator. The angle (in longitude) to which transit spectra are sensitive has been determined in recent works using both a parameterised estimation (Caldas et al. 2019), and by examining the impact of a full radiative transfer model (Lacy \& Burrows 2020; Pluriel et al. 2020), both found the angle for these planets to vary between $10^{\circ}-40^{\circ}$, that is between $\pm 5^{\circ}-20^{\circ}$ around the terminator. From our studied sample, WASP-18b representing the lower end of this range and HAT-P-7b and WASP-103b representing the upper end (see Lacy \& Burrows 2020, their Fig. 3 top left). As our approach produces 1D cloud profiles of selected longitude-latitude points, with a spacing in longitude of $45^{\circ}$ our grid spacing is too wide to meaningfully integrate along line-of-sight trajectories through the atmosphere. We therefore chose to use vertically integrated optical depth of the clouds and apply a correction for the effect of slant geometries. In order to take into account the effect of slant geometry we use an correction factor calculated for a hydrostatic atmosphere, using the work of Fortney (2005), the slant geometry method of determining the optical depth adjusts the vertically integrated $\tau$ to

$\tau_{\mathrm{s}}=\tau \sqrt{\frac{2 \pi R_{\mathrm{p}}}{H_{\mathrm{p}}}}$,

where $R_{\mathrm{p}}$ is the radius of the planet, and $H_{\mathrm{p}}$ is the hydrostatic pressure scale height, $H_{\mathrm{p}}=(k T) /\left(\mu \mathrm{m}_{\mathrm{H}} g\right)$, with $k$ the Boltzmann constant, $m_{\mathrm{H}}$ the atomic mass unit, $g$ the gravitational acceleration of the planet, and $\mu$ the mean molecular weight, for which we use $\mu=2.3$ for all substellar points. For the anti-stellar points and terminators we choose $\mu=1.3$ (with the exception of WASP-43b which continues to use the sub-stellar value) as suggested by our results in Fig. 12 (middle panel). This correction assumes that cloud properties along the vertical 1D profile are not substantially different from points probed in the optically thin atmosphere along the line-of-sight. This has been previously been investigated for more dense grids of longitude and latitudes (for example for HAT-P-7b in Helling et al. 2019b and for WASP43b in Helling et al. 2020a), with especially rapid change in cloud properties around the terminators of ultra-hot Jupiters. Particularly at the morning terminator the pressure at which clouds form is a function of angle from the morning terminator, with the further day-ward points having clouds only at deeper levels and higher pressures, thus the optical depths of these profiles are likely affected by our assumption here. For calculations of transmission spectra it is clear that a fully three dimensional calculation is necessary as shown in Pluriel et al. (2020) and Lacy \& Burrows (2020), we leave such a full analysis to future works as previously noted, because of our wide grid spacing.

\subsection{The $p\left(\tau_{s}(\lambda)=1\right)$-levels for a hot gas giants and four ultra-hot Jupiters}

Figure 16 shows the results of optical thick pressure levels including the effect of slant geometry for compact (solid) and non-spherical (dashed) cloud particles. Full plots for vertically integrated optical depth alongside the slant values for each planet are found in Fig. A.9. Only WASP-43b is sufficiently cool on the dayside to have clouds present at the sub-stellar point, and as it is substantially different from all of the other planets, it will be discussed separately in Sect. 7.2. For the ultra-hot Jupiters, the inefficient heat re-distribution from the dayside to the nightside along with global circulation initiated by planetary rotation causes the evening terminators to be too hot for cloud formation, in addition to the nightside. Evidence for the effects of partial cloud coverage around the terminator has been found in retrievals (Line \& Parmentier 2016; Lacy \& Burrows 2020). Clouds are present at the morning terminator $\left((\phi, \theta)=\left(-90^{\circ}, 0^{\circ}\right)\right)$ in all ultra-hot Jupiters (Fig. 16, top left panels), but remain confined by the temperature inversion to deeper atmospheric levels of $p>10^{-3}$ bar such that the atmosphere above would not be affected by cloud opacity. This results in cloud particles sizes of $10 \ldots 100 \mathrm{~m}$ at $\tau_{\mathrm{s}}=1$ for $\lambda<10 \mathrm{~m}$ in the ultra-hot Jupiters in comparison to $0.01 \ldots 0.1 \mu \mathrm{m}$ at $\tau_{\mathrm{s}}=1$ for $\lambda<10 \mathrm{~m}$ for the hot giant WASP-43b according to our present models. The material properties vary in accordance to the local temperatures. For WASP-103b, HAT-P-7b, WASP-18b 


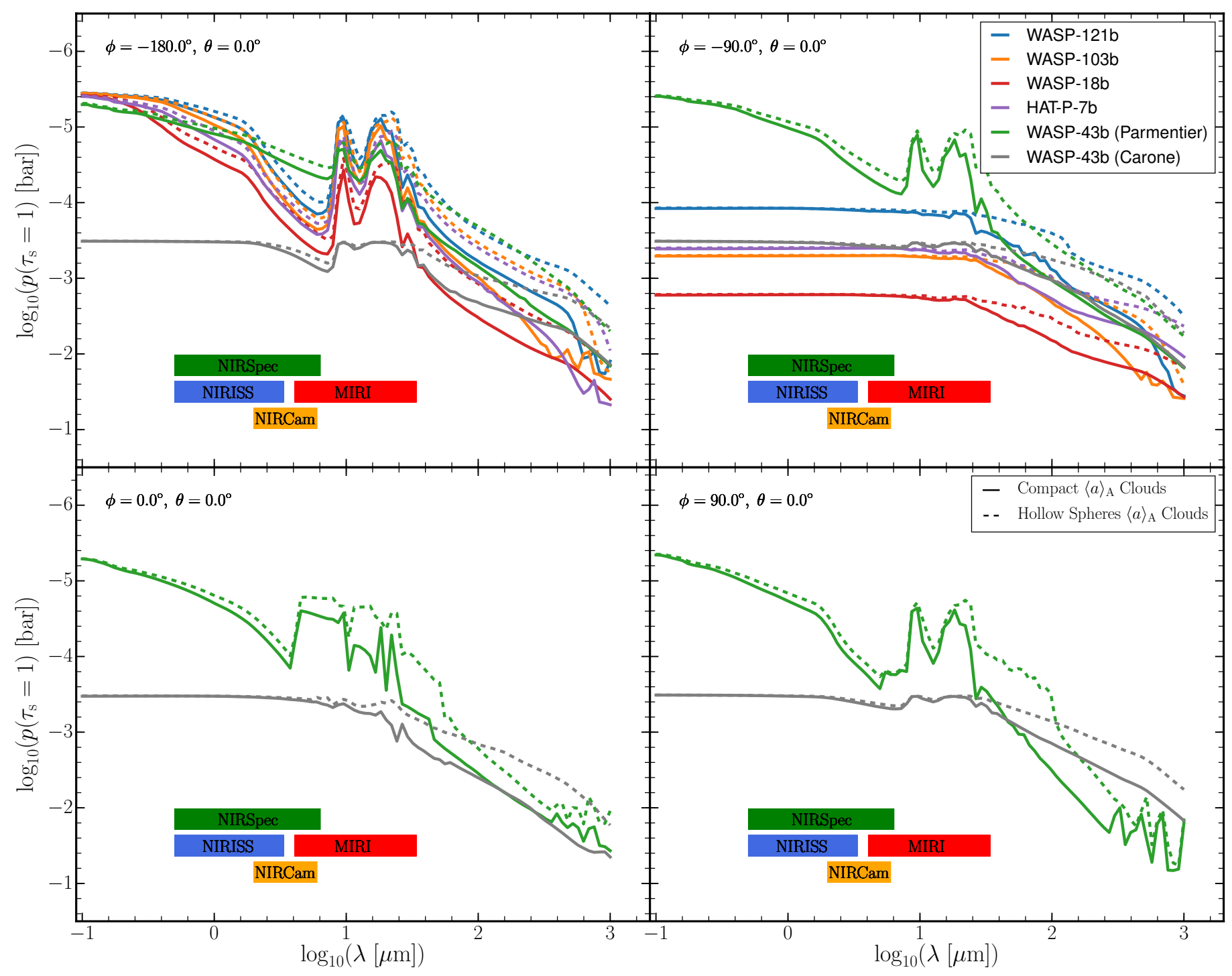

Fig. 16. Wavelength-dependent pressure level where the giant gas planets WASP-43, WASP-103b, WASP-121b, HAT-P-7b, and WASP-18b become optically thick due to cloud particles of different sizes and mixed materials forming inside these atmospheres, that is where $p_{\text {gas }}=p\left(\tau_{\mathrm{s}}(\lambda)=1\right)$, in slant geometry. All results are based on the Parmentier GCM ( $\left.T_{\text {gas }}, p_{\text {gas }}\right)$-structures, except for WASP-43b where we include both models (Parmentier GCM in green and Carone GCM in grey). When clouds remains optically thin such that $\tau_{\mathrm{s}}(\lambda)<1$, the pressure for the bottom of the atmosphere $\left(p \approx 10^{2.2}\right.$ bar in the Parmentier GCMs) is returned (hence the lines for the sub-stellar points where there is no cloud are outside the plotted range).

and WASP-121b, the optically thick pressure level appears wavelengths independent up to $\approx 25 \mu \mathrm{m}$ at the morning terminator. Whilst the optically thick pressure is constant at short wavelengths for each planet, the specific pressure varies between them from $10^{-4} \ldots 10^{-3} \mathrm{bar}$, due to the different geometric extensions. At the morning terminator $\left((\phi, \theta)=\left(-90.0^{\circ}, 0.0^{\circ}\right)\right.$, top right in Figs. 16), $p\left(\tau_{\mathrm{s}}(\lambda)=1\right)$ differs considerably between the hot gas giants and the ultra-hot Jupiters at $\lambda<30 \mathrm{~m}$. Beyond this, all planets show a trend of increasing transparency of clouds at longer wavelengths (that is increasing pressure at which $\tau_{\mathrm{s}}=1$ ), although the slope of this trend is affected by the details of the cloud micro-physics (see Sect 7.3). For the anti-stellar points $\left((\phi, \theta)=\left(-180.0^{\circ}, 0.0^{\circ}\right)\right)$, all ultra-hot Jupiters show a consistent increase in pressure where $\tau_{\mathrm{s}}(\lambda)=1$ up to between $4-6 \mu \mathrm{m}$. Hence, the atmospheric gas will be observable to greater depth and higher temperatures at these wavelengths. Strong silicate resonant features at $10 \mu \mathrm{m}$ and $20 \mu \mathrm{m}$ are prominent for all planets at the nightside, compared with only for WASP-43b at the morning terminator. Figure 16 represent the maximum atmospheric depth that can be probed remotely. Additional gas opacity may cut the observable atmosphere to lower pressure levels than those depicted.

The distinct opacity difference between the morning and the evening terminator of ultra-hot Jupiters may be probed by distinct asymmetries of the ingress and the egress in transmission light curves. Such an ingress/egress asymmetry effect due to clouds should be wavelength independent up to $\lambda \approx 20 \mu \mathrm{m}$ according to Fig. 16. An observational signature of the evening terminator would furthermore be a clear atmosphere such that the molecules in the atmosphere could be easily observable, and in the morning terminator one would see a much subdued spectral signature of gas phase molecules. The large difference in temperature between the two terminators will also play a role and will determine which gas species are present. 


\subsection{The case of WASP-43b}

WASP-43b stands out amongst the selected planets as, in comparison to the ultra-hot Jupiter results, clouds form around the entire equator, thus observations both in transmission and emission will be significantly affected by cloud. It is worth noting that the sub-stellar point is not the hottest point on WASP-43b, due to the super-rotating equatorial jet, this is located at about $\phi=30^{\circ}$ (Fig. 2 in Helling et al. 2020a). For all profiles WASP-43b shows significant silicate spectral features at $\sim 5-25 \mu \mathrm{m}$ wavelengths, although the sub-stellar point displays a very different feature shape in this spectral region. The morning and evening terminators for WASP-43b, in contrast to the ultra-hot Jupiters, are both virtually identical to the anti-stellar point, with strong silicate features and a marginally lower pressure for optically thick clouds at all wavelengths. This provides a key difference for hot giant gas planets vs ultra-hot Jupiters with total cloud cover of the terminators vs patchy cloud cover of only the morning terminator. A further notable difference for the sub-stellar point, is the total lack of $\mathrm{Fe}_{2} \mathrm{SiO}_{4}$ [s], which may explain the differences in spectral features. For the sub-stellar point, using vertically integrated optical depth (Fig. A.9) results in almost none or very weak silicate features, and look much more like the morning terminator points for the ultra-hot Jupiters.

We previously investigated the optical depth of aerosols in WASP-43b in Helling et al. (2020a), for which the compact, vertically integrated clouds are identical. However, in Helling et al. (2020a) we calculated theolin haze optical depths, we found that haze would be optically thin in the atmosphere of WASP-43b. Regardless of if compact or non-spherical shapes were considered for the haze, the clouds dominate the aerosol opacity.

WASP-43b is also a key target with JWST, full phase curve observations are planned with MIRI as part of the community early release science (Program No. 1366; PI: N. Batalha and Co-PIs: J. Bean, K. Stevenson; Bean et al. 2018), followed by a NIRspec phase curve as part of GTO Program 1224 (PI: S. Birkmann). Previous observations of WASP-43b include full phase curves with Spitzer at $3.6 \mu \mathrm{m}$ and $4.5 \mu \mathrm{m}$ (Stevenson et al. 2017) and Hubble/WFC3 (Stevenson et al. 2014) across $1.1 \ldots 1.7 \mu \mathrm{m}$. Both sets of observations produced low nightside emission possibly due to poor heat redistribution (Kataria et al. 2015), or disequilibrium chemistry and clouds (Mendonça et al. 2018). However Chubb et al. (2020) did not find statistical evidence for inclusion of clouds in their retrievals, further Venot et al. (2020a) point out differentiating cloud scenarios is difficult using Hubble/WFC3 data. Venot et al. (2020a) included cloud microphysics in some of their modelling, where they assume that magnesium silicates are composed of $\mathrm{Mg}_{2} \mathrm{SiO}_{4}$ [s] (forsterite) over $\mathrm{MgSiO}_{3}[\mathrm{~s}]$ (enstatite) and in addition including $\mathrm{Fe}[\mathrm{s}], \mathrm{Cr}[\mathrm{s}], \mathrm{MnS}[\mathrm{s}]$, and $\mathrm{Na}_{2} \mathrm{~S}[\mathrm{~s}]$. Overall they find that dayside magnesium silicate cloud species would be cold-trapped below 100 bar, and nightside cloud opacity dominated by $\mathrm{MnS}[\mathrm{s}]$ and $\mathrm{Na}_{2} \mathrm{~S}[\mathrm{~s}]$ shortward of $7 \mu \mathrm{m}$ and forsterite at longer wavelengths. However we find that clouds should be optically thick in the near-infrared across the planet at pressures as low as 0.1 mbar. At Spitzer wavelengths we only see minor differences between the profiles around the equator. As we do not include $\mathrm{MnS}$ or $\mathrm{Na}_{2} \mathrm{~S}$ comparison of models is difficult, but NIRSpec phase curve observations will provide details requiring consistent cloud chemistry. In MIRI observations, Fig. 16 shows that (using the Parmentier model) we expect to see silicate features consistent around the equator of the planet, although variable cloud abundance with latitude would still affect the strength of these features in phase curve observations, particularly for dayside emission.

Every attempt to derive spectral information from models will depend on the computational domain for which the models are simulated. Utilising the two different GCM solution for WASP-43b we show the effect on the pressure level where $\tau_{\mathrm{s}}(\lambda)=1$ for completeness. Shifting the inner boundary does affect the location and the extension of the cloud layer resulting in some lack of features to be explained by material composition of cloud, such as the sub-stellar point. The two models occupy different pressure domains: TOA(Parmentier) less than $10^{-5}$ bar, TOA Carone only to $10^{-4}$ bar. The clouds based for the Carone GCM results have larger particles sizes in their upper cloud due the higher density supporting a higher surface growth efficiency, which flatten the wavelength-dependence of the optical depth substantially. The morning terminator is comparable to that of the ultra-hot Jupiter in our sample, but for a different reason: The large particle sizes in UHJs are cause by the low nucleation rate due to their locally higher gas temperatures.

\subsection{Effects of non-sphericity}

The effects of non-spherical cloud particles are captured by a Distribution of Hollow Spheres (DHS) (Sect. 7) and are not meant to be physical interpretations of the cloud particles, but instead by averaging over a distribution of these particles, the spectroscopic effects of a distribution of irregularly shaped cloud particles are well represented. The effects of non-sphericity are surprisingly limited for the case of ultra-hot Jupiters. In Samra et al. (2020), we found that the wavelength at which clouds were no longer optically thick was increased with a DHS, however in these atmospheres (where they form) the clouds are never transparent at any wavelength. For profiles with strong silicate features, effects are largely limited to less than half an order of magnitude higher optically thick pressure levels for wavelengths longer than the silicate features, well outside the scope of what will be observable with JWST.

For profiles with subdued features (i.e. WASP-43b sub-stellar point) a DHS does enhance the silicate features, marginally. For short wavelengths $(0.1-1 \mu \mathrm{m})$ there is practically no difference between the spherical and DHS case for all profiles and planets. For profiles where the optically thick level is flat to $25 \mu \mathrm{m}$ (e.g. morning terminators for HAT-P-7b and WASP-103b) the DHS increases the optical depth for all longer wavelengths, where compact particles become increasingly transparent. However, for all the ultra-hot jupiter morning terminators, in the slant geometry a DHS does not impact the optical depth for wavelengths observable by JWST, at these wavelengths the cloud deck is flat regardless of the micro-physics.

\subsection{A chemically inert global height asymmetry tracer}

The changing day/night mean molecular weights (Figs. 12 and A.7), being representative of a $\mathrm{H} / \mathrm{H}_{2}$ dominated atmosphere gas in the cases studied here, respectively, leads to a $8 \ldots 10$ times more geometrically extended dayside compared to the nightside, if measured in hydrostatic pressure scale heights (Fig. B.1). The vertical extension of the 3D GCM causes a factor of 2 (Fig. B.2), which is not caused by the changing chemistry as the mean molecular weight is kept constant in these simulations. The effect of geometrical asymmetry is also present in the terminator regions. Such geometrical effects may be traced by observing a chemically inert species, which is not affected by element 


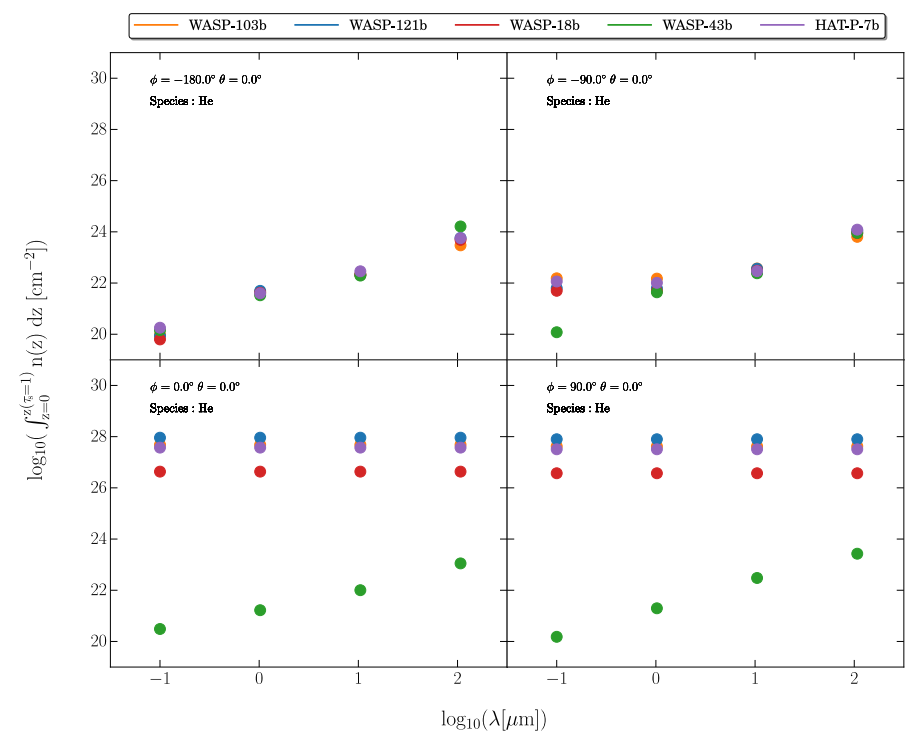

Fig. 17. Cumulative, height-integrated number density of $\mathrm{He}$, $\int_{\mathrm{z}=0}^{\mathrm{z}\left(\tau_{\mathrm{s}}=1\right)} n(z) \mathrm{d} z\left(\mathrm{~cm}^{-2}\right)$, in the optically thin region of the atmosphere $p<p\left(\tau_{\mathrm{s}}(\lambda)=1\right)$ for the sub-stellar, anti-stellar and equatorial morning and evening terminator points for $\lambda=0.1 \mu \mathrm{m}, 1.0 \mu \mathrm{m}, 10.5 \mu \mathrm{m}$ and $107 \mu \mathrm{m}$.

depletion/enrichment by cloud formation, and possibly also not by changes of the ionisation state.

Helium (He) maybe such a species and Fig. 17 shows the cumulative number density of $\mathrm{He}$ in the optically thin atmosphere (that is above the clouds) for the sub-stellar, antistellar and equatorial morning and evening terminator points at four selected wavelengths $(\lambda=0.1 \mu \mathrm{m}, 1.0 \mu \mathrm{m}, 10.5 \mu \mathrm{m}$ and $107 \mu \mathrm{m})$ to match current and future observational capabilities. CARMENES can observe at $1.0 \mu \mathrm{m}$ and JWST will be capable of observing at both $1.0 \mu \mathrm{m}$ and $10.5 \mu \mathrm{m}$. The column density of He changes with wavelength in Fig. 17 as the pressure level $p\left(\tau_{\mathrm{s}}(\lambda)=1\right)$ changes with wavelength, and hence, the geometrical extension of the optically thin atmosphere at $p<p\left(\tau_{\mathrm{s}}(\lambda)=1\right)$. The reason is the wavelength-dependent cloud opacity (see Fig. 16).

The He column density (from the top of the atmosphere to where $\left.p=p\left(\tau\left({ }_{\mathrm{s}} \lambda\right)=1\right)\right)$ is largest on the dayside and the morning terminator for the ultra-hot Jupiters as no clouds form and these values therefore represent the whole atmosphere's He column density $\left(10^{27} \ldots 10^{28} \mathrm{~cm}^{-2}\right)$. On the nightside (Fig. 17, top left), it follows the wavelength-dependent slope of $p=p\left(\tau_{\mathrm{s}}(\lambda)=1\right)$ for all sampled planets as shown in Fig. 16 (top left). The lowest He column density $\left(\approx 10^{20} \mathrm{~cm}^{-2}\right)$ occurs in the optical, the highest in the IR on the nightside $\left(\approx 10^{23} \ldots 10^{24} \mathrm{~cm}^{-2}\right)$.

\section{Discussion}

If two planets have a similar host star, similar orbital periods, radii, masses and similar undepleted element abundances, the outcome of cloud formation should be largely similar, including certainly general trends such as clouds forming on the dayside or not. Skaf et al. (2020) used HST/WCF3 data to study three (WASP-127b, WASP-79b, WASP-62b) hot gas giants that are somewhat comparable to WASP-43b. Opaque clouds were retrieved at $\approx 10^{-3}$ bar $\left(10^{2} \mathrm{~Pa}\right)$ for WASP-127b, no clouds or at $p>10^{-2}$ bar $\left(10^{3} \mathrm{~Pa}\right)$ for WASP-79b, and at $\approx 10^{-1.5}$ bar for WASP-62b, hence, at much greater depth than what our models predict. Alam et al. (2020) use HST-WCF3 data for HAT-P$32 \mathrm{Ab}$ retrieveing a (isothermal) limp temperature of $\approx 1200 \mathrm{~K}$, a cloud top pressure of $\approx 10^{-1.5}$ bar and a $\mathrm{C} / \mathrm{O}=0.12$. In our modelling, WASP-43b reaches $\tau_{\mathrm{s}}(\lambda=1 \ldots 2 \mu \mathrm{m}=1)$ at $p \approx 10^{-4}$ bar at the terminators with $T_{\text {gas }} \approx 600 \ldots 1000 \mathrm{~K}$, and with $\langle a\rangle_{\mathrm{A}} \approx$ $10^{-2} \mu \mathrm{m}$ but $\langle a\rangle_{\mathrm{A}} \approx 10^{-1} \mu \mathrm{m}$ at the dayside at the same pressure level (Fig. 6). The particles are made of a mix of metal oxides and silicates. The retrieved mean molecular weights are $2.34,2.38$, and 2.39, respectively. The values for WASP-127b, WASP-79b, WASP-62b, also within the unrealistically precise error bars, are consistent with an oxygen-depleted gas due to cloud formation. The values suggest a higher oxygen depletion than what is derived from our model for the comparable hot giant WASP-43b (Fig. A.7, top left panel). This may be consistent with the formation of bigger cloud particles that can fall deeper into the atmosphere and consume more material. However, a quantitative comparison can only be made if similar undepleted element abundances and a similarly complete gasphase chemistry is used. Alam et al. (2020) derive a very low $\mathrm{C} / \mathrm{O}$ ratio for HAT-P-32Ab, and a high metallicity for the host star. Unless these values are plagued by the retrieval approach, the low $\mathrm{C} / \mathrm{O}$ may point to either a carbon depletion and/or an initially high oxygen abundance as result of the planet formation and/or evolution processes.

The non-detection of TiO and VO on WASP-121b (Merritt et al. 2020) may be related to cloud formation at the evening terminator and to $\mathrm{TiO} / \mathrm{VO}$ being thermally unstable, hence, $\mathrm{Ti} / \mathrm{V}$ would be most abundant in their atomic (or ionic) form. Edwards et al. (2020) argue for hints of TiO and VO in combination with a grey cloud layer based on HST/WFC3 data for the ultra-hot Jupiter WASP-76b which has a equilibrium temperature comparable to HAT-P-7b. This would suggest that the cloudy evening terminator dominates the transmission spectrum. Gibson et al. (2020) present the VLT/UVES detection of Fe I in WASP-121b and retrieve the presence of a cloud later at $p \approx$ $0.15 \ldots 0.4$ bar with a local temperature of $3000 \mathrm{~K} \ldots 3700 \mathrm{~K}$. Such a temperature-pressure combination renders cloud formation impossible, and may suggest that a continuum opacity source is missing in the retrieval.

As pointed out in previous works such as Venot et al. (2020a), it is difficult to differentiate between a cloudy and cloud-free model by retrieving HST/WFC3 data alone. The influence on cloud modelling for retrievals of hot Jupiters HD 189733 b and HD 209458 b has been studied in detail by Barstow (2020). They find strong observational evidence that aerosols on HD 209458 b cover less than half of the terminator region, with unclear findings for HD 189733b. As demonstrated by the present work, there are differences between the morning and evening terminators, in terms of pressure-temperature structure, clouds, and chemistry. A 1D transmission retrieval assumes that the two terminator regions are identical. Works such as MacDonald et al. (2020) point out the requirements for multi-dimensional retrieval techniques, which are starting to be developed, in particular for analysis of emission spectra (see, e.g., Irwin et al. 2020).

\section{Conclusion}

Our modelling work for a sample of ultra-hot Jupiters suggests that these exoplanets have a large day/night cloud and gas-phase asymmetry which causes characteristic differences of their mean molecular weight, $\mathrm{C} / \mathrm{O}$ (and other element ratios), and degree of ionisation. Similar properties differ less for hot giant gas planets. 
In conclusion, we identify the following trends:

- In ultra-hot Jupiter atmospheres where $p<1$ mbar, the nightside is molecule dominated with a mean molecular weight of $\mu \approx 2.3$ in case of $\mathrm{H}_{2}$ dominating, whereas the dayside has a value representing an atomic gas such as $\mu \approx 1.3$ for atomic hydrogen. This is caused by the large temperature difference between the day- and nightsides of these exoplanets. The dayside is therefore highly thermally ionised, in addition to molecular hydrogen being thermally unstable;

- A larger mean molecular weight of $\mu=2.3$ on the nightside than originally assumed in GCMs may decrease the zonal wind velocity (Zhang \& Showman 2018);

- The day/night mean molecular weight differences cause a geometrically asymmetry between day- and nightsides in particular on tidally locked planets. We suggest a chemically inert specie such as He to probe this geometrical asymmetry;

- The immense atmospheric day/night temperature differences on ultra-hot Jupiters cause thermal ionisation to change substantially from the day- to the nightside. The thermal ionisation of the dayisde of $>10^{-5}$ is sufficient to argue for the presence of very extended, thermally driven electrically conducting daysides, namely an atmospheric ionosphere;

- The atmospheric ionosphere suggests electromagnetic coupling to a potential planetary magnetic field which maybe observable through auroral emission or a bow-shock as result of the magnetosphere-stellar wind interaction;

- Ultra-hot Jupiters can be expected to have cloud-free daysides and cloudy nightsides, in comparison to hot gas giants which have cloud-covered day- and nightsides;

- The detailed material composition is determined locally, but mineral silicate clouds made of $\mathrm{Mg} / \mathrm{Si} / \mathrm{Fe} / \mathrm{O}$ may dominate the outer cloud layers, and high-temperature condensates will dominate the inner, warmer cloud layers. Photochemical hazes may also be present but will not affect the optical depth significantly on ultra-hot Jupiters;

- Exoplanet clouds may extend further inwards than previously assumed due to the increased thermal stability for increasing gas pressures;

- The global atmosphere circulation results in cloud formation being more likely at the less-extended morning terminator, but not in the geometrically more extended, warmer evening terminator regions in ultra-hot Jupiters;

- Transmission spectra of ultra-hot Jupiters may be affected by cloud opacity at the morning terminator, but by atomic and/or ionic opacity sources at the evening terminator. This will affect the retrieval of mineral ratios such as $\mathrm{C} / \mathrm{O} /, \mathrm{Mg} / \mathrm{Si}$;

- The different cloud properties at the morning terminator of hot giant gas planets such as WASP-43b and ultra-hot Jupiters such as WASP-103b, WASP-121b, WASP18b, and HAT-P-7b cause their spectral features to differ characteristically.

Acknowledgements. Ch.H. and M.M. acknowledge funding from the European Union H2020-MSCA-ITN-2019 under Grant Agreement no. 860470 (CHAMELEON). D.S. acknowledges financial support from the Science and Technology Facilities Council (STFC), UK. for his PhD studentship (project reference 2093954), and O.H. acknowledges the $\mathrm{PhD}$ stipend from the University of St Andrews' Centre for Exoplanet Science. V.G. acknowledges the hospitality of the School of Physics \& Astronomy at the University of St Andrews and the summer student funding from the Royal Astronomical Society.

\section{References}

Alam, M. K., López-Morales, M., Nikolov, N., et al. 2020, AJ, 160, 51 Alexoudi, X., Mallonn, M., Keles, E., et al. 2020, A\&A, 640, A134

Asplund, M., Grevesse, N., Sauval, A. J., \& Scott, P. 2009, ARA\&A, 47, 481 Barstow, J. K. 2020, MNRAS, 497, 4183

Barth, P., Helling, C., Stüecken, E., et al. 2020, MNRAS

Baxter, C., Désert, J.-M., Parmentier, V., et al. 2020, A\&A, 639, A36

Bean, J. L., Stevenson, K. B., Batalha, N. M., et al. 2018, PASP, 130, 114402

Bohren, C. F., \& Huffman, D. R. 1983, Absorption and scattering of light by small particles (New York: Wiley)

Bruggeman, D. A. G. 1935, Ann. Phys., 416, 636

Caldas, A., Leconte, J., Selsis, F., et al. 2019, A\&A, 623, A16

Carone, L., Baeyens, R., Mollière, P., et al. 2020, MNRAS, 496, 3582

Carter, A. L., Nikolov, N., Sing, D. K., et al. 2020, MNRAS, 494, 5449

Casasayas-Barris, N., Pallé, E., Yan, F., et al. 2019, A\&A, 628, A9

Cauley, P. W., Shkolnik, E. L., Llama, J., \& Lanza, A. F. 2019, Nat. Astron., 3, 1128

Charnay, B., Bézard, B., Baudino, J. L., et al. 2018, ApJ, 854, 172

Chubb, K., Min, M., Kawashima, Y., Helling, C., \& Waldmann, I. 2020, A\&A, 639, A3

Cridland, A. J., van Dishoeck, E. F., Alessi, M., \& Pudritz, R. E. 2019, A\&A, 632, A63

Cridland, A. J., van Dishoeck, E. F., Alessi, M., \& Pudritz, R. E. 2020, A\&A, 642, A229

Crossfield, I. J. M., Dragomir, D., Cowan, N. B., et al. 2020, ApJ, 903, L7

Delrez, L., Santerne, A., Almenara, J.-M., et al. 2016, MNRAS, 458, 4025

Dobbs-Dixon, I., \& Agol, E. 2013, MNRAS, 435, 3159

Drummond, B., Mayne, N. J., Baraffe, I., et al. 2018, A\&A, 612, A105

Edwards, B., Changeat, Q., Baeyens, R., et al. 2020, AJ, 160, 8

Ehrenreich, D., Lovis, C., Allart, R., et al. 2020, Nature, 580, 597

Fortney, J. J. 2005, MNRAS, 364, 649

Fossati, L., Shulyak, D., Sreejith, A. G., et al. 2020, A\&A, 643, A131

Gibson, N. P., Merritt, S., Nugroho, S. K., et al. 2020, MNRAS, 493, 2215

Gillon, M., Triaud, A. H. M. J., Fortney, J. J., et al. 2012, A\&A, 542, A4

Gillon, M., Anderson, D. R., Collier-Cameron, A., et al. 2014, A\&A, 562, L3

Goeres, A. 1996, in 96, Hydrogen Deficient Stars, ASP Conf. Ser., eds. C. S. Jeffery, \& U. Heber, 69

Hellier, C., Anderson, D. R., Collier-Cameron, A., et al. 2009, Nature, 460, 1098

Helling, C. 2019, Ann. Rev. Earth Planet. Sci., 47, 583

Helling, C., \& Fomins, A. 2013, Phil. Trans. Roy. Soc. London Ser. A, 371, 10581

Helling, C., Woitke, P., Rimmer, P. B., et al. 2014, Life, 4, 142

Helling, C., Lee, G., Dobbs-Dixon, I., et al. 2016, MNRAS, 460, 855

Helling, C., Gourbin, P., Woitke, P., \& Parmentier, V. 2019a, A\&A, 626, A133

Helling, C., Iro, N., Corrales, L., et al. 2019b, A\&A, 631, A79

Helling, C., Kawashima, Y., Graham, V., et al. 2020, A\&A, 641, A178

Heng, K. 2019, MNRAS, 490, 3378

Hoeijmakers, H. J., Ehrenreich, D., Heng, K., et al. 2018, Nature, 560, 453

Hoeijmakers, H. J., Seidel, J. V., Pino, L., et al. 2020, A\&A, 641, A123

Irwin, P. G. J., Parmentier, V., Taylor, J., et al. 2020, MNRAS, 493, 106

Kataria, T., Showman, A. P., Fortney, J. J., et al. 2015, ApJ, 801, 86

Kempton, E. M. R., Bean, J. L., \& Parmentier, V. 2017, ApJ, 845, L20

Komacek, T. D., \& Showman, A. P. 2016, ApJ, 821, 16

Komacek, T. D., Showman, A. P., \& Tan, X. 2017, ApJ, 835, 198

Koskinen, T. T., Yelle, R. V., Lavvas, P., \& Y-K. Cho, J. 2014, ApJ, 796, 16

Lacy, B. I., \& Burrows, A. S. 2020, A\&A, 641, A123

Lai, D., Helling, C., \& van den Heuvel, E. P. J. 2010, ApJ, 721, 923

Lee, G., Helling, C., Dobbs-Dixon, I., \& Juncher, D. 2015a, A\&A, 580, A12

Lee, G., Helling, C., Giles, H., \& Bromley, S. T. 2015b, A\&A, 575, A11

Lee, G., Dobbs-Dixon, I., Helling, C., Bognar, K., \& Woitke, P. 2016, A\&A, 594, A48

Lendl, M., Csizmadia, S., Deline, A., et al. 2020, A\&A, 643, A94

Line, M. R., \& Parmentier, V. 2016, ApJ, 820, 78

Lines, S., Mayne, N. J., Boutle, I. A., et al. 2018, A\&A, 615, A97

Lines, S., Mayne, N. J., Manners, J., et al. 2019, MNRAS, 488, 1332

MacDonald, R. J., Goyal, J. M., \& Lewis, N. K. 2020, ApJ, 893, L43

Mansfield, M., Bean, J. L., Line, M. R., et al. 2018, AJ, 156, 10

Mendonça, J. M., Malik, M., Demory, B.-O., \& Heng, K. 2018, AJ, 155, 150

Merritt, S. R., Gibson, N. P., Nugroho, S. K., et al. 2020, A\&A, 636, A117

Mie, G. 1908, Ann. Phys., 330, 377

Min, M. 2015, in Eur. Phys. J. Web Conf., 102, 00005

Min, M., Hovenier, J. W., \& de Koter, A. 2003, A\&A, 404, 35

Min, M., Hovenier, J. W., \& de Koter, A. 2005, A\&A, 432, 909 
Min, M., Hovenier, J. W., Waters, L. B. F. M., \& de Koter, A. 2008, A\&A, 489, 135

Min, M., Ormel, C. W., Chubb, K., Helling, C., \& Kawashima, Y. 2020, A\&A, 642, A28

Molaverdikhani, K., Helling, C., Lew, B. W. P., et al. 2020, A\&A, 635, A31

Mollière, P., van Boekel, R., Dullemond, C., Henning, T., \& Mordasini, C. 2015, ApJ, 813, 47

Mollière, P., van Boekel, R., Bouwman, J., et al. 2017, A\&A, 600, A10

Mollière, P., Wardenier, J. P., van Boekel, R., et al. 2019, A\&A, 627, A67

Nichols, J. D., Burleigh, M. R., Casewell, S. L., et al. 2012, ApJ, 760, 59

Nikolov, N., Sing, D. K., Fortney, J. J., et al. 2018, Nature, 557, 526

Ormel, C. W., \& Min, M. 2019, A\&A, 622, A121

Pál, A. 2009, PhD thesis, Department of Astronomy, Eötvös Loránd University

Palik, E. D. 1985, Handbook of optical constants of solids (Academic Press)

Parmentier, V., Line, M. R., Bean, J. L., et al. 2018, A\&A, 617, A110

Parmentier, V., Showman, A. P., \& Fortney, J. J. 2021, MNRAS, 501, 78

Pearson, K. A. 2019, AJ, 158, 243

Perez-Becker, D., \& Showman, A. P. 2013, ApJ, 776, 134

Pluriel, W., Zingales, T., Leconte, J., \& Parmentier, V. 2020, A\&A, 636, A66

Powell, D., Zhang, X., Gao, P., \& Parmentier, V. 2018, ApJ, 860, 18

Rimmer, P. B., \& Helling, C. 2013, ApJ, 774, 108

Rimmer, P. B., Helling, C., \& Bilger, C. 2014, Int. J. Astrobiol., 13, 173

Rodríguez-Barrera, M. I., Helling, C., Stark, C. R., \& Rice, A. M. 2015, MNRAS, 454, 3977

Rogers, T. M. 2017, Nat. Astron., 1, 0131

Roman, M. T., Kempton, E. M. R., Rauscher, E., et al. 2021, ApJ, 908, 101

Salz, M., Czesla, S., Schneider, P. C., et al. 2018, A\&A, 620, A97

Samra, D., Helling, C., \& Min, M. 2020, A\&A, 639, A107

Selhorst, C. L., Barbosa, C. L., Simões, P. J. A., Vidotto, A. A., \& Valio, A. 2020, ApJ, 895, 62
Sheppard, K. B., Mandell, A. M., Tamburo, P., et al. 2017, ApJ, 850, L32 Showman, A. P., Fortney, J. J., Lian, Y., et al. 2009, ApJ, 699, 564

Showman, A. P., Tan, X., \& Parmentier, V. 2020, Space Sci. Rev., 216, 139

Sing, D. K., Fortney, J. J., Nikolov, N., et al. 2016, Nature, 529, 59

Sing, D. K., Lavvas, P., Ballester, G. E., et al. 2019, AJ, 158, 91

Skaf, N., Bieger, M. F., Edwards, B., et al. 2020, AJ, 160, 109

Stevenson, K. B., Désert, J.-M., Line, M. R., et al. 2014, Science, 346, 838

Stevenson, K. B., Line, M. R., Bean, J. L., et al. 2017, AJ, 153, 68

Stolker, T., Min, M., Stam, D. M., et al. 2017, A\&A, 607, A42

Teske, J., Díaz, M. R., Luque, R., et al. 2020, AJ, 160, 96

Thorngren, D., Gao, P., \& Fortney, J. J. 2019, ApJ, 884, L6

Torres, G., Winn, J. N., \& Holman, M. J. 2008, ApJ, 677, 1324

Turner, J. D., de Mooij, E. J. W., Jayawardhana, R., et al. 2020, ApJ, 888, L13

Van Eylen, V., Kjeldsen, H., Christensen-Dalsgaard, J., \& Aerts, C. 2012, Astron. Nachr., 333, 1088

Varela, J., Réville, V., Brun, A. S., Zarka, P., \& Pantellini, F. 2018, A\&A, 616, A182

Venot, O., Parmentier, V., Blecic, J., et al. 2020, ApJ, 890, 176

Vidotto, A. A., Jardine, M., \& Helling, C. 2010, ApJ, 722, L168

Vidotto, A. A., Jardine, M., \& Helling, C. 2011, MNRAS, 411, L46

Wang, H., \& Wordsworth, R. 2020, ApJ, 891, 7

Woitke, P., \& Helling, C. 2004, A\&A, 414, 335

Woitke, P., Helling, C., Hunter, G. H., et al. 2018, A\&A, 614, A1

Wyttenbach, A., Mollière, P., Ehrenreich, D., et al. 2020, A\&A, 638, A87

Yan, F., \& Henning, T. 2018, Nat. Astron., 2, 714

Yan, F., Pallé, E., Reiners, A., et al. 2020, A\&A, 640, L5

Zaghoo, M., \& Collins, G. W. 2018, ApJ, 862, 19

Zhang, X. 2020, Res. Astron. Astrophys., 20, 099

Zhang, X., \& Showman, A. P. 2017, ApJ, 836, 73

Zhang, X., \& Showman, A. P. 2018, ApJ, 866, 2 


\section{Appendix A: Cloud properties and gas-phase parameters $\left(\mathrm{C} / \mathrm{O}, \mu, f_{\mathrm{e}}\right)$}

In order to enable model comparability, we provide here the detailed results of the cloud complex for the ultra-hot Jupiters which form the base for the more condensed representation within the main text body. Figure A.1 provides all the dustto- gas ratio, $\rho_{\mathrm{d}} / \rho$, profiles clearly indicating where in these atmosphere most of the cloud particle mass is located according to our model. Figures A.2-A.5 provide the details of the 16 considered bulk growth materials as part of our kinetic cloud formation model for the equatorial nightside and the morning terminator only. Mineral clouds do not form on the dayside and the evening terminator due to unfavourably high local gas temperatures. Panels appear empty where no cloud particle formation occurs; this results from profiles where no nucleation seeds form. Figure A.6-A.8 provide the detailed results of the carbon-to-oxygen ratio $(\mathrm{C} / \mathrm{O})$, the mean molecular weight, $\mu$, and the thermal degree of ionisation, $f_{\mathrm{e}}$ as data input for the averaged values shown in previous sections.

Figure A.9 provides the individual plots for the pressure where optical depth of unity (both vertical and slant geometry) is reached for the five sample planets, the hot giant gas planets such as WASP-43b and ultra-hot Jupiters such as WASP-103b, WASP-121b, WASP18b, and HAT-P-7b.
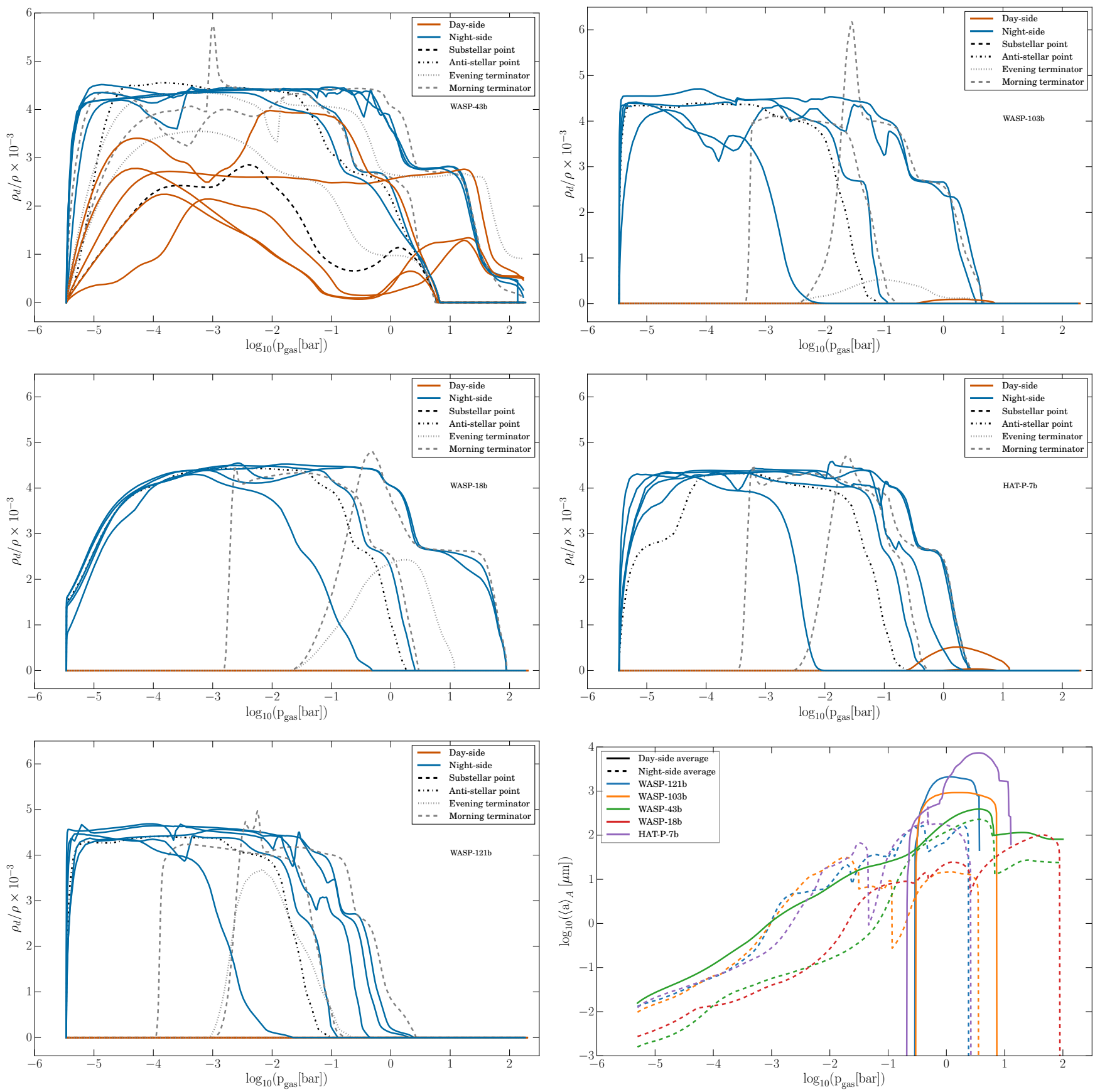

Fig. A.1. Dust-to-gas ratio, $\rho_{\mathrm{d}} / \rho$, for the giant gas planet WASP-43b, and the ultra-hot Jupiters WASP-18b, HAT-P-7b, WASP-103b, and WASP-121b. 

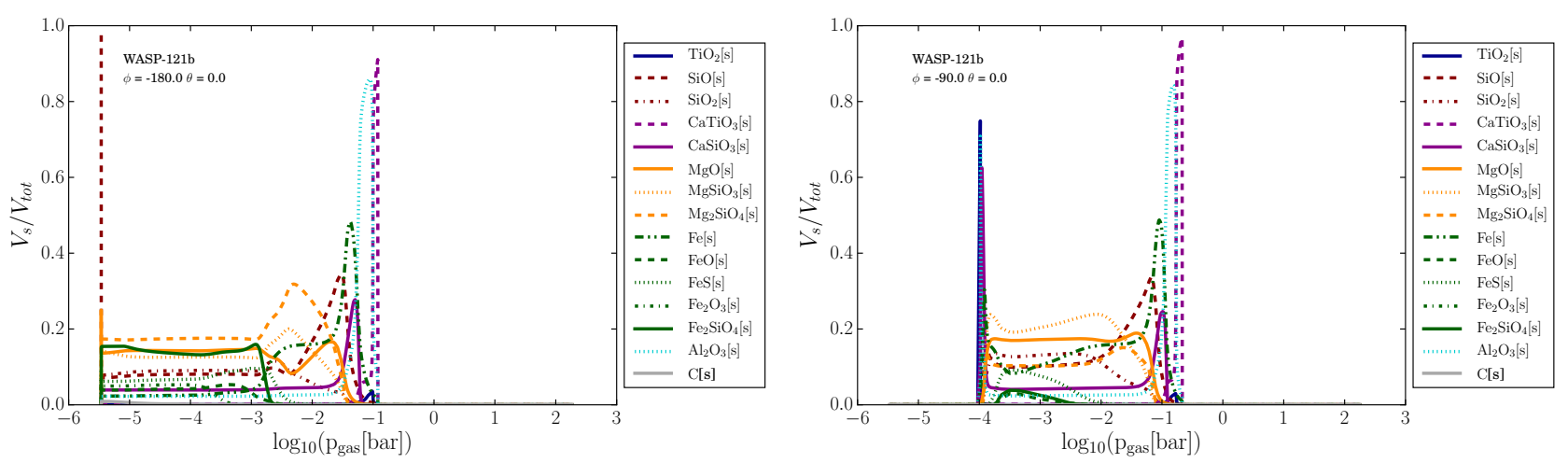

Fig. A.2. Individual bulk material volume fractions WASP-121b. Anti-stellar point: $(\phi, \theta)=\left(-180.0^{\circ}, 0.0^{\circ}\right)$ and equatorial morning terminator: $(\phi, \theta)=\left(-90.0^{\circ}, 0.0^{\circ}\right)$.
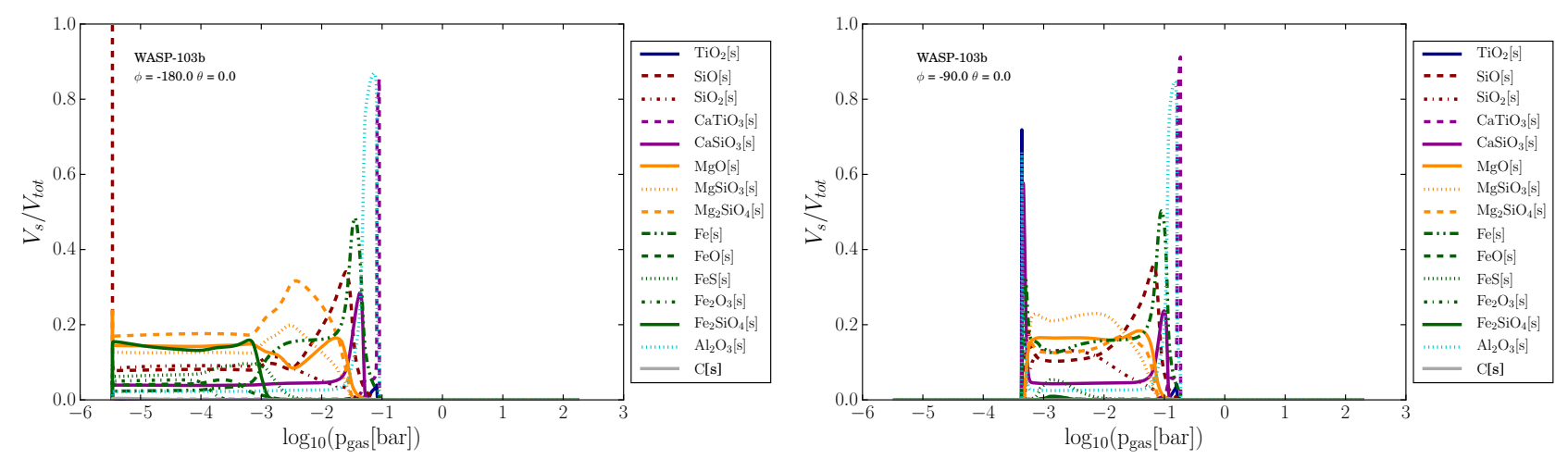

Fig. A.3. Individual bulk material volume fractions WASP-103b. Anti-stellar point: $(\phi, \theta)=\left(-180.0^{\circ}, 0.0^{\circ}\right)$ and equatorial morning terminator: $(\phi, \theta)=\left(-90.0^{\circ}, 0.0^{\circ}\right)$.
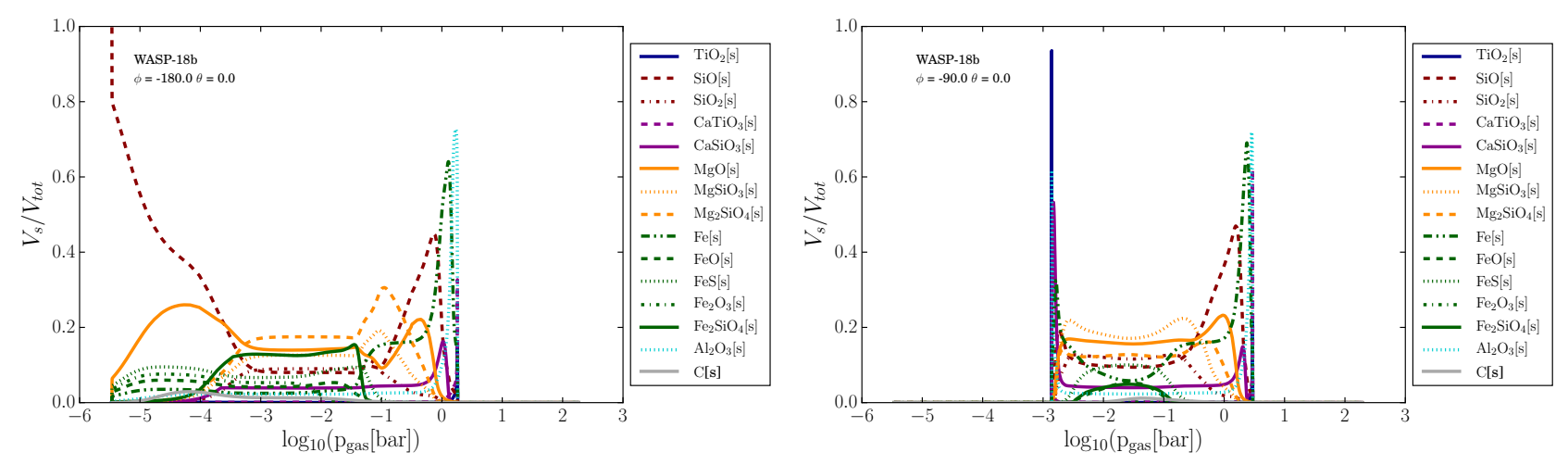

Fig. A.4. Individual bulk material volume fractions WASP-18b. Anti-stellar point: $(\phi, \theta)=\left(-180.0^{\circ}, 0.0^{\circ}\right)$ and equatorial morning terminator: $(\phi, \theta)=\left(-90.0^{\circ}, 0.0^{\circ}\right)$.
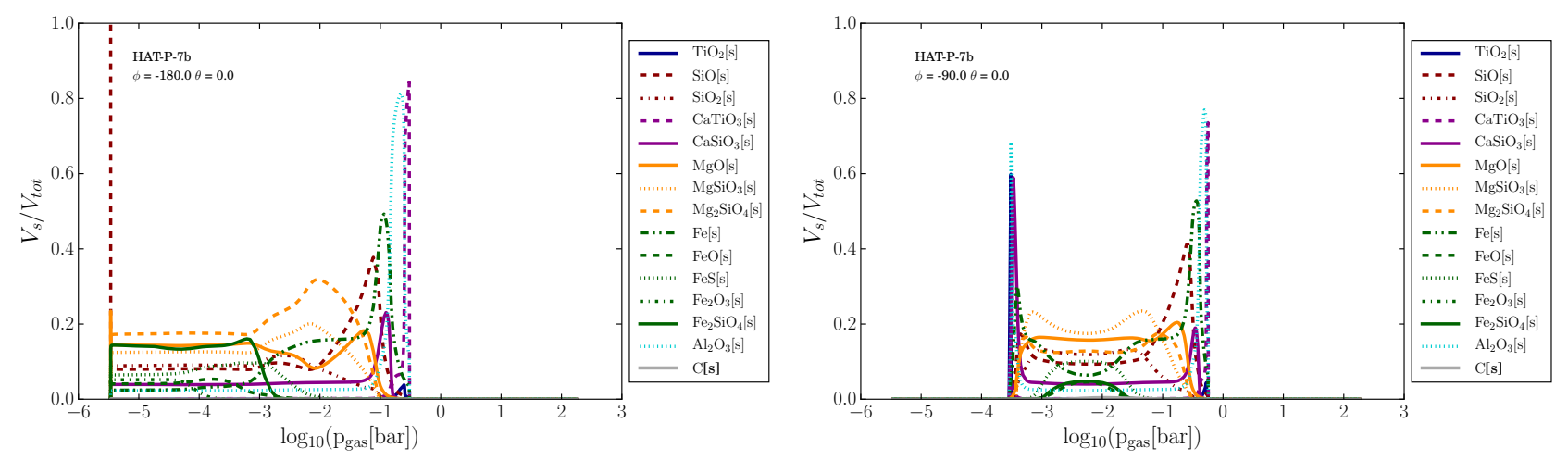

Fig. A.5. Individual bulk material volume fractions HAT-P-7b. Anti-stellar point: $(\phi, \theta)=\left(-180.0^{\circ}, 0.0^{\circ}\right)$ and equatorial morning terminator: $\left.(\phi, \theta)=\left(-90.0^{\circ}, 0.0^{\circ}\right).\right)$ 
Ch. Helling et al.: Trending clouds in hot giant gas planets and ultra-hot Jupiters
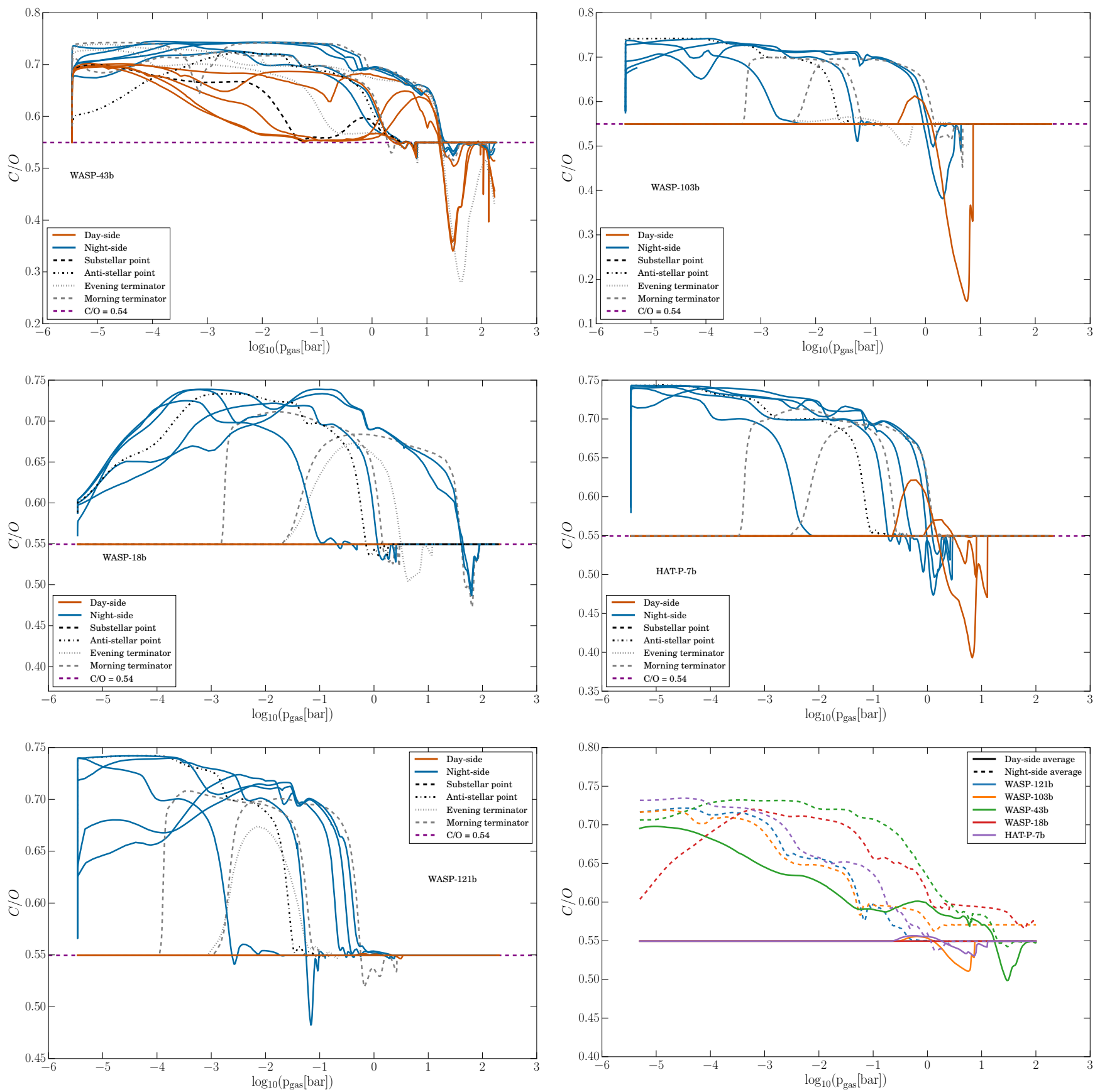

Fig. A.6. Carbon-to-oxygen ratio (C/O) for the giant gas planet WASP-43b, and the ultra-hot Jupiters WASP-18b, HAT-P-7b, WASP-103b, and WASP-121b. The solar value $\mathrm{C} / \mathrm{O}=0.54$ is plotted in dashed purple. 
A\&A 649, A44 (2021)
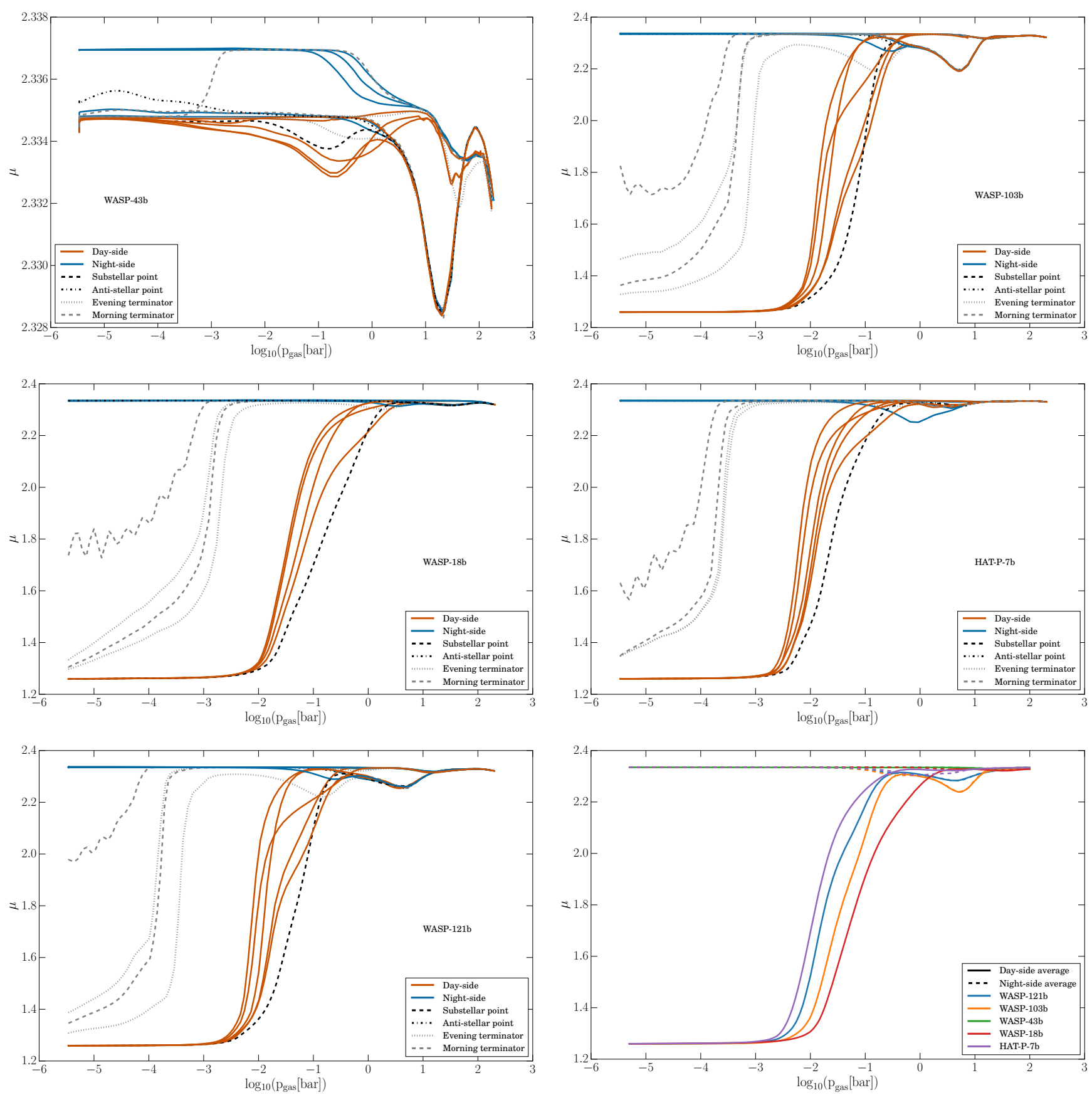

Fig. A.7. Atmospheric mean molecular weight, $\mu$, for the giant gas planet WASP-43b, and the ultra-hot Jupiters WASP-18b, HAT-P-7b, WASP$103 \mathrm{~b}$, and WASP-121b. The ultra-hot Jupiters show significant differences in $\mu$ between the dayside and nightside of the planets, whereas WASP-43b shows an approximately constant value around $\mu \approx 2.3$. 
Ch. Helling et al.: Trending clouds in hot giant gas planets and ultra-hot Jupiters
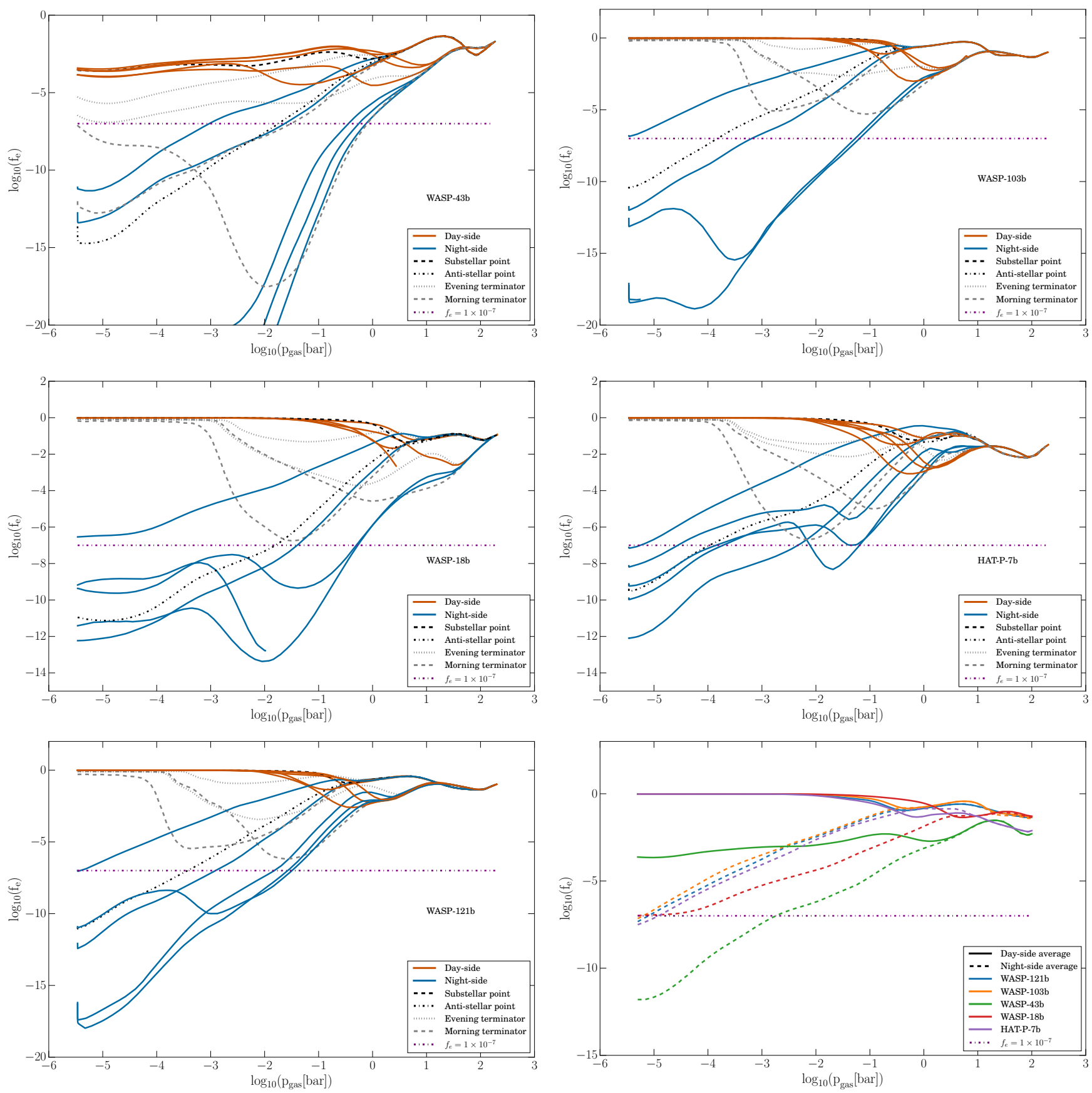

Fig. A.8. Degree of thermal ionisation, $f_{\mathrm{e}}=p_{\mathrm{e}} /\left(p_{\text {gas }}+p_{\mathrm{e}}\right)$. The dash-dot purple line shows $f_{\mathrm{e}}=1 \times 10^{-7}$ as a threshold for plasma behaviour. All ultra-hot Jupiters have dayside thermal ionisation $f_{\mathrm{e}}>10^{-4}$ suggesting an extended dayside ionosphere. Most of the cloud-forming nightsides are little affected by thermal ionisation. 

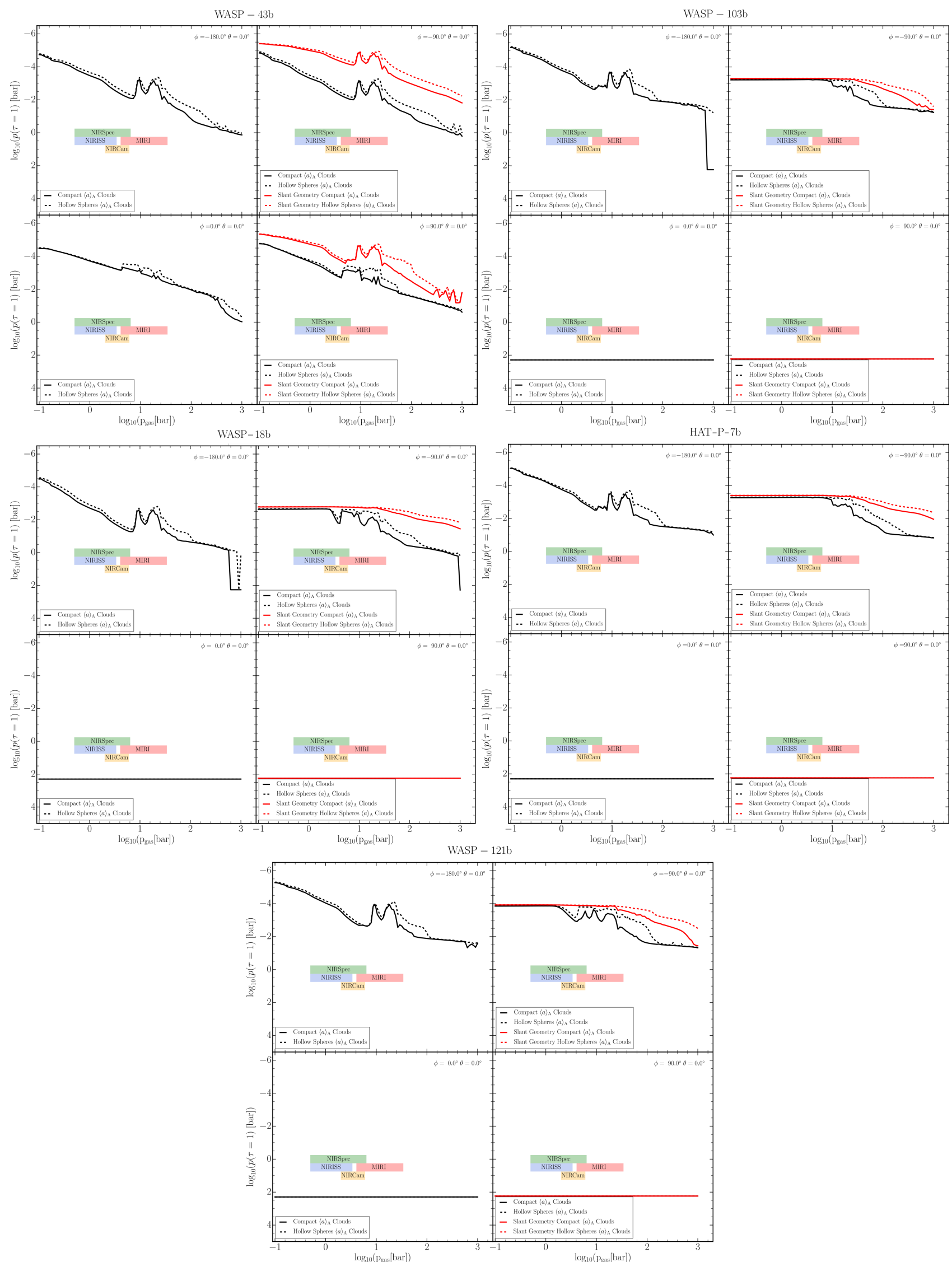

Fig. A.9. Wavelength-dependent pressure level, $p_{\text {gas }}=p(\tau=1)$ where atmospheric gas above the clouds become optically thick (where $\left.\tau=1\right)$. Included is slant geometry (red curves, using $\tau=\tau_{\mathrm{s}}$ Eq. (6)) and plane-parallel geometry (dashed) as comparison. When the optical depth of clouds never reaches 1 , the pressure for the bottom of the atmosphere (here $p \approx 10^{2.2}$ bar) is returned (hence the lines for the sub-stellar points where there is no cloud). 


\section{Appendix B: Global atmosphere height asymmetries and hydrostatic pressure scale height}
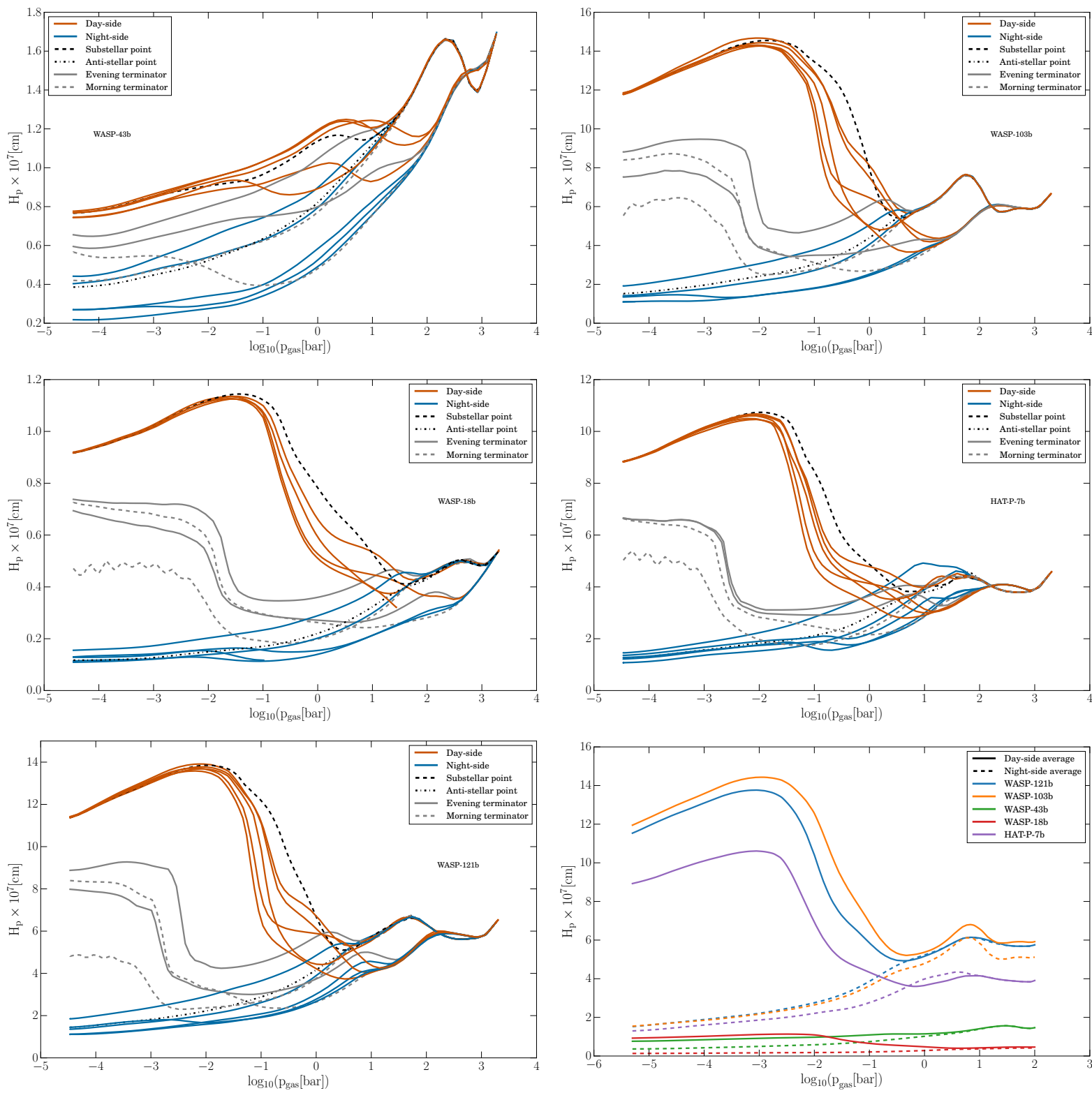

Fig. B.1. Hydrostatic pressure scale height $\left(H_{\mathrm{p}}=(\mathrm{kT})\left(\mu \mathrm{m}_{\mathrm{H}} \mathrm{g}\right)\right)$ for the giant gas WASP-43b, and the ultra-hot Jupiters HAT-P-7b, WASP-18b, and WASP-103b, WASP-121b. The changing pressure scale height is caused by the temperature-dependent mean molecular weight, $\mu(T)$, that changes from the day- to the nightside due to the large differences in gas temperatures (see Fig. A.7).

We consider the (vertical) geometrical extension of the atmosphere (Fig. B.2), and provide a comparison to the hydrostatic pressure scale height for completeness (Fig. B.1). This geometric height is of interest as it gives an indication for the asymmetry of the atmosphere. For example, have Salz et al. (2018) observe an asymmetric transit in the He I line at $0.1083 \AA$ with CARMENES in conjunction with a net blue shift of $-3.5 \pm 0.4 \mathrm{~km} \mathrm{~s}^{-1}$. One interpretation is a geometrical day/night asymmetry of $0.2 R_{\mathrm{P}}$. Figure B.2 demonstrates that the atmosphere of close-in planets are not spherical symmetric. The day/night geometric extension for the ultra-hot Jupiters in our sample is $\approx 2$, but this is not a result of the changing mean molecular weight as a constant mean molecular weight is assumed in the 3D GCMs utilised here.
Table B.1 presents the effect of the changing geometrical extension in terms of a potential transit depth $\delta_{\text {transit }}=\left(\left(R_{\mathrm{P}}+z\right) / R_{\text {star }}\right)^{2}$ $(z-$ vertical extension of the atmosphere, Fig. B.2).

The effect of the changing mean molecular weight is better seen in considering the hydostatic pressure scale height which was derived after the gas-phase chemistry was solved within our cloud formation model (Fig. B.1). Clearly visible in all plots is the onset of the thermal inversions, which produces an increase in the rate at which the vertical extent of the atmosphere grows at higher altitudes (moving left in the Fig. B.2). This change is most noticeable for the ultra-hot Jupiters, for the terminators as they have the steepest inversions. The terminator profiles initially have atmospheric extensions similar to nightside profiles 

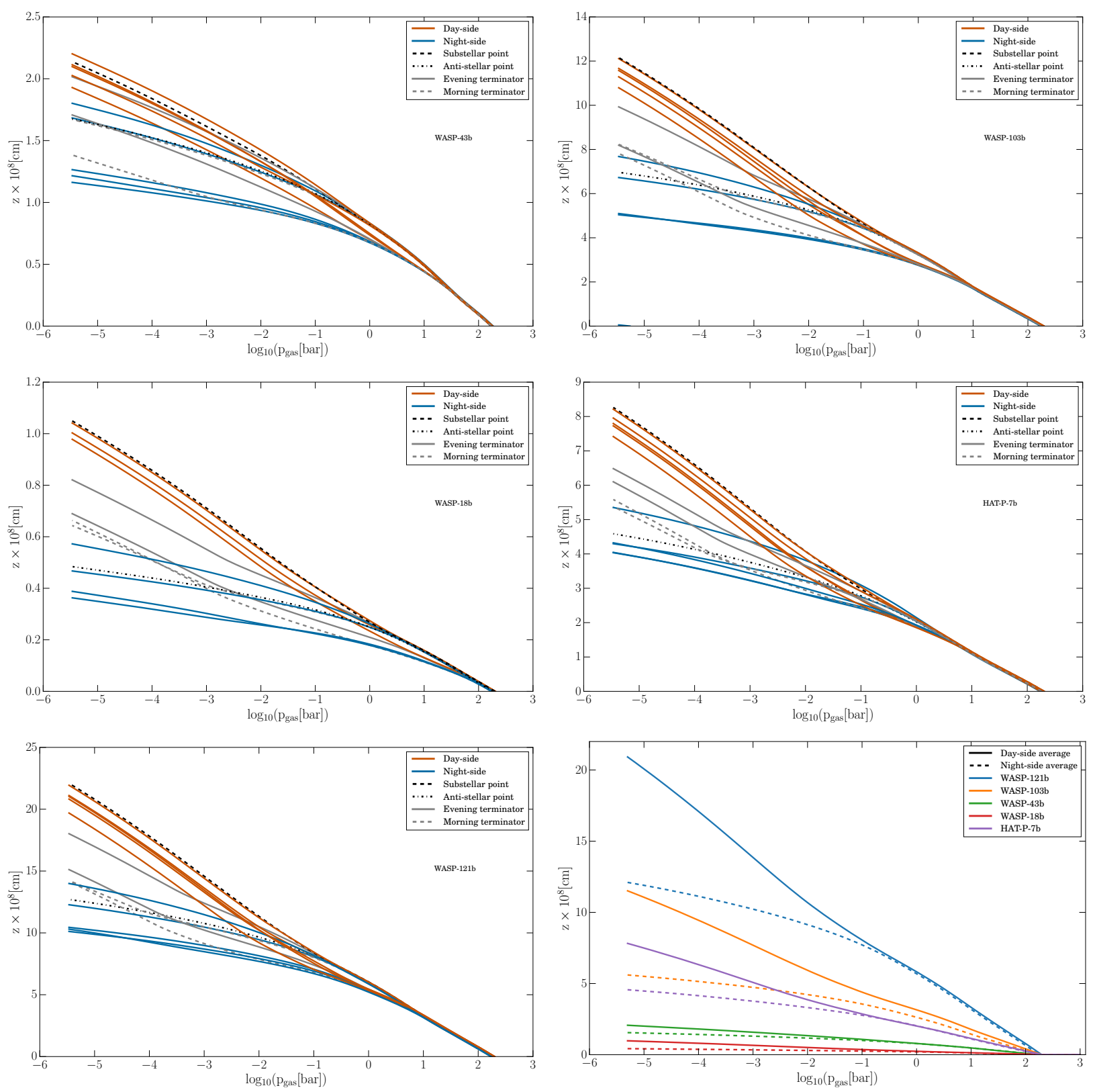

Fig. B.2. Geometric atmosphere height, $z$ [cm], calculated by summing the change in height $\mathrm{d} z$ between successive pressure layers starting from the inner boundary where $z=0$.

Table B.1. Maximum average dayside and nightside extensions in terms of planetary radius, the day-to-nightside extension ratio and the expected transit depth calculated as $\delta_{\text {transit }}=\left(\left(R_{\mathrm{P}}+z\right) / R_{\text {star }}\right)^{2}$.

\begin{tabular}{cccccc}
\hline \hline Planet & WASP-103b & WASP-18b & WASP-121b & HAT-P-7b & WASP-43b \\
\hline Average dayside maximum extension $\left(R_{\mathrm{P}}\right)$ & 0.10777 & 0.0126 & 0.16558 & 0.07815 & 0.02856 \\
Average nightside maximum extension $\left(R_{\mathrm{P}}\right)$ & 0.05244 & 0.0055 & 0.09582 & 0.04561 & 0.00214 \\
Day-/nightside ratio & 2.055 & 2.294 & 1.728 & 1.713 & 1.334 \\
$\delta_{\text {transit }}(\%)$ & 1.141 & 0.834 & 1.548 & 1.725 & 2.432 \\
\hline
\end{tabular}

Notes. We use the $R_{\mathrm{p}}$ and $R_{\text {star }}$ given in Table 1, and the vertical extension of the atmosphere $z$ (Fig. B.2).

but around the millibar level they switch to a gradient parallel to the dayside profiles. The lower right of figure shows the difference between average the dayside and nightside profiles, with the general trend that in the deep atmosphere the extension is the same, but at higher altitudes diverges, this altitude similarly corresponds with the drop in mean molecular weight (see bottom right Fig. A.7. It also shows that the effect is most prominent for planets with low surface gravities; WASP-18b shows little difference in extension despite being an ultra-hot Jupiter as it has a significantly higher surface gravity). Multiple 
Ch. Helling et al.: Trending clouds in hot giant gas planets and ultra-hot Jupiters
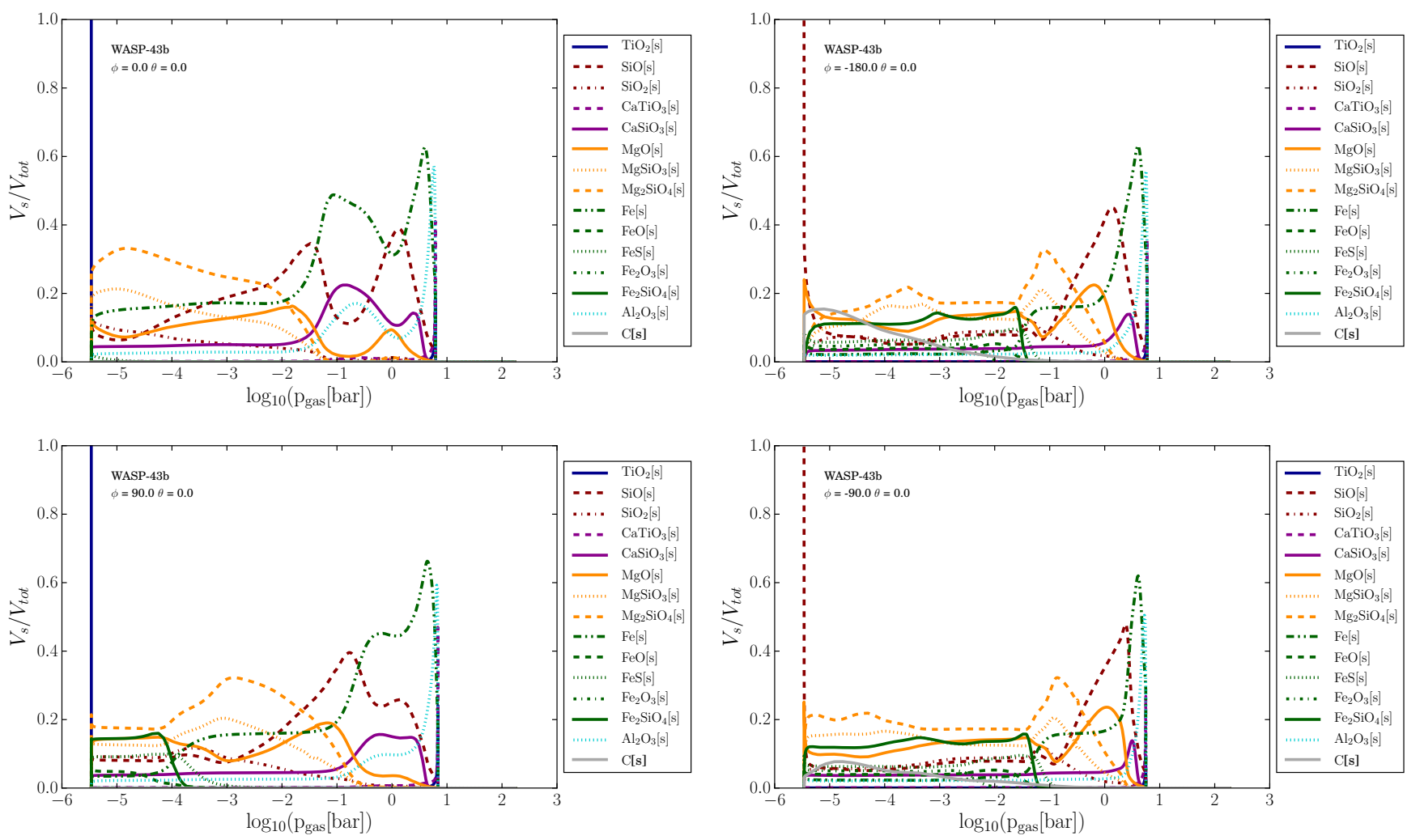

Fig. B.3. Individual bulk material volume fractions WASP-43b (based on the 1D thermodynamic profiles from Parmentier et al.). Sub-stellar point: $(\phi, \theta)=\left(0.0^{\circ}, 0.0^{\circ}\right)$, anti-stellar point: $(\phi, \theta)=\left(-180.0^{\circ}, 0.0^{\circ}\right)$, equatorial morning terminator: $(\phi, \theta)=\left(-90.0^{\circ}, 0.0^{\circ}\right)$, equatorial evening terminator: $(\phi, \theta)=\left(90.0^{\circ}, 0.0^{\circ}\right)$
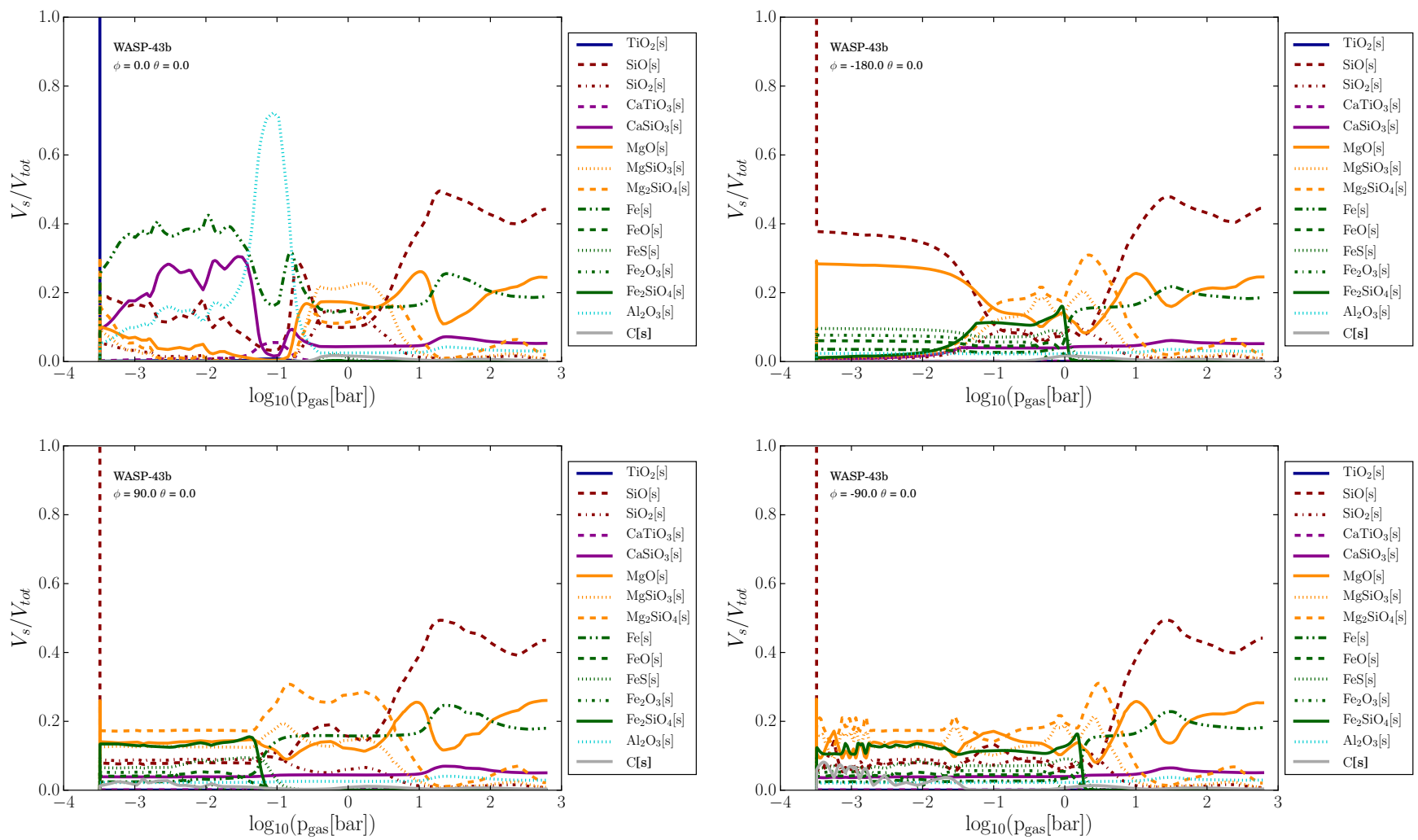

Fig. B.4. Individual bulk material volume fractions WASP-43b (based on the $1 \mathrm{D}$ thermodynamic profiles from Carone et al.). Sub-stellar point: $(\phi, \theta)=\left(0.0^{\circ}, 0.0^{\circ}\right)$, anti-stellar point: $(\phi, \theta)=\left(-180.0^{\circ}, 0.0^{\circ}\right)$, equatorial morning terminator: $(\phi, \theta)=\left(-90.0^{\circ}, 0.0^{\circ}\right)$, equatorial evening terminator: $(\phi, \theta)=\left(90.0^{\circ}, 0.0^{\circ}\right)$ 

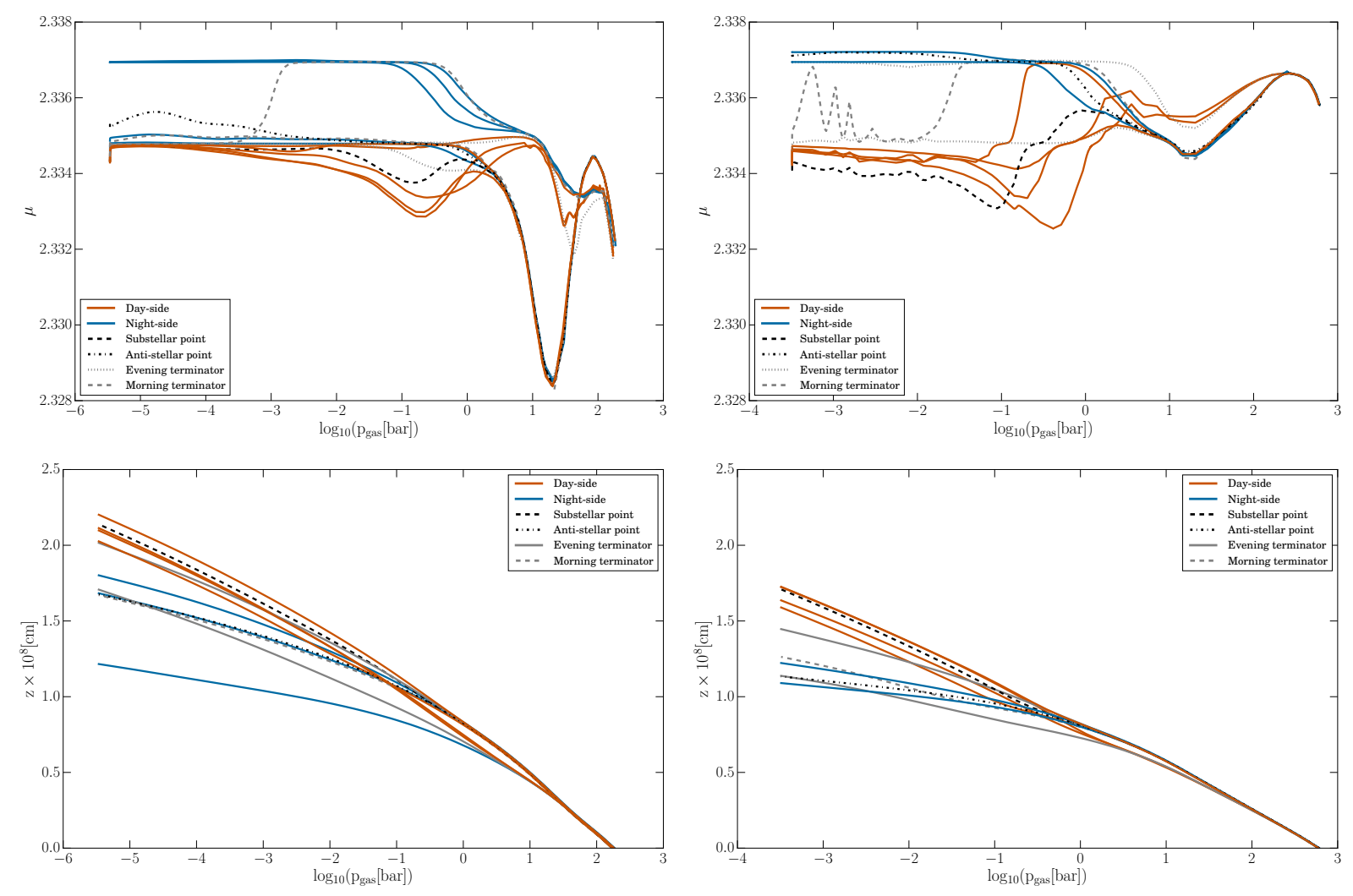

Fig. B.5. Effect of the inner boundary of the 3D GCM models on the mean molecular weight, $\mu$, and the geometric atmosphere extension for the giant gas planet example WASP-43b. Right: based on the 1D thermodynamic profiles from Parmentier et al. Right: based on the 1D thermodynamic profiles from Carone et al. There is a difference of $\sim 0.5 \times 10^{8} \mathrm{~cm}$ between the day and night geometric extensions, of the same profiles, between the two models.

studies have now investigated the effects of different extensions on the day- and nightsides in transmission spectra, (Caldas et al. 2019; Pluriel et al. 2020) found changes in temperature and compositional gradient across the terminator region can bias results of retrievals, and further that this is dependent on the gradient of the change as this affects the extent of the atmosphere and thus the amount of the dayside that the light ray passes through.

Figure B.5 provides the detailed results on the mean molecular weight, $\mu$, and geometric extension for the Parmentier/Carone in order to enable comparison with future simulations.

\section{Appendix C: Supplementary details on the WASP-43b simulations results by Parmentier and Carone}

The detailed cloud modelling results regarding the cloud material volume fractions, $V_{\mathrm{s}} / V_{\mathrm{tot}}$, and the results for the mean molecular weight, $\mu$, and the vertical, geometric extension, $z$, for the comparative study if the effect of the inner boundary for the example of WASP-32b in Sect. 6 are provided. 